Portland State University

PDXScholar

$1-1-2010$

\title{
Spousal Crossover Effects of Job Demands on Health
}

Rachel Jane Daniels

Portland State University

Follow this and additional works at: https://pdxscholar.library.pdx.edu/open_access_etds Let us know how access to this document benefits you.

\section{Recommended Citation}

Daniels, Rachel Jane, "Spousal Crossover Effects of Job Demands on Health" (2010). Dissertations and Theses. Paper 95.

https://doi.org/10.15760/etd.95

This Dissertation is brought to you for free and open access. It has been accepted for inclusion in Dissertations and Theses by an authorized administrator of PDXScholar. Please contact us if we can make this document more accessible: pdxscholar@pdx.edu. 
Spousal Crossover Effects of Job Demands on Health

\title{
by
}

Rachel Jane Daniels

A dissertation submitted in partial fulfillment of the requirements for the degree of

\author{
Doctor of Philosophy \\ in \\ Applied Psychology
}

\author{
Dissertation Committee: \\ Leslie Hammer, Chair \\ Cynthia Mohr \\ Donald Truxillo \\ Charlotte Fritz \\ Elizabeth Almer
}

Portland State University

(C) 2010 


\begin{abstract}
The primary goal of this dissertation was to examine the crossover effects of an individual's job demands on the perceived health of the spouse. Using a sample of grocery store employees and their partners, crossover relationships were examined for 90 dyads using Structural Equation Modeling. Although the models had good fit, the proposed direct crossover relationships of job demands on health were not supported. Job demands of one partner did not significantly predict health outcomes in the other partner; although follow-up analyses found significant crossover of women's job satisfaction on men's mental health scores. An additional crossover relationship was supported with the finding that crossover of health between spouses (i.e. physical and mental health components of general health) was significant. The second goal of the present dissertation study was to develop a typology of crossover research. All crossover studies to date were reviewed and categorized in a discussion of the proposed typology.
\end{abstract}




\section{Acknowledgements}

I would like to acknowledge my committee members for their constructive feedback on earlier versions of this paper: Leslie Hammer, Cynthia Mohr, Donald Truxillo, Charlotte Fritz, and Elizabeth Almer. The five years of guidance, support and tough love provided by my inspirational and hard-working advisor, Leslie Hammer, is greatly appreciated. Additional gratitude is extended to Cynthia Mohr for the extra care she took to ensure this dissertation met the highest of standards. I would also like to acknowledge my dear friends and colleagues Kristi Zimmerman and Deborah Ford Martin. The completion of my degree would not have been possible without their love, support and sage advice. 
Table of Contents

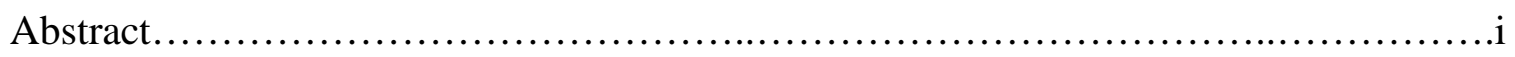

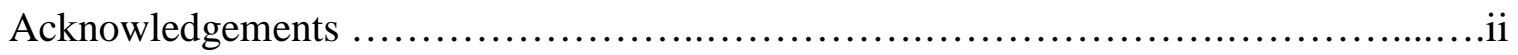

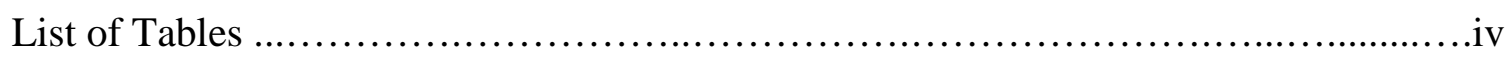

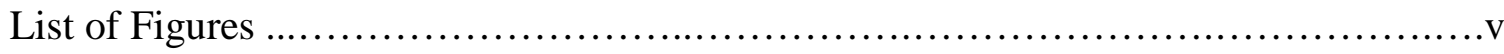

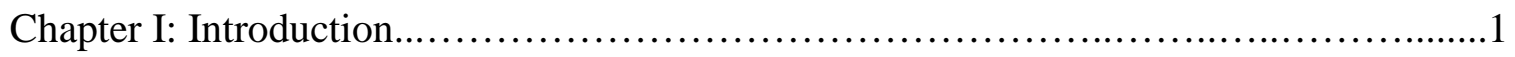

Chapter II: Crossover Effects $\ldots \ldots \ldots \ldots \ldots \ldots \ldots \ldots \ldots \ldots \ldots \ldots \ldots \ldots \ldots \ldots \ldots \ldots \ldots \ldots \ldots . .8$

Chapter III: Job Demands and Health.. ........................................ 37

Chapter IV: Health......................................................44

Chapter V: Income Adequacy as a Common Stressor ..............................53

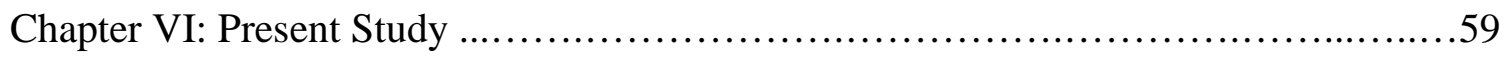

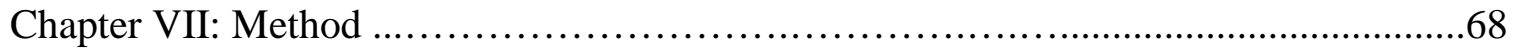

Chapter VIII: Results .....................................................79

Chapter IX: Discussion .................................................... 99

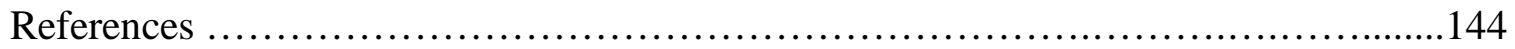

Appendix A: Crossover Research...........................................169

Appendix B: Study Measures................................................. 188 
List of Tables

Table 1: Means and SDs for APIM Demographics

Table 2: Frequencies and Percentages: APIM Demographics for Males

Table 3: Frequencies and Percentages: APIM Demographics for Females. 128

Table 4: Intercorrelations between Study Variables

Table 5: Unstandardized Effects, Standardized Effects, Correlations, and Significance

Levels for APIM in Figure $1(\mathrm{~N}=90)$

Table 6: Unstandardized Effects, Standardized Effects, Correlations, and Significance

Levels for APIM in Figure $2(\mathrm{~N}=90)$ 131

Table 7: Demographics for Post-Hoc Analyses Addressing JDCS Model

Table 8: Post-Hoc Hierarchical Multiple Regression Analyses addressing JDCS Model,

Part 1

Table 9: Post-Hoc Hierarchical Multiple Regression Analyses addressing JDCS Model,

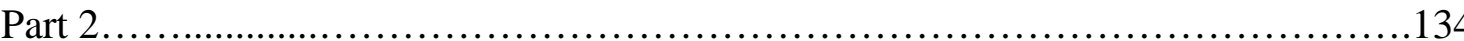

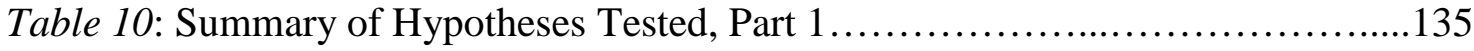

Table 11: Summary of Hypotheses Tested, Part 2.................................136 
List of Figures

Figure 1: APIM Predicting Physical Health......................................137

Figure 2: APIM Predicting Mental Health........................................... 138

Figure 3: Alternate APIM 1.................................................... 139

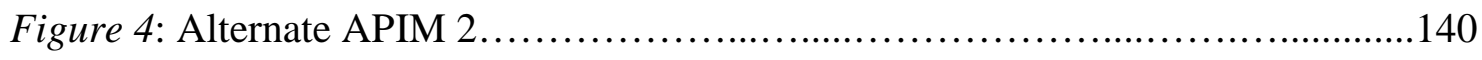

Figure 5: Alternate APIM 3 ....................................................... 141

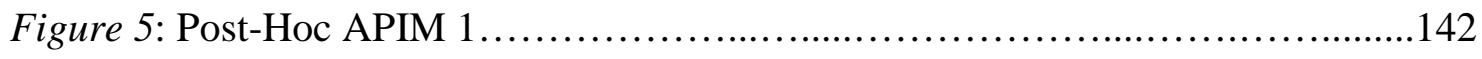

Figure 5: Post-Hoc APIM 2..................................................143 


\section{Chapter I}

\section{Introduction}

There has been a dramatic shift in family demographics to include more dualearner families and greater involvement of men in family care. Dual-earner couples comprised $78 \%$ of working couples in 2002 , while only $66 \%$ of this population was dualearner in 1997 (Bond, Thompson, Galinsky, \& Prottas, 2002). In 2004 approximately 73 million employees in the United States were in dual-earner relationships (Bureau of Labor Statistics, 2005). Not only does this group of 73 million employees have to deal with the interface between their own work and home lives, but they also must concern themselves with how their partners manage the interface between work and home. In order to present a complete portrayal of the impact stressors at work can have, researchers must look beyond the outcomes affecting the individual employees. The effects on spouses of these employees must be considered.

Research has established the connection between the work role and the family role, showing that demands in one domain spillover and affect the other domain (Frone, 2003). It has been demonstrated that the demands stemming from the work role can have a negative impact on the health of the employee (Bellavia \& Frone, 2005). More specifically, research has demonstrated that job demands are strongly linked with the health of employees (van der Doef \& Maes, 1999). According to Systems Theory, family members are interconnected. Thus, a critical next step is to examine the connection between the characteristics of one partner's job and the health of his or her family.

It is important to understand how workplace stress influences the health of the employee's spouse. Understanding the bigger picture of how work stressors affect the 
health of the employee and his or her partner can help researchers and practitioners understand the far-reaching impact that job design can have. It is important that management understand the full effects of stressful work environments. For example, job design may have an impact on individual and organizational costs associated with the employee (e.g. absenteeism, productivity, turnover). Further, negative impacts of work design may lead to costs associated with the spouse of the employee (e.g. increased family-to-work conflict, health insurance costs). It is important to explore the wide range of impacts that job design can have on the family system to make the case to management about the value of improving the work environment for employees. Further, these issues are especially relevant in low-wage or hourly populations; in which employees may not have access to resources to deal with additional stressors (Heymann, 2006).

Crossover is a process that may be used to explain how one partner's stress or strain in the workplace may influence his or her spouse in the home domain. According to Bolger, DeLongis, Kessler and Worthington (1989), crossover is a dyadic, interindividual transmission of stress or strain, occurring when stress experienced in the workplace by the individual leads to stress experienced at home by the individual's spouse. As will be discussed in the next chapter, there are three main mechanisms that lead to crossover within a dyad (Westman \& Vinokur, 1998). The present study addressed two of these mechanisms: direct crossover and common stressors crossover.

Although there have been 72 studies that examine different crossover relationships, there is a gap in the literature regarding whether the job characteristic of high job demands can have a crossover effect on the health levels (i.e. physical and mental health) of the spouse. The first primary goal of this dissertation was to explore the 
impact that one's job experience has on the partner's outcomes. More specifically, the aim was to show how the characteristics of an individual's job (i.e. high demands) impact his or her spouse (i.e. physical and mental health); a direct crossover argument. While research has established a connection between job demands and health of the individual, there is a lack of research examining how job demands cross over to the individual employee's spouse. Further, the common stressors mechanism of crossover will also be addressed by examining the impact that income adequacy levels have on each of the members of the dyad.

Systems Theory stresses the importance of considering the interconnectedness of family members. A systems approach considers the interrelated components of a system. This approach to studying the work-family interface is a holistic view that considers all parts of the system relevant. Hanson (1995) suggests that a system consists of any two or more parts that are interrelated, in that a change in any part of a system results in a change in the other parts. Bronfenbrenner (1977) used Systems theory to make the point that components within a system tend to interrelate and affect each other. Systems theory suggests that processes operating in different settings are not independent of each other. Bronfenbrenner (1977) asserted that systematic work-family research failed to capture the dynamic, interpersonal, social system perspectives. Most work-family research focuses on individual level outcomes. To better understand the complex effects of multiple roles in different domains on family well-being, it is important to examine outcomes at the dyadic level.

Consider the example of a husband spending the day at a job that he feels is very psychologically demanding. The characteristics of the husband's workplace affect his 
health. These work characteristics also send a ripple effect throughout his family system. The job strain that the husband feels may affect his physical health in many ways. If he is too tired to make a healthy dinner, he may stop at a fast food restaurant on the way home and cancel his plans to meet a friend at the gym. His ability to get restful sleep may be affected if he is worried about going to work the next day. The pressure the husband feels may also affect his mental health. He may be depressed or anxious if he feels his work situation is unbearable but for many possible reasons (e.g. financial, geographic) he cannot leave the job. Beyond the numerous negative effects the job characteristics may have on the husband, his wife is an interrelated part of the family system. When the husband returns home, he may discuss work topics with his wife or she may interpret his mood or body language to understand the job strain he is feeling. Considering the intimate and interdependent relationship a husband and wife share, the wife may experience many of the same outcomes as her husband. She may feel empathy for her husband, placing herself in his situation and imagining how it would feel. Further, there may be a financial strain situation that both spouses share, leading the wife to experience many of the same physical and mental health outcomes as her husband.

The second primary goal of the present dissertation was to propose a typology of crossover literature. Although there have been 72 research studies addressing crossover since 1977, there is a lack of uniformity in how literature reviews and crossover results are discussed. Current trends regarding how researchers categorize crossover studies include identifying the key outcome variable in the crossover process (e.g. crossover of depression), the directionality of the effect (e.g. unidirectional), as well as the crossover mechanism responsible for the crossover effect (e.g. direct crossover). Although these are 
important characteristics of crossover studies, the present dissertation suggests several other categorizations that should be incorporated into future discussions of crossover. The review of the crossover literature will be based on this new typology. First crossover of resources are discussed, followed by a review of studies examining crossover of demands. Further distinctions are made regarding the role of resources, stressors or strains in the crossover findings. As will be discussed later, the models hypothesized in this study address two of the categories of crossover research described in the typology (stressor $\rightarrow$ strain; strain $\rightarrow$ strain).

There are several secondary goals that are addressed in the present study.

First, one aim of this study was to replicate the finding of crossover of the strain of health between partners. The inclusion of both a physical health and mental health composite score in the analyses helps clarify the potential crossover relationships between different aspects of health. The present study offers the benefit of examining the crossover between partners of a composite score of both mental and physical health. Whether there is a crossover of the strain of mental health and a crossover of the strain of physical health within the same study population was explored. Second, an advanced method of dyadic data analysis was used that does not try to get around violations of the assumption of independence. Instead, the Actor-Partner Interdependence Model (APIM) embraces the interdependence that is often a natural part of the field of psychology (Cook \& Kenny, 2005).

Third, this dissertation contributes to the field by providing data collected from both partners. This type of data enables a more accurate portrayal of the family system in the research. Further, both partners are employed individuals, thus capturing the dynamic 
of dual-earner couples. Not only is the sample dual-earner couples, these employees are also low-wage grocery employees. As of 2010, there are no crossover studies specifically focusing on a low-wage or hourly population of workers.

One fifth of all U.S. jobs do not provide enough annual compensation to keep a family of four above the poverty level (Waldron, Roberts, \& Reamer, 2004). The employees in the present study are representative of this large percentage of low-wage U.S. workers. They have financial stressors, including transportation and childcare responsibilities, which are made more difficult for those that live paycheck to paycheck (Heymann, 2006). Further, the grocery industry provides a very competitive business environment, making it difficult to maintain profitability because of low profit margins (Plunkett Research Ltd., 2009). As will be discussed more in depth in the Income Adequacy chapter, the mean annual salary for many job titles within the grocery industry is below the poverty level for a family of four (Bureau of Labor Statistics, 2008).

Although research on work and family has greatly expanded, more research is needed on how workplace stress dynamics influence spouse and family health. Relatively few studies examine work stress crossover dynamics, which can occur when job stresses of one working partner transfer over to affect the health of the other partner. Given the abundance of literature supporting the critical role that job demands play in addressing health of employees, it is important to consider the role of these stressors on the health of the family. No studies have directly addressed the potential role of job demands, as defined in the Job-Demand-Control Model, in crossover research. The aim of this study was to address these gaps by examining linkages between perceived job demands of one 
working spouse on the health of the other spouse, as well as to confirm the established crossover relationship of perceived health between spouses.

\section{Contributions}

In summary, this study offers two primary contributions to the literature. First, crossover relationships that have yet to be examined in the literature were hypothesized. Although the connection between job demands and health outcomes has been wellestablished within-person, prior crossover researchers have not examined the potential crossover role these stressors could have on the health of the employee's spouse. Second, a new typology of crossover research is proposed to address the lack of uniformity in how literature reviews and crossover results are reported. The aim of the proposed typology is to add structure to future research and discussions in the crossover literature. Several secondary contributions to the literature are also offered. First, crossover of health between partners was examined, providing the benefit of examining the crossover between partners of a composite score of both mental and physical health. Second, an advanced method of dyadic data analysis was used that does not try to get around violations of the assumption of independence. APIMs embrace the interdependence that is an unavoidable part of studying the impact of job characteristics on the family system. Third, the nature of the sample adds to the literature by examining the understudied population of low-wage dual-earner couples. 


\section{Chapter II}

\section{Crossover Effects}

There are several frameworks that address how stress can be carried over between roles or individuals. According to Bolger et al. (1989), stress contagion exists in two forms: spillover and crossover. First, spillover occurs when stress experienced in one life domain results in stress in another life domain within the same individual, thus an intraindividual transmission of stress. Second, Bolger et al. (1989) discussed crossover as a dyadic, inter-individual transmission of stress or strain, occurring when stress experienced in the workplace by the individual leads to stress experienced at home by the individual's spouse. Crossover involves transmission across individuals, whereby demands and their consequent strain cross over between closely related people in the same social environment (Westman, 2001). Several other terms have been used for crossover, including transmission (Jones \& Fletcher, 1993a; Rook, Dooley, \& Catalano, 1991) and emotional contagion (Hatfield, Cacioppo, \& Rapson, 1994).

This chapter will first discuss the evolving definition of crossover, including gender differences, conditions under which crossover is more likely to occur, and the crossover mechanisms proposed by Westman and Vinokur (1998). Following will be a review and critique of how the crossover literature is currently organized. A proposition will be made for a new typology of crossover research. Finally, a review of all 72 crossover studies to date will be organized via the proposed typology.

\section{Crossover Definition}

Westman (2001) broadened the definition of crossover in several ways. First, instead of crossover exclusively referring to transference between one individual in the 
work domain to the other individual in the home domain, the definition was expanded to include stress and strain experienced at home by the individual leading to stress and strain experienced in the workplace by the spouse. Second, Westman (2001) suggested broadening the concept of crossover to include contagion of positive events in addition to crossover of negative events. According to Westman (2001), similar to the negative impact stressful job demands can have on the partner, positive feelings resulting from positive events may demonstrate a positive crossover effect on the partner's well-being. Third, Westman (2001) stated that crossover at the basic level is a dyadic process that transpires between two individuals. It was suggested that in addition to this principle applying to the dyad, it can be applied to interactions between individuals in the organization.

Gender Differences. Early crossover research, conducted in the 1980s, found that crossover occurs predominantly in the direction from male to female partners (e.g. Bolger DeLongis, Kessler, \& Wethington, 1989; Burke, Weir, \& Duwors, 1980; Fletcher, 1983; Jackson \& Maslach, 1982; Jackson, Zebeck, \& Summers, 1985; Long \& Voges, 1987; Mitchell, Cronkite, \& Moos, 1983; Riley \& Eckenrode, 1986). It has been suggested that women are more likely to take on the burdens of other family members than are men (Howe, Levy, \& Caplan, 2004). It has also been found that women are more likely to be sensitive to the distress of men than the reverse (Cross \& Madson, 1997). Results by Howe et al. (2004) demonstrated that when a man loses his job, the stresses of unemployment are taken on as common burdens by both partners, whereas when a woman loses a job she may face those burdens alone. Kessler and McLeod (1984) showed that events happening to spouses are more distressing for women than for men, 
suggesting the reason for this difference is the greater involvement of women in family affairs.

One limitation of these early studies that demonstrated unidirectional effects from males to females is that participants tended to come from homogeneous and maledominated professions (Crossfield, Kinman, \& Jones, 2005). The occupations studied in the early crossover research were often specific and atypical (Jones \& Fletcher, 1993a). The atypical samples included prison officers (Long \& Voges, 1987), correctional institution administrators (Burke et al., 1980), plant operators (Jackson, Zedeck, \& Summers, 1985) and police officers (Jackson \& Maslach, 1982; Beehr, Johnson, \& Nieva 1995). A second limitation is that wives were often examined as passive recipients of stress and strain from their husbands. Research often did not assess or control for job and life stress of the female partner, some samples mixing populations of working and nonworking wives. For these reasons, these studies cannot rule out the possibility that what appears as direct crossover of stress from husbands to wives is an outcome of wives' job or life stress or of common family stressors of life events affecting both partners (Westman, 2001).

Conditions under Which Crossover Is Likely to Occur. In addition to defining crossover, it is important to consider the conditions under which it is more likely to occur. Bakker, Westman, and van Emmerik (2009) proposed several variables to consider, including when the partner feels empathy, individual differences in susceptibility to emotional contagion, and frequency of exchanging views. Specific to crossover in the workplace, similarity with the source and work climate may also affect the occurrence of crossover. A recent study by Hartel and Page (2009) argued that the 
intensity with which one responds to emotional stimuli may play a role in determining susceptibility to crossover. Specifically, they proposed that individuals high in affect intensity have greater susceptibility.

\section{Crossover Mechanisms}

According to Westman and Vinokur (1998), there are three main mechanisms that lead to crossover within a dyad. Currently, most crossover researchers agree that these proposed mechanisms (e.g. direct crossover, common stressors and indirect crossover) account for the crossover process. In a study by Westman and her colleagues (Westman, Vinokur, Hamilton, \& Roziner, 2004b) all three mechanisms were tested using a sample of dual-earner couples. As I discuss the differentiations between the three crossover mechanisms, I will further report the results of Westman et al. (2004b) to illustrate the definitions.

Direct crossover. According to Westman and Vinokur (1998), direct crossover occurs when the strain of one partner causes an empathetic reaction, increasing the level of stress in the other partner. This mechanism implies direct transmission of stress and strain from one partner to another via empathetic reactions. It is assumed that the emotions expressed by one partner elicit an empathetic reaction in the other partner. Empathy has been defined as sharing another person's feelings by placing oneself psychologically in the person's circumstances (Lazarus, 1991). Research by Jones and Fletcher (1993a) found that most individuals discuss work with their partners and frequently work problems are the main focus of discussion. It was found that although crossover was not related to the amount couples engaged in work discussion, there was a positive correlation between partners' strain measures where work was discussed 
primarily on a routine basis. In the case of health, direct crossover may include more than just empathetic reactions, as spouses may provide care for one another (Vitaliano, Zhang, \& Scanlan, 2003).

Most researchers examine direct crossover without considering whether empathy is the driver behind the crossover effect (for an exception see Bakker \& Demerouti, 2009). Emotional contagion is an alternate explanatory mechanism that has been put forward (Bakker \& Schaufeli, 2000; Westman, Etzion, \& Danon, 2001). Bakker and Schaufeli (2000) describe emotional contagion as an unconscious process in which one individual's emotional state is 'caught' by another person. The person automatically and unintentionally mimics the posture, facial expressions, movements, or voice intonation of the other individual, which leads to emotional assimilation (Hatfield, Cacioppo, \& Rapson, 1994). Empathetic crossover and emotional contagion both address direct crossover between a dyad. The important difference between the two explanatory mechanisms for direct crossover is the level of consciousness. Empathetic crossover is intentional and conscious while emotional contagion is automatic and unconscious.

In order to address the direct crossover mechanism, Westman et al. (2004b) examined whether marital dissatisfaction was transmitted between spouses, hypothesizing an empathetic reaction as the underlying cause of the transmission. Using structural equation modeling, strong support was found for the path of influence of marital dissatisfaction from husbands to wives, although the reciprocal effect of the wives' marital dissatisfaction on their spouses' reported levels was not supported.

Common stressors. The common stressors mechanism implies that crossover may be an outcome of a common stressor affecting the strain of both partners. Those in 
the same household often experience similar stressors stemming from shared

circumstances. What appears to be a crossover effect is the result of common stressors increasing the strain in both partners that share an environment (Westman \& Vinokur, 1998). This mechanism suggests that the relationship between spouses' strain is spurious. Westman et al. (2004b) hypothesized that financial hardship and negative life events of both spouses will increase their marital dissatisfaction, thus being common stressors in the life of the couple. Common stressors and strains were not found to result in the appearance of crossover by having a parallel effect on the strain of both spouses. Financial hardship and negative life events, the common stressors examined in the study, demonstrated differential impact on the partners' marital dissatisfaction, thus not supporting the hypothesized relationship.

Indirect crossover. The mechanism of indirect crossover of strain is an active process of crossover through the behavioral interaction of the partners. One partner's increased stress or strain triggers a negative interaction sequence with the other partner. This explanation of crossover is exemplified by social undermining. In these situations, the stress or strain of one individual leads to social undermining behavior toward the other (Westman \& Vinokur, 1998). Social undermining consists of behaviors that express negative affect, negative evaluation or criticism or hinder attainment of goals (Vinokur \& van Ryn, 1993). These acts serve as a stressor for the recipient of the undermining behavior, causing an increase in his or her level of strain.

To better explain the indirect crossover process, Bakker and colleagues (2008, 2009) recently introduced the spillover-crossover model. According to this model, demands or resources first spill over from work to home, and then cross over to the 
partner. The job demands lead to work-to-family conflict, which in turn, is proposed to lead to conflict with the partner. The negative interaction sequence affects the well-being of the partner. The model has been tested with negative indicators (Bakker, Demerouti, \& Burke, 2009) but has not yet been tested using positive indicators.

Results from Westman et al. (2004b) found that distress resulted in a significant increase in the level of undermining by the other spouse for both the wives and the husbands. Addressing indirect crossover that originates in strains passing through the social undermining mechanism, only the effect on the husbands was significant. For clarification, the increased undermining from the wives toward the husbands resulted in a significant increase in martial dissatisfaction, although increased undermining from the husbands did not have this effect on the wives' marital dissatisfaction.

Co-occurring mechanisms. Some research supports the possibility that several mechanisms may concurrently explain the crossover process (Vinokur et al., 1996; Westman \& Vinokur, 1998). In her proposed framework to guide crossover research, Westman (2001) makes a proposition that crossover may result from several processes that are not mutually exclusive. Based on this proposition, the present dissertation study hypothesizes that both direct crossover and common stressors mechanisms will exist in this population.

\section{A New Typology of Crossover Research}

Although numerous research studies have examined the concept of crossover, there is a lack of uniformity and structure in how researchers discuss the results of crossover research. As a whole, the crossover literature does not fully distinguish between the various approaches researchers have taken to demonstrate crossover effects. 
It is important for crossover researchers to move forward in a more consistent manner and for future research to address the gaps in the crossover literature. Trends regarding how researchers categorize crossover studies include identifying the key variable in the crossover process (e.g. crossover of burnout), the directionality of the effect (e.g. bidirectional), as well as the crossover mechanism responsible for the crossover effect (e.g. direct crossover).

A majority of researchers categorize crossover studies based on the stress, strain, or resource that is either crossed over between individuals or serves as the predictor or outcome in the crossover relationship. For example, in a recent review of crossover research, Bakker, Westman, and van Emmerik (2009) summarized the crossover literature by stating that most studies have investigated and found the crossover of psychological strains such as anxiety, burnout, distress, depression, adjustment, workfamily conflict, and marital dissatisfaction, while some studies examined crossover of health.

Bakker et al. (2009) additionally mentioned the inclusion of both unidirectional and bidirectional crossover studies in the literature. Although the pioneering crossover studies examined effects from the husband to the wife (e.g. Burke et al., 1980; Jackson \& Maslach, 1982; Jones \& Fletcher, 1993a, 1993b, 1996; Long \& Voges, 1987), most recent studies report results as demonstrating either unidirectional or bidirectional crossover.

Another categorization of research involves the origin and destination of crossover. The original definition of crossover only included studies that examined the crossover effects of stress and strain from an individual in the workplace to the partner at 
home. Westman (2001) expanded this definition to account for crossover that originates in the home with one individual and affects the partner in the workplace. Further, with the addition of crossover studies in the workplace, some research addresses situations in which the workplace is both the origin and destination of crossover.

Additionally, an important advancement in the crossover literature was made by Westman and Vinokur (1998). The authors made strides towards clarifying the crossover literature by differentiating between three crossover mechanisms: direct crossover, common stressors and indirect crossover. The next section will discuss these mechanisms in detail. A review by Westman (2001) of the crossover literature was organized broadly based on these three mechanisms. Despite evidence that the three crossover mechanisms need to be considered (Howe, Levy, \& Caplan, 2004; Westman \& Vinokur, 1998) many research articles published since their introduction in 1998 do not mention the mechanisms (e.g. Gareis, Barnett, \& Brennan, 2003; Gorgievski-Duijvesteijn, Giesen, \& Bakker, 2000; Katz, Monnier, Libet, Shaw, \& Beach, 2000; Matthews, Del Priore, Acitelli, \& Barnes-Farrell, 2006; Takeuchi, Yun, \& Tesluk, 2002). It is important for researchers to consider these mechanisms in order to ensure that crossover studies are approached in a consistent manner throughout the literature.

Crossover of resources versus demands. Along with reiterating the importance of describing study results by identifying the key variable in the crossover process, the origin and destination, the directionality, and the crossover mechanisms used, the present dissertation study suggests several other categorizations that should be incorporated into future discussions of crossover. First, it is proposed that researchers should distinguish between crossover of resources and crossover of demands. Regarding crossover of 
demands, Westman (2001) recognized several of the different angles researchers have used to investigate the crossover process. She stated that some researchers had focused on crossover of job stress between spouses, some had examined the crossover of job stress on strain, and others had concentrated on crossover of strain. The present study expands on this idea by proposing a typology that encompasses crossover of resources, as well as crossover of demands.

Westman (2001) suggested broadening the concept of crossover to include contagion of positive events in addition to crossover of negative events. The reasoning behind this expansion of the crossover definition is that positive experiences are qualitatively different experiences than negative experiences (Fredrickson, 2001). Numerous studies have since addressed the crossover of positive experiences between individuals (e.g. Bakker, 2005; Bakker, Demerouti, \& Shaufeli, 2005; Demerouti, Bakker, \& Shaufeli, 2005). In the proposed typology, studies that address a crossover of resources are categorized as a crossover of resources between partners (resource $\rightarrow$ resource) (e.g. engagement predicting satisfaction), resources of one individual affecting the partner's stressors (resources $\rightarrow$ stressor) (e.g. family satisfaction predicting workfamily conflict), or resources of one partner affecting the other's strain (resources $\rightarrow$ strain) (e.g. positive spillover predicting depression). To date, most crossover research has addressed the crossover of negative indicators, such as stressors and strains (Bakker et al., 2009). In the proposed typology, studies that address crossover of demands are categorized as crossover of stressors between partners (stressor $\rightarrow$ stressor) (e.g. work load predicting home load), stressors of one individual affecting the partner's strain (stressor $\rightarrow$ strain) (e.g. work-family conflict predicting physical health), one partner's 
strain affecting the other's stressors (strain $\rightarrow$ stressor) (e.g. depression predicting financial stress) or crossover of strain between partners (strain $\rightarrow$ strain) (e.g. depression predicting physical health). The hypothesized models in the present study address two of the categories described in this typology (stressor $\rightarrow$ strain and strain $\rightarrow$ strain).

\section{Crossover Studies}

There have been 72 research studies that have examined crossover from 1977 to 2009 (see Appendix A). A review of the literature will be structured based on the proposed typology of crossover studies. First, the distinction will be made between crossover of resources and crossover of demands. Research will be discussed in every relevant categorization of crossover studies. If multiple crossover effects are discovered in one study, it is possible that the findings will be discussed under several categories of crossover research. First, all studies addressing crossover of resources will be reviewed. There have been 16 studies in total that have examined crossover of positive indicators at home or in the workplace. This review will be structured based on the organization of crossover literature previously proposed, distinguishing between the roles of resources, stressors and strain in the crossover process. Resources have been defined by Greenhaus and Powell (2006) as assets that may be drawn on when needed to solve a problem or cope with a challenging situation. Researchers have mostly defined job stressors in terms of negative characteristics of the interface between the individual and the organization, measuring stressors like role overload, role conflict, and role ambiguity (Westman, 2001). Jex and Beehr (1991) defined job-related strains as behavioral, psychological or physical outcomes resulting from the stress experience. 
Crossover of resources between dyads will initially be discussed (resource $\rightarrow$ resource), followed by a review of studies addressing a crossover of one partner's resources on the other's stressors (resource $\rightarrow$ stressor), and crossover of resources for one individual affecting the other partner's strain levels (resource $\rightarrow$ strain). Second, all studies addressing crossover of demands will be reviewed. Eight studies have examined the crossover of stressors between partners (stressors $\rightarrow$ stressors). The crossover of one individual's stress on the partner's strain (stressors $\rightarrow$ strain) has been examined in 31 research studies, while only 2 studies have examined the crossover of strain on a stressor (strain $\rightarrow$ stressor). Finally, 22 studies addressing the crossover of strain levels between partners (strain $\rightarrow$ strain) will be reviewed.

Resource to resource. There have been 11 research studies that have discovered crossover effects of resources between partners at home or in the workplace. The studies in this category addressed the concept of engagement or related concepts, as well as participants' satisfaction with their relationship, satisfaction with overall life or job satisfaction. Results supported crossover of resources at home and in the workplace, as well as unidirectional and bidirectional crossover in a variety of samples.

Engagement. Work engagement is a positive, fulfilling, work-related state of mind characterized by vigor, dedication, and absorption (Schaufeli, Salanova, GonzalezRoma, \& Bakker, 2002). Supporting bidirectional direct crossover of engagement, Bakker et al. (2005) discovered a crossover effect for the vigor and dedication components of engagement among partners. Vigor refers to high levels of energy and mental resilience while working, while dedication is characterized by being strongly involved in one's work and experiencing a sense of significance, enthusiasm and 
challenge (Schaufeli et al., 2002). Further, the authors addressed the common stressors mechanism by controlling for several demands and resources in the home domain (Bakker et al., 2005).

In a population of Dutch dual-earner couples, Bakker and Demerouti (2009) recently found a direct crossover effect of women's engagement on their partners. Westman, Etzion, and Chen (2009) also found unidirectional crossover of engagement. Studying a population of business travelers and their spouses, it was found that travelers' vigor, a component of engagement, crossed over to the spouse's vigor. Several studies addressed crossover of engagement in the work environment. Studying a sample of employees and the colleagues they work with most closely, Bakker and Xanthopoulou (2009) found that the crossover of work engagement, and particularly vigor, takes place only on days when colleagues interact more frequently than usual. Bakker, van Emmerik, and Euwema (2006) discovered a unidirectional crossover of engagement in a population of Dutch constabulary officers working in teams. Team level work engagement crossed over to affect the individuals' engagement levels. Bakker (2005) found a unidirectional direct crossover effect in a population of music teachers and students. It was found that the more flow experiences (similar concept to engagement) that teachers reported, the higher the frequency of comparable experiences in their students.

Satisfaction. Multiple studies have measured resources such as satisfaction with a component of life. In a recent study, Bakker et al. (2009) found bidirectional direct crossover of relationship satisfaction in a population of Dutch dual-earner couples. Also finding bidirectional direct crossover of a resource, results from Prince, Manolis, and Minetor (2007) demonstrated a crossover of life satisfaction between married couples. A 
separate study additionally found crossover of life satisfaction, although only from the male to the female partner (Demerouti, Bakker, \& Schaufeli, 2009). Addressing crossover from the couple to an individual, Hammer et al. (2005b) demonstrated a significant positive relationship between use of alternative work arrangements by the couple and the wives' job satisfaction. Similarly, in the earliest study to address crossover of resources, Greenhaus and Parasuraman (1987) found that wife's employment was positively related to husband's job satisfaction, as well as quality of life.

Resource to stress. Only two research studies have examined the crossover of resources for one individual on stressors of the partner. Both of these crossover findings relate to how work-family issues and support for childcare affect female partners. In a study of dual-earner couples, Hammer et al. (2005b) found that the use of dependent care supports by the couple was associated with high levels of work-to-family and family-towork conflict of the wife. Results by Kossek, Pichler, Meese, and Barratt (2008) demonstrated a unidirectional direct crossover effect from child-care provider to mother. Mothers using care from providers who reported higher quality parent-caregiver social relationships reported lower work-family conflict.

Resource to strain. Four studies have supported a crossover effect of one individual's resources on the partner's strain. All the studies that fall into this category demonstrated a unidirectional direct crossover effect of resources on the well-being of one individual in the dyad. Hammer et al. (2005a) found two separate unidirectional crossover effects in a sample of dual-earner couples. Results demonstrated that husbands' work-to-family positive spillover was related to reduced depression levels for wives over time. Similarly, it was shown that wives' family-to-work positive spillover was related to 
reduced depression in husbands over time. Several unique samples also fall into this category. In a sample of 82 naval couples, Morrison and Clements (1997) found that the return of the male naval officer from deployment was associated with elevated mental health of the female partner. Similarly addressing mental health, Kossek et al. (2008) demonstrated that a higher quality relationship between the child-care giver and the parent was associated with lower depressive symptoms for the mother. Finally, Beehr, Johnson, and Nieva (1995) found a crossover effect for police officer's coping strategies on the elevated well-being of the partner.

Summary of studies examining crossover of resources. Overall, a majority of the studies addressing the role of resources in the crossover process have been recently conducted (e.g. Bakker \& Demerouti, 2009; Bakker et al., 2009; Kossek et al., 2008; Westman et al., 2009). It appears that researchers took heed of the suggestion made by Westman (2001) to consider the role of positive indicators in the crossover process. Although the present dissertation study does not address the crossover of resources, there are some findings that are helpful in lending support for the use of measures of wellbeing. Several research studies found significant crossover of resources affecting mental health and well-being (Beehr et al., 1995; Hammer et al., 2005a; Kossek et al., 2008; Morrison \& Clements, 1997), which is an outcome of interest in the present study. Further, multiple studies examining the crossover of resources demonstrated a bidirectional direct crossover effect between a male and female partner (Bakker et al., 2009; Bakker et al., 2005; Prince et al., 2007), lending support to the argument that it is important to consider both partners in the crossover process. The present study hypothesizes bidirectional crossover between male and female spouses. 
Stressor to stressor. There have been eight studies that have addressed the crossover of stressors between partners. Four of these studies examine how work-family conflict affects the dyad, all finding support for bidirectional direct crossover. Other studies that addressed the crossover of stressors generally examined how general stress at work affected the partner's stress level at home. Westman and Etzion (1995) found bidirectional crossover of sense of control in a sample of male Israeli military officers and their working wives. Bolger et al. (1989) discovered a bidirectional crossover effect between one individual's stress at work and the other partner's stress at home. Similarly, results from Dikkers et al. (2007) demonstrated that when husbands' reported a higher work load, their wives experienced a higher home load. Also demonstrating unidirectional crossover, Barnett, Gareis, and Brennan (2008) showed that men whose wives worked evenings (compared to day shift), reported more work-family conflict, although only when the wives also worked long hours.

Work-family conflict. Hammer, Allen, and Grigsby (1997) found that an individual's level of work-family conflict was a significant predictor of his or her partner's level of work-family conflict. This finding was later supported by several other research studies. Westman and Etzion (2005), studying women in the Air Force and their working spouses, discovered a significant crossover effect for both work-to-family conflict, as well as family-to-work conflict. In a sample of 60 Israeli married couples, Cinamon, Weisel, and Tzuk (2007) found that work-to-family conflict of one spouse was positively correlated with family-to-work conflict for the other spouse. In a recent study, van Emmerik and Peeters (2009) replicated this crossover effect in the work environment. Results demonstrated that team-level work-to-family conflict had a 
crossover effect on individual-level work-to-family conflict. Further, team-level familyto-work conflict was shown to significantly cross over to the individual's level of familyto-work conflict.

Stress to Strain. The most studied relationship between stressors or strains in the crossover literature is the examination of how stressors affecting one individual cross over to affect the strain levels of his or her partner (e.g. negative health outcomes, vitality, social functioning). Thirty-four research studies have examined the crossover effects of stressors on strain. Results demonstrate both unidirectional and bidirectional crossover relationships with studies showing support for the direct, indirect and common stressors crossover mechanisms. Various work or life stressors of one individual were shown to have a crossover effect on strains including well-being (mental and physical health), satisfaction (with job, life, family, or marriage), burnout, and other variables such as mood, withdrawal, emotional adjustment, and relationship tension.

Mental health. Several researchers examined the effect that women's employment has on their spouses' mental health. Rosenfeld (1980) found that wives' employment was positively related to husbands' distress. Results from Rosenfeld (1992) demonstrated a similar crossover effect, showing that wives' employment was negatively related to husbands' mental health, although only when the employment decreased husbands' relative income and increased his share of domestic labor. Conversely, Roberts and O'Keefe (1981) found that the employment status of the wife had no significant effect on the husband's depression. Several additional studies demonstrated a unidirectional direct crossover from the female partner's stress to the male partner's mental health. In a sample of female reduced-hours physicians and their full-time 
employed husbands, Gareis, Barnett, and Brennan (2003) found that wives' ratings of their own work schedule fit predicted husbands' psychological distress. Similarly, Crossfield, Kinman and Jones (2005) demonstrated positive relationships between women's work stressors and the anxiety and depression reported by their male partners in dual-earner couples.

A number of researchers have found unidirectional direct crossover effects for the male partner's stressors on the female's mental health levels. Hammer et al. (2005a), in a longitudinal study of dual-earner couples, found that husbands' work-family conflict was significantly related to wives' depression over time. Results from Mitchell et al. (1983) demonstrated that husbands' negative events were related to wives' depression. Similarly, Riley and Eckenrode (1986) found that women with lower levels of personal resources were distressed by the negative life events of their significant others. In a sample of naval couples, Morrison and Clements (1997) found that a high level of male's role ambiguity was associated with the decreased well-being of females. In a sample of working couples, Jones and Fletcher (1993a) demonstrated a crossover of male's work demands on female's psychological health. Results showed that husbands' interpersonal work situation was correlated with wives' anxiety and depression, while husbands' stressors related to supports at work was correlated to wives' depression. Rook, Dooley, and Catalano (1991) found crossover of husbands' work stress, finding stressors were associated with significantly elevated psychological distress symptoms in wives. Burke et al. (1980) found a similar crossover effect in a population of Senior Administrators of correctional institutes and their spouses. It was found that husbands' job demands were related to increased psychosomatic symptoms and negative feeling states of wives. Long 
and Voges (1987) also found crossover of husbands' job stress on wives' psychological well-being, using a sample of prison officers and their wives

Only a few research studies demonstrated bidirectional crossover of stress on strain between partners. In a daily diary study, Jones and Fletcher (1993b) found that daily fluctuations in work stressors of one individual crossed over to the psychological, as well as physical, health of the spouse. Haines, Marchand, and Harvey (2006), studying almost 3,000 dual-earner couples, demonstrated that one individual's experience with workplace aggression was related to higher levels of psychological distress in the partner. Haines et al. (2006) additionally accounted for the common stressors mechanism of crossover by controlling for other possible stressors including decision authority on the job, working hours, irregular shifts and marital strain. A study by Westman and Vinokur (1998) supported the common stressors and indirect mechanisms of crossover, demonstrating that life events were a common stressor affecting depression. Social undermining also was found to mediate the process.

A study by Howe, Levy, and Caplan (2004) similarly accounted for multiple crossover mechanisms. In a sample of unemployed individuals and their partners, it was found that secondary stressors after job loss for the job seeker are associated with increases in depressive symptoms for his or her partner. Further, results demonstrated an indirect crossover effect as the secondary stressors were shown to degrade the quality of the couples' relationship, which in turn contributed to increased distress in the job seeker's partner. This relationship can also be considered a crossover of stress on strain. Regarding the common stressor mechanism, Howe et al. (2004) found that emotional reactions in the two members of the couple are not independent and may be mutually 
reinforcing. Results by Vinokur, Price and Caplan (1996) also supported the Common Stressors and Indirect Crossover mechanisms. It was found that partner's depression is affected by financially-related common stressors, as well as the stress of undermining transactions.

Physical health. Only two studies have discovered bidirectional direct crossover of one individual's stressors on the physical health of the partner. As previously mentioned, Jones and Fletcher (1993b) discovered that one spouse's daily fluctuations in work stressors cross over to affect the physical health measures for the other spouse. Research by Westman, Keinan, Roziner and Benyamini (2008) supported the indirect and common stressors mechanisms of crossover with the finding that financial hardship affected perceived health directly, as well as indirectly via undermining. Two studies found a unidirectional direct crossover effect of wives' stressors on husbands' physical health strain levels. Haynes, Eaker, and Feinleib (1983) demonstrated that husbands of women in white-collar jobs were over 3 times more likely to develop Coronary Heart Disease than those married to housewives or blue-collar workers. Addressing another objective health measure, Jones and Fletcher (1996) found that wives' domestic stressors were correlated with husbands' sleep for the subsequent night.

Three crossover studies have discovered a unidirectional direct crossover effect from the male partner's stressors to the female partner. Analyzing over a million cases of death, Fletcher (1983) demonstrated a crossover effect of husbands' job risks on the life expectancy of the wife. In a similar study, Fletcher (1988) showed that occupational mortality risk of husbands affects wives' life expectancy and cause of death. Eckenrode 
and Gore (1981) found that the frequency of significant others' life events affected women's health status and health behaviors.

Satisfaction. Only one study found a bidirectional direct crossover effect of one partner's stressors on the other's satisfaction. Brockwood, Hammer, Neal, and Colton (2001) found that the extent to which one's spouse made accommodations at home had a significant negative impact on one's own level of family satisfaction for both members of dual-earner couples. Several studies have demonstrated a unidirectional crossover effect of female's stressors on male's satisfaction. In another pioneering study examining the effects of female employment, Booth (1977) found that wives' employment had no effect on husbands' marital satisfaction. Conversely, Staines, Pottick, and Fudge (1986) found that husbands of employed wives had lower job and life satisfaction than husbands of housewives. Parasuraman, Greenhaus, and Granrose (1992) did find a significant relationship between female's family role stressors and the male spouse's family satisfaction. In the only bidirectional study originating with the male's stressors, Burke et al. (1980) found that husbands' job demands were related to less marital and life satisfaction in wives.

Several studies by Jackson and colleagues addressed how husbands' job stressors can affect satisfaction with the wives. In a sample of police officers and their spouses, Jackson and Maslach (1982) found crossover of husbands' job stress on the dissatisfaction and distress of the wife. Jackson et al. (1985) examined plant operators and their spouses, finding the emotional interference caused by the husbands' job experiences was related to wives' dissatisfaction with the husbands' job, as well as their quality of life. 
Other strains. A variety of other operationalizations of strain have been used when addressing crossover effects in research. Several researchers have examined the crossover effects of stress on burnout. In a sample of Certified Public Accountants and their spouses, Pavett (1986) found that CPA's stress affected spouses' physical and psychological symptoms of burnout. Westman and Etzion (1995) found a bidirectional direct crossover effect of the male partner's sense of control on the female partner's burnout levels. A few studies have addressed the crossover effects of stress on the partner's assessment of the relationship. In a sample of dual-earner couples, Gareis et al. (2003) found that husbands' ratings of their own work-schedule fit were predictive of their wives' rating of marital role quality. Matthews, Del Priore, Acitelli, and BarnesFarrell (2006) used the Actor-Partner Interdependence Model (APIM), finding a direct crossover effect for women's work-to-relationship conflict on men's reports of relationship tension.

Demonstrating several unidirectional crossover relationships, Hammer, Bauer, and Grandey (2003) found that work-family conflict had a crossover effect on withdrawal behaviors. Results demonstrated that husbands' interruptions at work were predicted by wives' family-to-work conflict. Further, husbands' family-to-work conflict was predictive of wives' lateness at work. In another unidirectional crossover finding, Katz et al. (2000) studied a population of medical student marriages, discovering that medical students' perceived stress was significantly associated with their spouses' emotional adjustment. Finally, Lavee and Ben-Ari (2007), in a sample of Israeli dual-earner couples, found a direct crossover effect from one's work stress to partners' mood. 
Strain to stressor. Only two studies have examined crossover effects of one partner's strain on the other's stressor levels. Both research studies in this category found direct crossover effects from the husband to the wife. Results from Dikkers et al. (2007) demonstrated that when husbands reported more psychological health complaints, their wives experienced higher home load. Studying a population of Dutch dairy farm couples, Gorgievski-Duijvesteijn et al. (2000) found that husbands' health complaints were predictive of the couples' financial problems 10 years later.

Strain to Strain. There have been 22 research studies that have examined crossover of strain between dyads in both the work environment as well the home environment. A majority of the studies in this category address crossover of the same strain in both individuals. For example, there have been eight studies examining crossover of burnout, most of these findings support bidirectional crossover of burnout between partners or coworkers. Another common strain that has been examined in this category of crossover research is mental health or depression. Other strain variables that have been examined in the crossover context include job-induced tension, marital satisfaction, general adjustment, physical health and mood.

Burnout. Six research studies have found evidence for a bidirectional direct crossover relationship of burnout, four of these using employee populations. Bakker, LeBlanc, and Shaufeli (2005) examined a population of intensive care nurses from 12 European countries. It was found that nurses who reported the highest prevalence of burnout among their colleagues were most likely to experience high levels of burnout themselves. Perceived burnout complaints had a positive independent impact on each of the three burnout dimensions. In another occupational setting, Bakker and Schaufeli 
(2000) demonstrated bidirectional crossover of burnout in a population of Dutch high school teachers. It was additionally found that the prevalence of burnout among colleagues had the strongest effect on individual teachers' burnout when the teachers were highly susceptible to others' emotions and when they frequently communicated work-related problems with each other. Bakker, Schaufeli, Sixma and Bosveld (2001) found similar results in a population of general practitioners, susceptibility to emotions expressed by others also had a moderating effect on the relationship between burnout among colleagues and individual's emotional exhaustion component of burnout. With samples of both soldiers and teachers, Bakker, Westman, and Schaufeli (2007) supported direct bidirectional crossover of burnout via a process of empathy between an individual and his or her colleague. Results also demonstrated a moderating effect for similarity with the stimulus person. One workplace crossover study of burnout that found a unidirectional effect was conducted by Bakker et al. (2006). In a sample of Dutch constabulary officers and their wives, it was discovered that team level burnout was related to individual team members' burnout.

Two studies have found bidirectional crossover of burnout between partners. Bakker et al. (2005) found a crossover of the emotional exhaustion and cynicism components of burnout among partners working in a variety of occupations. In an attempt to rule out common stressors, they additionally controlled for several demands at home. Westman and Etzion (1995) also found bidirectional crossover of burnout in a sample of male military officers in Israel and their working wives. Another Israeli study only supported crossover of burnout from husbands to wives (Westman, Etzion, \& Danon, 2001). 
Mental health. There have been five research studies that have addressed the bidirectional crossover of depressive symptoms, all of which have demonstrated direct crossover between partners. Katz, Beach, and Joiner (1999), using a sample of undergraduate dating couples, found that partners' levels of depressive symptoms were related even after controlling for relationship satisfaction. Several studies found a bidirectional crossover of psychological health between married couples (Cronkite \& Moos, 1984; Dikkers et al., 2007). Westman and Vinokur (1998) examined a population of male veterans and their partners, finding evidence for all three crossover mechanisms. A bidirectional direct crossover of depression between partners was demonstrated. Further, life events were shown to serve as a common stressor in the crossover process, while social undermining played a mediating role. The former two crossover findings can be qualified as stressors affecting strain. Finally, Mitchell et al. (1983) found a unidirectional direct crossover effect of husbands' strain levels on wives' depression.

Other strain. Several studies examined crossover of health between partners. Westman et al. (2008) found bidirectional crossover of perceived health in a sample of Russian couples, as well as supporting the indirect and common stressors mechanisms of crossover. In a population of Dutch dairy farm couples, Gorgievski-Duijvesteijn et al. (2000) found that husbands' health complaints were predictive of the wives' health complaints 10 years later. In another unidirectional finding originating with the male partner, Westman, Vinokur, Hamilton, and Roziner (2004) found direct crossover of marital dissatisfaction from Russian army officers to their wives. Takeuchi, Yun, and Tesluk (2002) also demonstrated unidirectional direct crossover of strain in their sample 
of Japanese expatriates and their spouses. Reciprocal crossover effects between spouse and expatriate adjustment were found.

Several unidirectional direct crossover relationships of strain have been demonstrated from the female partner to the male partner in populations of dual-earner couples. Demerouti, Bakker, and Schaufeli (2005) found a crossover path from females' exhaustion to males' exhaustion on the job. In one study that did not examine crossover of the same strain between partners, Chan and Margolin (1994) demonstrated a crossover effect of wives' negative mood and work fatigue on the husbands' reactions at home. Further, it was shown that wives' home affect had a significant relationship with husbands' work mood. Finally, in a unique sample of Israeli school principals and teachers, Westman and Etzion (1999) found significant crossover of job-induced tension between principles and the teachers, and vice versa. One study addressing indirect crossover found unidirectional crossover from partners to a sample of faculty members at a university (Greene, Bull Shaefer, MacDermid, \& Weiss, 2010). It was found that negative emotional displays of the partner had a positive significant effect on faculty members' turnover exploration activities as well as a negative effect on their career resilience.

Summary of studies examining crossover of demands. One of the main goals of the present dissertation is to examine the crossover of demands between partners. This review discussed several methods to address the crossover of demands, including crossover of stressors, crossover of stressors on strain, crossover of strain on stressors, and finally, the crossover of strain. Although a majority of the reviewed studies focused on the traditional male and female partnership, there is a growing body of literature that 
examines crossover in the workplace (see Bakker et al., 2005; Bakker \& Schaufeli, 2000; van Emmerik \& Peeters, 2009; and Westman \& Etzion, 1999 for examples). The relationship that most researchers were interested in and found support for was the crossover of stressors on strain in couples (e.g. partners or spouses) or between coworkers; with almost half of the crossover studies in the literature examining this relationship. Extensive support has similarly been found for the crossover of strain between partners, with almost a third of the studies examining this crossover effect. The

present study also addresses the crossover of demands (i.e. stressor $\rightarrow$ strain and strain $\rightarrow$ strain).

\section{Overview of Present Crossover Study}

The relationships of interest in the present study are in line with the majority of crossover research examining either crossover of stressor on strain or crossover of strain between partners. Several crossover relationships were hypothesized that examine the effect of one partner's stressors on the other's strain, as well as crossover of strain between partners. Specifically, it was hypothesized that one individual's level of job demands would cross over to affect the spouse's health (i.e. physical and mental health) (stressor $\rightarrow$ strain). The present dissertation study was also interested in the crossover of strain between partners. It was hypothesized that levels of physical health, as well as levels of mental health, would be correlated between spouses (strain $\rightarrow$ strain). Addressing the common stressors mechanism of crossover, it was hypothesized that ratings of income adequacy would serve as common stressors affecting well-being (i.e. physical and mental health). This hypothesis could also be considered an examination of the crossover of stressors on strain. 
Many of the reviewed studies provide support for the present study's hypotheses. Along with the strong support demonstrated for bidirectional direct crossover of one partner's stressors on the other's strain and bidirectional direct crossover of strain between partners, some of the constructs of interest in the present study have been supported in the literature (e.g. crossover effects on health and well-being). In the current study, a stressor is measured by job demands, one of the variables proposed in Karasek's (1979) Job Demand-Control Model. No studies to date have examined the potential crossover effects that job demands have on physical and mental health measures. A majority of researchers have examined the crossover of work demands by investigating how the many different forms of stress resulting from one individual's job affects the partner (for examples see Bolger et al., 1989; Crossfield et al., 2005; Dikkers et al., 2007; Jackson \& Maslach, 1982; Jones \& Fletcher, 1993a and 1993b; Lavee \& Ben-Ari, 2007; Long \& Voges, 1987; Morrison \& Clements, 1997; Pavett, 1986; Rook et al., 1981; Westman \& Etzion, 1999). The importance of job demands in relation to health will be discussed in the following section.

Although no studies have examined the crossover of job demands, researchers have demonstrated crossover effects of stress on both mental and physical health. Crossover of stress on mental health (Crossfield et al., 2005; Eckenrode \& Gore, 1981; Gareis et al., 2003; Hammer et al., 2005a; Howe et al., 2004; Jones \& Fletcher, 1993a and 1993b; Mitchell et al., 1983; Rook et al., 1991) have been established. Similarly, crossover of stress on physical health have been demonstrated in the crossover literature (Eckenrode \& Gore, 1981; Fletcher, 1988; Gorgievski-Duijvesteijn et al., 2000; Jones \& Fletcher, 1993b). 
Providing support for the hypothesized direct crossover of strain between partners, researchers have demonstrated direct crossover of physical and mental health (Cronkite \& Moos, 1984; Dikkers et al., 2007; Gorgievski-Duijvesteijn et al., 2000; Katz et al., 1999; Vinokur et al., 1996; and Westman \& Vinokur, 1998). The common stressors mechanism of crossover (Westman \& Vinokur, 1998) suggests the crossover effect is in fact the outcome of characteristics of the shared environment, such as common stressors, which increase strain in both partners. The common stressor of income adequacy will be examined based on the findings of Westman et al. (2008) that confirmed economic hardship played a role as a common stressor affecting crossover. 


\section{Chapter III}

\section{Job Demands and Health}

In the early part of the twentieth century, researchers designed jobs using the principles of scientific management (Morgeson \& Campion, 2003). It was thought that the division of labor would increase efficiency and productivity of employees (Taylor, 1911). This approach focused on specialization and simplification of jobs in order to decrease training needs and staffing difficulties. These jobs did not meet human potential or higher order needs, leaving work that was tedious, boring and repetitive. This led organizational researchers to pay attention to the environment in which employees work and how job design affects employee motivation. Researchers began to focus on the job characteristics that could enhance the job satisfaction and intrinsic motivation of employees (Morgeson \& Campion, 2003). One of the primary theoretical models that had addressed this idea of job enrichment is the Job Characteristics Theory (Hackman \& Oldham, 1976).

\section{Job Characteristics Theory}

The Job Characteristics approach to job design suggests that five job characteristics produce critical psychological states in the employee, eventually leading to positive work outcomes (Hackman \& Oldham, 1976). The five characteristics are skill variety, task identity, task significance, autonomy, and feedback. The first three job characteristics are suggested to increase the meaningfulness of work. Skill variety refers to the degree to which the job requires a variety of different activities, providing the employee opportunities to use different skills and talents. Task identity involves the extent to which the worker feels responsible for a meaningful and whole part of the work. 
The final characteristic contributing to meaningfulness of work, task significance, refers to the impact the job has on the lives of others.

The fourth job characteristic, autonomy, refers to the degree to which the job provides freedom and independence in carrying out the work (Hackman \& Oldham, 1976). This characteristic of the job is expected to contribute to the psychological state of increased responsibility for work outcomes. The final characteristic, feedback, refers to the degree to which carrying out the work activities required by the job results in the individual obtaining direct and clear information about the effectiveness of his or her performance (Hackman \& Oldham, 1976). Feedback is expected to provide knowledge of the results, the third critical psychological state. Job dimensions influence personal and work outcomes by operating through the critical psychological states. The model postulated that the outcomes of the job characteristics include high intrinsic motivation, high job performance, high job satisfaction, low absenteeism and turnover (Fried \& Ferris, 1987; Hackman \& Oldham, 1976).

The movement towards a focus on job design paved the way for researchers to examine additional aspects of the work environment that may affect employees. Karasek (1979) proposed a model that suggested that psychological strain resulted from the joint effects of the demands of work situations and the decision-making freedom or discretion the employees facing the demands were given. Since this wave of research began, it has been widely suggested that work design is a major determinant of employee health and effectiveness (Holman \& Wall, 2002). 


\section{Job Demands}

Research has established the connection between the work role and the family role, showing that demands in one domain spillover and affect the other domain (Frone, 2003). It has been further demonstrated that the demands stemming from the work role can have a negative impact on the health of the employee (Bellavia \& Frone, 2005). Fox, Dwyer, and Ganster (1993) defined job demands as psychological stressors, such as working fast and hard, having a lot to do, not having adequate time, and existence of conflicting demands. The authors stressed that these are not physical demands, giving the example that a fast, hectic work pace could lead to anxiety about maintaining that pace and the potential consequences of not completing the work. Research has demonstrated that job demands are strongly linked with the health of employees (Fox, Dwyer \& Ganster, 1993; Parkes, Mendhamm \& Von Rabenau, 1994; Stansfeld, Bosma, Hemingway \& Marmot, 1998; van der Doef \& Maes, 1999). One of the models connecting job demands to health is the Job Demand-Control Model. The model backs up the hypothesis that job demands are one of the important predictors to consider when measuring health outcomes.

Job Demand-Control-Support Model. Karasek's (1979) Job Demand-Control (JDC) Model is a leading stress model in Occupational Health Psychology and one of the most influential models connecting work and health (van der Doef \& Maes, 1999). According to this framework, a combination of job demands and job control determine the psychological work environment. Job demands refer to work stressors or the workload and are often operationalized in terms of time pressure, role overload or other role conflicts (van der Doef \& Maes, 1999). As mentioned previously, job demands can 
be defined as psychological stressors, such as working fast and hard, having a lot to do, not having adequate time, and existence of conflicting demands (Fox et al., 1993). Job control refers to the ability to control work activities, and as a concept, is primarily measured by assessing decision latitude (Karasek, 1979). Decision latitude consists of two components: decision authority and skill discretion (van der Doef \& Maes, 1999). Some researchers have redefined the model with the addition of work-related social support. The Job Demand-Control-Support (JDCS) Model has basis in the finding that social support may modify the impact of demands on and off the job (Johnson \& Hall, 1988).

According to the JDCS Model, there are four basic situations that can occur. These situations are categorized as relaxed, passive, active, and strain, and each of these situations is a combination of job demand and job control levels (Karasek, 1979). Relaxed situations, considered ideal, are characterized by low demands and high control. Low demands matched with low levels of control constitute passive situations, while active situations consist of high demands and high control. One of the two major assertions of the model involves the final category, high strain situations. Work situations in which demands are high and control is low result in psychological stress reactions such as job strain and negative health effects. The strain hypothesis states that individuals working in high strain jobs (high demands and low control) experience the lowest wellbeing. In the iso-strain hypothesis of the JDCS model, jobs characterized by high demands, low control, and low support (iso-strain) are considered to be the work situations most detrimental to employee health. 
Strain Hypothesis. The focus of this hypothesis is whether the most negative outcomes are found in employees in the high-strain situation (van der Doef \& Maes, 1999). The strain hypothesis has been operationalized as both additive and interactive effects of demands and control, demonstrating a lack of consistency in the literature (de Lange et al., 2003). As van der Doef and Maes (1999) stated, "close inspection shows that the empirical tests of the JDC(S) model do not all examine the same hypothesis (pp. 87)." Researchers often do not indicate whether the negative outcomes are the result of additive or interactive effects, leading to uncertainty about whether the negative effects could be solely attributable to either demand or control levels. A review by de Lange et al. (2003) addressed this issue by proposing that both additive and multiplicative interaction effects be considered as support for the strain hypothesis, as long as the workers in the high demands/low control condition experience the highest levels of strain. Karasek (1989) claimed that the existence of a multiplicative interaction term is not the primary issue in the JDC model. Other researchers (e.g. Ganster, 1989) have maintained the view that the moderating effect of control is the main thrust of the model.

Buffer Hypothesis. Aside from the strain hypothesis, the second dominant research idea regarding the JDC Model is the buffer hypothesis (van der Doef \& Maes, 1999). This hypothesis states that control can moderate the negative effects of job demands on well-being. Unlike the strain hypothesis, the buffer hypothesis explicitly predicts an interactive effect of demands and control, in which control moderates the effects of demands on the outcomes (van der Doef \& Maes, 1999). When considering the JDCS Model, an interaction is predicted between demands, control and support, 
indicating a buffering effect of support on the negative impact of high strain on psychological well-being (van der Doef \& Maes, 1999).

The two ideas are not mutually exclusive: the buffer hypothesis can be interpreted as a specification of the strain hypothesis (van der Doef \& Maes, 1999). The practical implications of these two hypotheses differ, however. Evidence for moderating effects of control may lead to recommendations to increase job control, without consideration for the level of demands. If additive effects of demands and control are valid, this strategy would not be effective since high demands would maintain their detrimental effect on the employees. Research evidence for the buffer hypothesis has been inconsistent (van der Doef \& Maes, 1999).

Research has largely supported the prediction that strain occurs when demands are high and control is low, suggesting that the JDC model correctly identifies the central aspects of the psychological work environment (van der Doef \& Maes, 1999; Taris, 2006). A review of high-quality studies found that $63 \%$ reported significant main effects of job demands in predicting physical and psychological indicators of strain (usually in conjunction with main effects of control) (de Jonge et al., 2000). Conversely, even highquality studies only provide modest support for the interactional effect of demands and control (de Lange et al., 2003).

As Taris (2006, pp. 100) asserted, “...there is no question that the demand-control model is correct in identifying demands and control as major predictors of work stress and ill-health, this does not seem to apply to the idea that demands and control interact in affecting these outcomes." The proposed study is interested in the main effects of job demands, but does not address potential interactions proposed in the JDCS Model based 
on the lack of support for the buffer hypothesis. It has been well-established that job demands have an effect on the health and well-being of the individual. Research has also demonstrated the crossover effects that work stressors can have on the individual's spouse. Few research studies have examined the impact of job demands as specific work stressors on the health of the individual's spouse. The proposed dissertation study will address this gap in the literature. The following section will discuss in more detail how the outcomes of health will be addressed and summarize research connecting job demands and health within person. 


\section{Chapter IV}

\section{Health}

Based on research that suggests physiological and psychological health are important outcomes to consider in relation to psychological stressors, the present study examines the physical and mental components of overall health (Jex \& Crossley, 2005). Many researchers have examined health as an outcome of stress at work, operationalizing the term in numerous ways. To better understand the range of health outcomes examined in organizational research, I will first briefly address some of the common operationalizations of physical health, followed by a review of mental health outcomes commonly examined.

\section{Self-Report versus Objective Measures}

Physical health can be addressed using self-report measures or objective indicators. Objective measures of physical health include diagnosed health problems, health care utilization, sick days, worker's compensation claims, sleep patterns, occurrence of autoimmune disease, as well as physiological indicators like catecholamines (measured via venous plasma adrenaline and urinary excretions), heart rate and blood pressure (when asleep and when active), and coagulation factors (Belkic, Landsbergis, Schnall, \& Baker, 2004; Jex \& Crossley, 2005; Schnall, Landsbergis \& Baker, 1994; Segerstrom \& Miller, 2004; Theorell \& Karasek, 1996; Totterdell, 2005). Examples of self-report measures of physical health include report of psychosomatic symptoms, measures of cardiovascular disease risk (e.g. report of age or weight) (Belkic et al., 2004). Many researchers use single-item self ratings of health to address well-being (Bowling, 2005). Most measures of mental health involve self-report data; to utilize 
objective data researchers may use clinical diagnoses of anxiety or depression-related disorders. To gather self-report mental health data, scales addressing anxiety, depression, distress or general well-being are often used (Crossfield et al., 2005; Griffin et al., 2007; Jex \& Crossley, 2005). The present dissertation study uses self-reports of physical health and mental health among employees and their spouses.

\section{Health as Measured in the Present Study}

The present study addresses health functioning as an outcome, measuring two components: a physical health summary and a mental health summary (Ware, Kosinski, $\&$ Keller, 1996). General health is measured via computation of composite scores for physical and mental health. Higher scores indicate better functioning either physically or mentally (Ware, Kosinski, \& Keller, 1996). Although numerous antecedents of health in the workplace have been examined, the present study is interested in the role of job demands in predicting these employee health scores. Specifically of interest are the crossover effects of the job demands experienced by one spouse on the health component scores of the other spouse, as well as the crossover effects of health between spouses. Next, I will review research that has examined various predictors of overall health or operationalizations of the health components, followed by a more detailed discussion of the connection between the predictors of interest and overall health. Crossover studies involving health outcomes were discussed in the Crossover chapter of this dissertation proposal.

\section{Predictors of Health in the Workplace}

Researchers have demonstrated that many organizational factors have an impact on employee health rates. Many of the predictor variables discussed involve fairness of 
treatment or role stressors that lead to increased strain for employees, in turn affecting their health. Studies have shown that organizational justice has an impact on health of employees (Elovainio, Kivimaki, \& Vahtera, 2002; Kivimaki, Elovainio, Vahtera, \& Ferrie, 2003; Taris, Kalimo, \& Shaufeli, 2002; Tepper, 2001). Cropanzano (2005) discussed the relationship between justice and stress, suggesting that a way for executives to address employee health concerns is to mitigate stressful situations rooting from injustice. This idea is exemplified in research by Elovainio et al. (2002). When examining procedural and relational justice, it was found that the rates of absence due to sickness were significantly higher for those reporting low perceived justice than those that perceived high justice at work (Elovainio et al., 2002).

Work variables that involve the family dimension have similarly been shown to have an effect on health of employees. Researchers have demonstrated a connection between the role stressor of work-family conflict and health. Bellavia and Frone (2005) discussed the outcomes of work-family conflict on the individual as including mental health, well-being, physical health, satisfaction with life, stress, substance use, emotional exhaustion, obesity and mood disorders. Allen and Armstrong (2006) established a connection between work-family conflict and health behaviors. It was found that familyto-work conflict was associated with less physical activity and eating more high fat foods, while work-to-family conflict was related to eating fewer healthy foods. In a longitundinal study, Frone, Russell and Cooper (1997) connected family-to-work conflict with higher levels of depression, poor physical health and incidence of hypertension. Addressing several work-family variables, research by Hammer et al. (2005a) 
demonstrated that work-family positive spillover had a stronger impact on depression than work-family conflict.

Health is often impacted by the way employees are treated by others at work. Thomas and Ganster (1995) found that supportive supervisors, as well as flexible scheduling, had positive effects on perceptions of control, which in turn were associated with lower levels of depression, somatic complaints, and blood cholesterol. Similarly, poor leadership has consistently been shown to have a negative impact on employee health (Kelloway, Sivanathan, Francis, \& Barling, 2005). Research by Lim, Cortina, and Magley (2008) demonstrated a connection between workplace incivility and health. Incivility had a direct negative impact on mental health, and those with mental health problems were more likely to suffer from poorer physical health. Addressing another form of mistreatment at work, Rospenda, Richman, Ehmke, and Zlatoper (2005) found longitudinal and cross-sectional results that general workplace harassment and sexual harassment were related to increased odds of illness. Research by Parkes (1999) additionally demonstrated that both job type and shift affected specific health outcomes.

Summary. As the research demonstrates, numerous variables affect levels of physical and mental health in employees. A tendency to contribute to stress-inducing situations can be considered a commonality among the predictors discussed. The predictor variable proposed in this research study, job demands, has similarly been demonstrated to be a stressor that leads to increased levels of health-affecting strain. The present dissertation aimed to demonstrate that the relationship between job demands and health crosses over between spouses. Further, a goal of the present study was to replicate findings that there is a crossover effect of health between spouses. 


\section{Job Demands and Health}

The psychological work environment, as a function of job demands, is the predictor of interest in the present study. As previously discussed, high job demands are linked to health within person (van der Doef \& Maes, 1999). The present dissertation study aimed to demonstrate how high demands can also affect the family system. It was proposed that this stressor will cross over to impact physical and mental health of the spouse.

Over the past 25 years, numerous research studies have tested the premises of Karasek's (1979) JDC(S) Model with respect to various outcomes. Next, research that connects the strain hypothesis to physical health of employees is addressed, followed by a review of research that relates high demand-low control situations to mental health. Following a review of research linking the strain hypothesis to physical and mental health, studies that have examined or found significant results for the direct effects of job demands individually are addressed.

Physical health. Van der Doef and Maes (1998) reviewed 51 studies that connect the JDC(S) model with physical health. Results suggest that working in a high (iso)strain job is associated with an elevated risk for cardiovascular disease, negative pregnancy outcomes, and increased psychosomatic complaints. Researchers have established a connection between the job strain condition (high demands and low control) and Cardiovascular Disease (CVD) or risk factors associated with CVD (Schall, Landsbergis, \& Baker, 1994). Theorell (2003) similarly found that high demands and low decision latitude is related to risk of developing CVD and gastrointestinal disorders. Belkic, Landsbergis, Schall and Baker (2004) found a strong and consistent association among 
men, although there was a sparse and less consistent connection between job strain and CVD risk for women. Demonstrating the importance of examining job demands and control together, Theorell and Karasek (1996) suggested that there have been few significant findings between blood pressure and demands or control as single factors.

Mental health. Research has examined the mental health outcomes of the job strain hypotheses as well. In a review of 63 empirical studies addressing the JDC(S) Model and psychological health, van der Doef and Maes (1999) found considerable support for the strain hypothesis. It was found that working in high strain jobs was associated with lower job satisfaction, more burnout and both lower general psychological well-being, as well as lower job-related psychological well-being. Sanne, Mykletun, Dahl, Moen and Tell (2005) similarly confirmed the strain hypothesis, finding that anxiety and depression levels increased linearly and considerably with increasing demands, iso-strain and strain scores and with decreasing social support and control scores. Further, demands and control were each independently associated with anxiety and depression levels. Research by Griffin, Greiner, Stansfeld, and Marmot (2007) compared the predictive validity of the JDC model, the Effort-Reward Imbalance model and the hindrance/utilization models on the mental health outcomes of anxiety and depression. Results showed that the JDC model accounted for the most variance associated with anxiety and depressive symptoms.

Physical and mental health. Numerous research studies have addressed the JDC(S) model by examining outcomes that include both physiological and psychological measures. In a human services population, de Jonge, Dollard, Dormann, Le Blanc and Houtman (2000) found that the combination of high demands and low control at work led 
to high levels of emotional exhaustion and psychosomatic health complaints. Research by Lerner et al. (1994) demonstrated that job strain was significantly related to numerous components of health-related quality of life, including physical functioning, role functioning related to physical health, vitality, social functioning, and mental health. Van der Doef, Maes, and Diekstra (2000) examined the JDC(S) model in relation to four occupational strain indicators. It was found that higher demands (time pressure), lower control (skill discretion, decision authority or task control), and lower support are associated with higher levels of psychosomatic complaints, more psychological distress, and more job dissatisfaction.

Nonsupportive studies addressing JDCS Model. As mentioned in the previous discussion of the JDC(S) model, not all research supports the hypotheses proposed by the model. Although research more consistently supports the strain hypotheses than the buffer hypothesis, nonsupportive studies do exist. For example, when studying a population of young, female nurses, Riese, Van Doornen, Houtman, and Geus (2000) found that in young healthy women, the combination of high demands and low control is not related to risk indicators for cardiovascular disease (CVD). In a review by van der Doef and Maes (1999) only 68\% of studies examining the relationship between job characteristics and psychological well-being supported the strain hypothesis.

Job demands and health. Some researchers have more specifically made the connection between job demands and health of employees. Fox, Dwyer and Ganster (1993), examining a population of nurses, found that objectively based job demands, patient load and percentage of patient contact time, were related to several physiological outcomes. Blood pressure both at work and after work, as well as after-work cortisol 
levels were significantly predicted by the objective measures of job demands. Research by Parkes, Mendham and Von Rabenau (1994) found that job demands were significantly predictive of psychosomatic complaints. Further, Stansfeld, Bosma, Hemingway and Marmot (1998) examined the effects of effort-reward imbalance, decision latitude, psychological demands, confiding and emotional support on health functioning in a sample of British civil servants. Results showed that poor physical and psychological functioning among women was best predicted by psychological demands at work.

Summary. Summing up the connection between the JDC model and overall health of employees, reviews of the relevant research suggest that there is good evidence for the main effects of job demands on job strain and various health outcomes (de Lange et al., 2003). Conversely, there is only modest support for the moderating influence of control, or any other interactive effects (e.g. support as a moderator). Based on these findings, the present study is interested in the main effects of job demands on the physical and mental health component scores of overall health. Extensive outcomes of negative health have been identified, including healthcare costs, turnover, absenteeism, poor morale, job satisfaction, negative health behaviors, CVD, organizational commitment, job performance, and organizational citizenship behaviors (Beehr \& Glazer, 2005; Quick et al., 1998). Considering the wide reaching effects employee health has on not only the quality of life of employees, but also on the productivity and functioning of the organization, job demands at work is an important variable to study in this context.

Although demands are related to health, there is a gap in the literature regarding how one partner's demands affect the other partner's health. It is proposed that levels of job demands will cross over to affect the mental and physical health of the spouse. This is 
a direct crossover argument. The definition of direct crossover suggests that the spouse takes on the stresses of his or her partner. Direct crossover can occur when the stress of one partner causes an empathetic reaction, increasing the level of stress or strain in the other partner. The spouse may experience those stressors like they are his or her own. Another possibility is that a high strain situation for an individual might put more pressure on his or her spouse, affecting the spouse's health. This could manifest in a variety of ways. For example, the high strain affecting the individual might decrease the amount of time he or she can contribute to household chores or taking care of dependents. This decrease in activity would affect the family system. One spouse may have to take on more responsibilities to make up for the strain affecting the other spouse, which may lead to increased stress, strain or unhealthy behaviors. Therefore, it follows that an employee's job demand levels will not only affect his or her own health, but also affect the health of the spouse. Another workplace variable that has been shown to affect health of employees is economic stress (Probst, 2004). The following section will address the role of economic stress, operationalized by income adequacy, as a potential common stressor mechanism in the crossover process between partners. 


\section{Chapter V}

\section{Income Adequacy as a Common Stressor}

The present study examines perceived income adequacy as a potential common stressor affecting health of both partners. Researchers have captured the idea of income adequacy in terms of one's perceived ability to meet demands. Westman and Vinokur (1998) suggested that common stressors that increase both partners' strain need to be considered spurious crossover. The common stressors shared in the environment of both partners may affect their strain levels. The increase in both partners' strain may then result in a positive correlation of the partners' strain, which may be erroneously interpreted as a genuine crossover effect. In other words, the common stressor can be viewed as a third variable that simultaneously increases the strain of each spouse (Westman et al., 2004b).

However, several studies have found evidence for the coexistence of the three crossover mechanisms (Howe et al., 2004; Westman \& Vinokur, 1998). Common stressors may affect each partner's strain and still cause crossover through other crossover mechanisms. Thus, the present dissertation study hypothesizes that the common stressor crossover mechanism and the direct crossover mechanism will cooccur. As Westman, Vinokur, Hamilton, and Roziner (2004) stated, "These three mechanisms of crossover can operate independently of one another and are not mutually exclusive. Therefore, it is quite possible that some of the proposed mechanisms operate jointly (pp. 771).”

In this case, the common stressors mechanism implies that crossover of health may be an outcome of a common stressor affecting the strain of both partners. The 
present study relies on a single item indicator of income adequacy developed by Stewart and Archbold (1996) asking participants "Which of the following four statements describes your ability to get along on your income?" Response options include: "we can't make ends meet"; "we have just enough, no more"; "we have enough, with a little extra sometimes"; and "we always have money left over".

\section{Financial Variables and Health}

Based on research that suggests a connection between financial strain and health, it is proposed that the measure of perceived income adequacy may be a common stressor that affects the health of each partner. Sears (2008) discussed several pathways through which financial stress can impact health of employees. First, money is critical for meeting basic survival needs. Income helps individuals afford basic necessities (i.e. food, shelter and water) as well as the necessities that enable individuals to work (i.e. childcare, transportation, and clothing). Second, money often serves a symbolic purpose in individuals' lives. For example, perceptions of income inadequacy may lead to a lower sense of self-worth and feelings of control over life (Pearlin, Lieberman, Menaghan \& Mullan 1981; Price, Choi \& Vinokur, 2002). Third, the financial situation may become a stressor itself. If worries about finances become draining, individuals are left with fewer psychological resources to allocate toward other life demands.

Various operationalizations of financial stress have been connected to physiological, and with greater frequency, psychological health of employees. Research by Olivius, Ostergren, Hanson and Lyttkens (2004) found that economic stress among parents was associated with low self-rated physical and mental health, even when controlling for employment status (i.e. considering involuntary unemployment). Positive 
mental health was linked to financial strain, as well as numerous other variables like a lack of physical health problems, age and being employed (Hu, Stewart-Brown, Twigg, \& Weich, 2007). Examining married couples, Mills, Grasmick, Morgan, and Wenk (1992) found a significant effect of economic strain on psychological well-being among the working husbands, as well as working wives. Economic strain also significantly reduced psychological well-being among wives that were not employed full time. Research by Peirce, Frone, Russell and Cooper (1994) also connected financial strain and depression, but further found depression was a mediator in the relationship between financial strain and drinking alcohol to cope. Several researchers addressed the relationship between financial strain and health in unemployed populations (McKeeRyan, Song, Wanberg, \& Kinicki, 2005; Price, Choi, and Vinokur, 2002).

Researchers have also demonstrated a connection between other financial variables and health. Research by Franks, Gold and Fiscella (2003) found that lower socioeconomic status was related to lower reported health status and higher mortality. Similarly, Liang et al. (1999) found a connection between dissatisfaction with one's financial situation and health. The Working Poor Families Project reports that one in four American working families now earn wages so low that they have difficulty surviving financially.

\section{Relevance in the Grocery Industry}

One study conducted in 1999, found that the grocery industry has undergone many changes in the past several decades (Hughes, 1999). This industry once was known to provide full-time, well-paid jobs, while the majority of grocery workers now hold parttime, low-wage positions. These changes are due to increased competition and 
deunionization within the industry (Hughes, 1999). The particular case study presented by the author demonstrated that entry-level wages had eroded and the use of part-time workers had increased.

Statistics found in the National Industry-Specific Occupational Employment and Wage Estimates (Bureau of Labor Statistics, 2008) demonstrate the current state of the grocery industry. When considering all occupations within the grocery industry, the mean annual salary is $\$ 24,390$, with a median hourly rate of $\$ 9.49$ and a mean hourly rate of $\$ 11.72$. This category of 'grocery stores' includes occupational titles that may be employed in the industry but are not represented in the present study's sample (e.g. accountants, PR specialists, security guards and HR specialists).

Several occupational titles included in the BLS statistics are the most indicative of employees in the present study sample. For example, a cashier is a common occupational title included in the present study. According to the BLS (2008), cashiers earn a mean annual salary of $\$ 19,780$, with a median hourly rate of $\$ 8.59$ and a mean hourly rate of \$9.51. Other positions representative of the study's population were included in the BLS statistics. For example, stock clerks earn a mean annual salary of $\$ 21,790$, the annual salary of pharmacy aides averages around $\$ 22,240$, and the mean annual salary of bakers is $\$ 24,990$. The poverty threshold in 2009 is $\$ 22,050$ for a family of four or $\$ 10,830$ for an individual (Department of Health and Human Services, 2009). Considering the present study's population of low-wage employees, it is appropriate to examine the role of financially rooted stressors as a potential common stressor mechanism in the crossover of health between partners. 


\section{Statistically Addressing Common Stressors}

There does not appear to be a consensus on how to statistically determine whether common stressors are the cause of crossover between partners. Some researchers simply treated the proposed common stressors as control variables. Bakker, et al. (2005) controlled for several demands and resources at home in order to attempt to rule out common stressors and resources as a spurious cause of crossover. Similarly, Haines et al. (2006) controlled for some common stressors that have been found to be linked to their outcome of interest, psychological distress. They claimed to rule out the common stressors mechanism by inclusion of these control variables.

Westman et al. (2004a) investigated the common stressors mechanism using a different approach than controlling for the common stressor variable, economic hardship. The researchers conducted hierarchical regressions including economic hardship, as well as excluding economic hardship. Comparing the two regressions, it was found that the significant crossover of anxiety between partners increased when economic hardship was not included in the equation. The $\Delta \mathrm{R}^{2}$ was significant, leading to the conclusion that there was a co-occurrence of both the direct crossover mechanism and the common stressors mechanism.

In order to rule out common stressors, Westman et al. (2001) employed a similar strategy. The researchers reanalyzed their data for a revised model that removed the effects of the spouse's job insecurity, the proposed common stressor, on the outcome of burnout. Instead they replaced the path from job insecurity to burnout with a correlation between the spouse's job insecurity and the residual of the spouse's burnout. They found that when the effects of their common stressor, job insecurity, are included in the model, 
there is not an increase in the direct crossover effect of the outcome of burnout in either direction.

Howe et al. (2004) addressed the common stressor mechanism by testing for differences between partners on the common stressors. A repeated measures analysis of variance (ANOVA) was conducted with results demonstrating that partners did not differ on the common stressor variables of life events and negativity. Next they tested for the amount of within-couple concordance on the common stressors. Correlational analyses found some support for the common stressor mechanism of crossover demonstrating that partners experienced the outcome.

Westman et al. (2008) tested the common stressor mechanism of crossover by simply examining the significance of the path between the common stressors and the outcome of perceived health. The authors used SEM to test their model, finding good fit. They supported the common stressors mechanism by demonstrating that economic hardship, one of the proposed common stressors, was significantly related to poor perceived health among both partners. The present dissertation will address common stressors using the same method as Westman et al. (2008). The present dissertation will also be using SEM to address model fit and examine the individual hypothesized relationships between variables. The following section will address the hypotheses, study method and proposed analyses in more detail. 


\section{Chapter VI}

\section{Present Study}

This dissertation study aimed to examine the linkages between perceived job demands of one working spouse and the health outcomes of the other spouse, as well as the direct crossover of health between spouses. Further, the role of perceived income inadequacy as a common stressor affecting health was addressed. Another important contribution of the present study is the proposed typology of crossover studies. The crossover hypotheses tested in the present study's models contribute to this typology, demonstrating its importance as a future organizing structure for crossover research. The present study examined the effects of demands on general health in 90 dyads from data collected for a study related to the national Work, Family, and Health Network led by Leslie B. Hammer, Ph.D. and Ellen Ernst Kossek, Ph.D. Data were collected in 12 stores of a grocery store chain in the Midwestern United States from an employee population, as well as a subset of employees' spouses. Employee data were collected via employee interviews given by myself and other research assistants. Participants from the Wave I Employee dataset who had a partner participate in the data collection were selected. These participants were matched with their partners from the Wave I Spouse dataset. The data was organized by dyads. All variables were named to denote gender. For example, for each dyad there is an age variable for the male partner (m_age) and an age variable for the female partner (f_age).

\section{Hypotheses}

Actor-Partner Interdependence Models. The two APIMs hypothesized in this study each examined within-person effects and direct crossover effects. Although the 
models hypothesized similar relationships between variables, the hypotheses and results for each model are discussed separately to simplify the discussion. The main purpose of analyzing the proposed models was to test new crossover hypotheses that will contribute to the literature. Many of the proposed relationships in the models are based on the requirements of developing an APIM, which provided the appropriate statistical techniques for measuring and testing interdependence. All relationships in the models, including those that replicate past research findings, are discussed. Physical Health was the dependent variable for the first model, while Mental Health was examined as the outcome of interest in the second model. This differentiation was necessary based on the finding that the variables of interest in the proposed models related differently to physical health and mental health composite scores. Some hypotheses in the second model are replications of hypotheses in the first model. It is necessary to include these hypotheses in both models based on the premise that similar hypotheses may have different results depending on the outcome variable included in the APIM.

Model 1. The first model (see figure 1) hypothesized that Job Demands, as measured by the Psychological Demands subscale of the Job Strain scale, predict a physical health composite score within person. It was further hypothesized that Job Demands of one spouse predict Physical Health for the other spouse (i.e. the first important crossover hypothesis). Income Adequacy was hypothesized to predict Physical Health within person. The exogenous variables (Men's Job Demands, Women's Job Demands, Men's Income Adequacy and Women’s Income Adequacy) were all expected to be correlated. Physical Health and Income Adequacy were also hypothesized to be correlated between spouses. The correlation of physical health is a second important 
crossover hypothesis in this model. This model addresses relationships that fall into three of the categories discussed in the typology of crossover studies proposed, including crossover of stressors between partners (stressor $\rightarrow$ stressor), stressors of one individual affecting the partner's strain (stressor $\rightarrow$ strain) and crossover of strain between partners (strain $\rightarrow$ strain). The within-person hypotheses for the first model are described first, followed by a description of the direct crossover hypotheses.

Within-person hypotheses. Several within-person effects were hypothesized for the first model. It was expected that Job Demands would predict levels of Physical Health. This hypothesis is a replication based on the strong connection between job demands and various measures of well-being in the literature (van der Doef \& Maes, 1999).

Hypothesis 1: Job demands will have a negative relationship with physical health.

In order to rule out common stressors, the relationship between income adequacy and physical health was examined for each member of the dyad. This hypothesis is not a replication but is based on supportive research findings that economic hardship serves as a common stressor affecting health (Westman et al., 2008).

Hypothesis 2: Income adequacy will have a positive relationship with physical health.

Direct crossover effects. Direct crossover effects are addressed by examining the relationships between the variables across partners. Crossover of job demands on physical health between partners is examined in the first model. In the proposed typology, this relationship would be categorized as a crossover of one individual's 
stressors affecting the partner's strain (stressor $\rightarrow$ strain). This hypothesis is not a replication and is based on research findings demonstrating crossover between different operationalizations of workplace stress and health. A majority of researchers have examined the crossover of work demands by investigating how the stress resulting from one individual's job affects the partner (for examples see Bolger et al., 1989; Crossfield et al., 2005; Dikkers et al., 2007; Jackson \& Maslach, 1982; Jones \& Fletcher, 1993a and 1993b; Lavee \& Ben-Ari, 2007; Long \& Voges, 1987; Morrison \& Clements, 1997; Pavett, 1986; Rook et al., 1981; Westman \& Etzion, 1999). Crossover of stress specifically on physical health has been demonstrated in the crossover literature (Eckenrode \& Gore, 1981; Fletcher, 1988; Gorgievski-Duijvesteijn et al., 2000; Jones and Fletcher, 1993b).

Hypothesis 3: Job Demands of one spouse will have a negative relationship with physical health of the other spouse.

Independent (exogenous) variables. Several of the proposed hypotheses are necessary to include based on the methods discussed in the upcoming section. This discussion will include an explanation of the Actor-Partner Interdependence Model (APIM); which is important to use in this case based on the fact that this type of analysis takes into account the interdependence of dyads. When using APIM it is necessary to consider correlations between the independent variables and correlations between residual variables (Cook \& Kenny, 2005). First, there is an important statistical role for the correlation between the exogenous, or independent variables (which is indicated by the curved, doubleheaded arrow). In the present study, it was hypothesized that all the 
exogenous variables would be correlated. The direct crossover of job demands between partners was examined. Additionally, the direct crossover of income adequacy between partners was examined. Both these relationships are considered a crossover of stressors between partners (stressor $\rightarrow$ stressor). These correlations ensure that if any of the exogenous variables predicts an endogenous variable, it is done while controlling for the other exogenous variables. Thus, actor effects are estimated controlling for partner effects, and partner effects are estimated controlling for actor effects.

Hypothesis 4: Job demands of spouses will be correlated.

Hypothesis 5: Income adequacy of spouses will be correlated.

Hypothesis 6: Job demands will be correlated with income adequacy within spouse.

Hypothesis 7: Job demands of one spouse will be correlated with income adequacy of the other spouse.

Dependent (endogenous) variables. Specification of a correlation between the residuals, or endogenous variables, controls for additional sources of nonindependence (Cook \& Kenny, 2005). This study hypothesized that the endogenous variables in the first model (e.g. physical health of both partners) will be correlated. The direct crossover of physical health between partners is examined, which is categorized as a crossover of strain between partners (strain $\rightarrow$ strain) in the proposed typology of crossover research. This hypothesis is a replication of previous findings demonstrating crossover of health between dyads. Providing support for the hypothesized direct crossover of strain between partners, researchers have demonstrated direct crossover of physical and mental health 
(Cronkite \& Moos, 1984; Dikkers et al., 2007; Gorgievski-Duijvesteijn et al., 2000; Katz et al., 1999; Vinokur et al., 1996; and Westman \& Vinokur, 1998).

Hypothesis 8: Physical health of spouses will be correlated.

Model 2. The second model (see figure 2) hypothesized that Job Demands predict Mental Health within person. It was further hypothesized that Job Demands of one spouse predict Mental Health for the other spouse (i.e. the first important crossover hypothesis). Income Adequacy is hypothesized to predict Mental Health within person. The exogenous variables (Men's Job Demands, Women's Job Demands, Men's Income Adequacy and Women's Income Adequacy) were all expected to be correlated. Mental Health and Income Adequacy were also hypothesized to be correlated between spouses. The correlation of mental health is a second important crossover hypothesis in this model. This model also examined relationships that fall into three of the categories discussed in the typology of crossover research (stressor $\rightarrow$ stress; stressor $\rightarrow$ strain; and strain $\rightarrow$ strain). The within-person hypotheses for the second model will be described first, followed by a description of the direct crossover hypotheses.

Within-person hypotheses. Several within-person effects are hypothesized for the first model. It was expected that Job Demands would predict Mental Health levels. This hypothesis is a replication based on the strong connection between job demands and various measures of well-being in the literature (van der Doef \& Maes, 1999).

Hypothesis 9: Job demands will have a negative relationship with mental health.

In order to rule out common stressors, the relationship between income adequacy and mental health is examined for each member of the dyad. This hypothesis is not a 
replication but is based on research showing economic hardship as a common stressor affecting health (Westman et al., 2008).

Hypothesis 10: Income adequacy will have a positive relationship with mental health.

Direct crossover effects. The direct crossover effects of job demands on mental health between partners was examined in the second model. In the proposed typology of crossover research this relationship is categorized as the crossover of one individual's stressors on the partner's strain (stressor $\rightarrow$ strain). This hypothesis is not a replication and was based on research findings demonstrating crossover between different operationalizations of workplace stress and health. A majority of researchers have examined the crossover of work demands by investigating how the stress resulting from one individual's job affects the partner (for examples see Bolger et al., 1989; Crossfield et al., 2005; Dikkers et al., 2007; Jackson \& Maslach, 1982; Jones \& Fletcher, 1993a and 1993b; Lavee \& Ben-Ari, 2007; Long \& Voges, 1987; Morrison \& Clements, 1997; Pavett, 1986; Rook et al., 1981; Westman \& Etzion, 1999). Crossover of stress specifically on mental health has been established in the crossover literature (Crossfield et al., 2005; Eckenrode \& Gore, 1981; Gareis et al., 2003; Hammer et al., 2005a; Howe et al., 2004; Jones \& Fletcher, 1993a and 1993b; Mitchell et al., 1983; Rook et al., 1991). Hypothesis 11: Job demands of one spouse will have a negative relationship with mental health of the other spouse.

Independent (exogenous) variables. When using APIMs, it is necessary to consider correlations between the exogenous variables, which are indicated by the 
curved, doubleheaded arrow (Cook \& Kenny, 2005). It was hypothesized that all the exogenous variables would be correlated (e.g. job demands and income adequacy). Each of these relationships can be categorized as crossover of stressors between partners (stressor $\rightarrow$ stressor) in the proposed crossover research typology. These correlations ensure that if any of the exogenous variables predict an endogenous variable, it is done while controlling for the other exogenous variables. Thus, actor effects are estimated controlling for partner effects, and partner effects are estimated controlling for actor effects.

Hypothesis 12: Job demands of spouses will be correlated.

Hypothesis 13: Income adequacy of spouses will be correlated.

Hypothesis 14: Job demands will be correlated with income adequacy within spouse.

Hypothesis 15: Job demands of one spouse will be correlated with income adequacy of the other spouse.

Dependent (endogenous) variables. Specification of a correlation between the residuals, or endogenous variables, controls for additional sources of nonindependence (Cook \& Kenny, 2005). It was hypothesized that the endogenous variables in the second model (e.g. mental health of both partners) would be correlated. The direct crossover of mental health between partners was examined, which can be categorized as a crossover of strain between partners (strain $\rightarrow$ strain) in the proposed typology of crossover research. This hypothesis was a replication of previous findings demonstrating crossover of health between dyads. Providing support for the hypothesized direct crossover of strain between 
partners, researchers have demonstrated direct crossover of physical and mental health (Cronkite \& Moos, 1984; Dikkers et al., 2007; Gorgievski-Duijvesteijn et al., 2000; Katz et al., 1999; Vinokur et al., 1996; and Westman \& Vinokur, 1998).

Hypothesis 16: Mental health of spouses will be correlated. 


\section{Chapter VII}

\section{Method}

\section{Participants and Procedures}

This study was part of a larger study related to the national Work, Family, and Health Network and was led by Leslie B. Hammer, Ph.D. and Ellen Ernst Kossek, Ph.D. The data are treated as archival. Data were collected in 12 stores of a grocery store chain in the Midwestern United States in October 2006. The number of employees per store ranged from 30-90. Participants in the employee dataset were current employees of the grocery chain that had been employed for a minimum of two months prior to the survey. A total of 360 workers participated in the survey. Workers' spouses or partners were also invited to participate in an almost identical survey for a total sample of 90 spouses. Participants from the employee dataset were matched with their partners from the spouse dataset, comprising a total of 90 dyads. The store employees were primarily female (86\%), Caucasian (88\%) and they had an average age of 36. Based on the need for the present study to examine dyads, only data for the 90 store employees that also had a spouse participate in the survey will be used in this study.

Participants were recruited by members of the Portland State University research team led by Leslie Hammer, Ph.D. and members of the Michigan State research team led by Ellen Kossek, Ph.D. with full support of the corporate Human Resources Department. Before researchers entered the stores, a notice was sent out by corporate notifying the store directors of the project. The research team members entered the stores and offered employees the opportunity to participate. It was stressed to the participants that the research project was not sponsored by their employer and that confidentiality would be 
strictly maintained. Store employees were offered a \$25 incentive if they agreed to participate. Store employees were also invited to have their employed spouses participate in an alternate form of the survey. Spouse surveys were sent home with the store employee and included a self-addressed, stamped envelope. Spouses also received a \$25 incentive.

First, participants filled out informed consent forms. All store employee participants were read the informed consent form, as well as given an additional copy for their records. The spouse participants were given two copies of the informed consent form. One copy was signed and returned with the survey in the envelope. The second copy was given to spouses for their records.

Second, surveys were administered individually in a face-to-face interview style to all employees with interviews lasting between 35-50 minutes on average. The surveys were completed on company time and were completely voluntary (see Appendix B for a version of the survey with scales delineated). The interview included approximately 196 survey-type questions, including some demographic questions as well as questions on work-family, safety, and health. Participants were informed that they were not required in any way by the company to fill out the survey and that they could withdraw their participation at any time. Biodata health measures were additionally collected from a subset of the population.

\section{Measures}

Job demands. Job demands refer to work stressors or workload (van der Doef \& Maes, 1999). Perceived job demands were measured using the psychological demands subscale of the Job Content Questionnaire developed by Karasek (1985) (see Appendix 
B). Five items measure job demands $(\alpha=.70)$. Responses ranged from 1 (strongly disagree) to 4 (strongly agree). A sample item for the psychological demands measure is: I have enough time to get the job done.

Job control. Job control refers to the ability to control work activities, and is primarily measured by assessing decision latitude (Karasek, 1979). Decision latitude consists of two components: decision authority and skill discretion (van der Doef \& Maes, 1999). Perceived job control was measured using the decision authority and skill discretion subscales of the Job Content Questionnaire developed by Karasek (1985) (see Appendix B). Three items measure the subscale of decision authority $(\alpha=.69)$. Six items measure the subscale of skill discretion $(\alpha=.71)$. Based on reliability analyses, one item was removed from the skill discretion subscale, leaving five items in the scale. Responses ranged from 1 (strongly disagree) to 4 (strongly agree) for both subscales. A sample item for the decision authority measure is: I have a lot of say about what happens on my job. A sample item for the skill discretion measure is: I get to do a variety of things on my job.

Supervisor support. Supervisor support was measured using the Family Supportive Supervisory Behavior scale developed by Hammer, Kossek, Yragui, Bodner, and Hanson (2009) (see Appendix B). Five items measured Emotional Support $(\alpha=.91)$. Three items measured Role Model Support $(\alpha=.75)$. Three items measured Instrumental Support $(\alpha=.86)$. Two items measure Creative Management $(\alpha=.89)$. Responses range from 1 (strongly disagree) to 5 (strongly agree), with a greater score indicating greater perceptions of the supervisor managing family-supportive behaviors. A sample item from the Emotional Support subscale is: My supervisor is willing to listen to my problems in juggling work and nonwork life. A sample item from the Role Model subscale is: My 
supervisor is a good role model for work and nonwork balance. A sample item from the Instrumental subscale is: I can depend on my supervisor to help me with scheduling conflicts if I need it. A sample item from the Creative Management subscale is: $M y$ supervisor is able to find ways to meet both the needs of associates and the business.

Physical health. Physical health refers to the physiological well-being component of overall health. Physical Health was measured with the SF-12 (v2) seven-item physical composite score (Ware, Kosinski, \& Keller, 1996) (see Appendix B). Scores were reverse-coded such that higher levels of the construct indicated more positive health. The reliability for the Physical Health Composite Score of the SF-12 is .89, as reported in the SF-12 manual and as demonstrated in a variety of national samples. A sample item is: During the past 4 weeks, how much of the time have you had any of the following problems with your work or other regular activities as a result of your physical health?

Mental health. Mental health refers to the psychological well-being component of overall health. Mental Health was measured with the SF-12 (v2) seven-item physical composite score (Ware, Kosinski, \& Keller, 1996) (see Appendix B). Scores were reverse-coded such that higher levels of the construct indicated more positive health. The reliability for the Mental Health Composite Score of the SF-12 is .86, as reported in the SF-12 manual and as demonstrated in a variety of national samples. A sample item for the mental health measure is: How much of the time during the past four weeks have you felt downhearted and depressed?

Income adequacy. Perceived income adequacy refers to an individual's perceived ability to meet demands and is a component of financial stress (Sears, 2008). Income adequacy was measured using a single item indicator of income adequacy 
developed by Stewart and Archbold (1996) (see Appendix B) asking participants "Which of the following four statements describes your ability to get along on your income?" Response options include: "we can't make ends meet"; "we have just enough, no more"; "we have enough, with a little extra sometimes"; and "we always have money left over". A higher score indicated a greater ability to meet financial demands.

Control variables. Based on the connection to health outcomes, income adequacy is a control variable that was included (Westman et al., 2008). It served as a proposed common stressor in the model. Other control variables that work-family and health researchers often include based on their connection to health are hours worked, age, ethnicity and whether or not the participant smokes (Allen \& Armstrong, 2006; Elovainio et al., 2002; Hammer et al., 2005).

\section{Analyses}

In order to address crossover between spouses a data analytic method must be used that allows researchers to investigate issues of mutual influence. The Actor-Partner Interdependence Model (APIM) is a model of dyadic relationships that integrates a conceptual view of interdependence in two person relationships. APIM also provides the appropriate statistical techniques for measuring and testing interdependence (Cook \& Kenny, 2005). Kashy and Kenny (2000) suggest using APIM to help control for the nonindependence of data that naturally occurs in relationship dyads. According to Campbell and Kashy (2002), “this model suggests that a person's independent variable score affects both his or her own dependent variable score (known as the actor effect), and his or her partner's dependent variable score (known as the partner effect)" (p. 328). 
Dyadic data analysis. Kenny, Kashy and Cook (2006) attempted to clarify the language in dyadic data analysis by specifying the types of dyads, the types of dyadic variables, and the types of dyadic designs. The types of dyads can be either distinguishable or indistinguishable. Distinguishability exists when there is a meaningful factor that can be used to give order to the two individuals. In the present study, the dyads are distinguishable based on gender. There are three types of dyadic variables: betweendyads, within-dyads and mixed variables. Mixed variables will be used in the present study, in that variation exists both within the dyad and between dyads. Partners' scores will differ and some dyads will have higher average scores than others.

Additionally, there are several types of dyadic designs. In the one-with-many designs, each person is linked to multiple others, but these others are only linked to that one person. The Social Relations Model (SRM) design is an extension of the one-withmany designs, with the addition that each partner also interacts with or is rated by multiple individuals. This dissertation study utilizes the standard design of dyadic data analysis in which each person is a member of one, and only one, dyad. Within this design, there are three ways in which dyadic data sets can be structured. In the case of the individual structure, each member of the dyad is treated as a single unit. This structure has several disadvantages, including the fact that it ignores nonindependence and fails to allow for the influence that partner characteristics can have on the person. In pairwise structures there is one record for each individual but both partners' scores occur on each record as well. The dyad structure, in which there is a single unit for each dyad, will be used in the present study. 
Actor-Partner Interdependence Model. The Actor-Partner Interdependence Model (APIM) is used to estimate the effects of mixed independent variables, which was used in the present study (Kenny et al., 2006). There are two effects in the APIM: the effect of a causal variable on the person's own response, an actor effect, and the effect of a causal variable on the partner's response, a partner effect (Kenny et al., 2006). The two most central components of the APIM are the actor effects and partner effects (Cook \& Kenny, 2005).

There are two additional features of the APIM to consider: correlations between the independent variables and correlations between residual variables (Cook \& Kenny, 2005). First, there is an important statistical role for the correlation between the exogenous, or independent variables (which is indicated by the curved, double-headed arrow). In the present study, it was hypothesized that all the exogenous variables would be correlated. The correlation ensures that if any of the exogenous variables predicts an endogenous variable, it is done while controlling for the other exogenous variables. Thus, actor effects are estimated controlling for partner effects, and partner effects are estimated controlling for actor effects. Kenny et al. (2006) stated that researchers can control for independent variables by computing a partial correlation between the two dyad members' scores, partialing out the effects of the independent variables. One additional degree of freedom is lost for every variable that is controlled.

Second, specification of a correlation between the residuals, or endogenous variables, controls for additional sources of nonindependence (e.g. family effects). The present study hypothesizes that the endogenous variables (e.g. physical and mental health of both partners) will be correlated. Researchers may also include additional variables in 
the specified models, beyond the independent and residual variables, if there is a theoretical basis for their inclusion (Kenny et al., 2006). If the added variables measure characteristics of the individuals in the dyad, their effects on the dependent variables would also be either actor or partner effects. Based on this recommendation, the present study hypothesizes that the common stressor variable of income adequacy will have actor and partner effects.

Nonindependence. Kashy and Kenny (2000) stated that the APIM is used to help control for the non-independence of data that naturally occurs in relationship dyads. The presence of nonindependence is determined by measuring the association between the scores of the dyad members (Cook \& Kenny, 2005). Different measures are used depending on the type of dyad. For indistinguishable dyad members, like identical twins or same-sex couples, nonindependence is measured with the intraclass correlation. For dyads with distinguishable members, such as the present study, nonindependence can be measured with the Pearson product-moment correlation (Cook \& Kenny, 2005).

Structural equation modeling. Kenny et al. (2006) recommend using Structural Equation Modeling (SEM) to estimate the APIM for samples of distinguishable dyads. There are two main goals of an SEM analysis: to understand patterns of correlations among a set of variables and to explain as much of their variance as possible with the model specified by the researcher (Kline, 2005). Structural equation modeling with AMOS 7 was used to test the overall model fit and assess the hypothesized relationships.

There are several terms important to understand when modeling in SEM. First of all, there is a differentiation between indicators (i.e. observed variables) and latent variables (i.e. unobserved variables). A latent variable is not directly measured but is 
assessed indirectly by the measured variables. This differentiation is especially relevant for the measurement model in this study. Further, in SEM there is a differentiation of variables that is made in structural models. This categorization is valid for both latent and observed variables. Exogenous variables are independent variables and have no prior causal variable. They may be correlated with other exogenous variables as depicted by a double-headed arrow. Endogenous variables are either mediating variables or dependent variables. These variables are on the receiving end of single-headed arrows indicating a regression path. This path may originate from an exogenous variable or another endogenous variable. In the proposed models both exogenous and endogenous variables are at the origin of the paths ending with the endogenous variables.

Before conducting the SEM analyses, I measured nonindependence by correlating the dyad members' scores using a correlation coefficient (see Table 4). According to Myers (1979), a liberal test ( $\mathrm{p}<.20$, two-tailed) should be used in testing whether there is nonindependence since failure to detect nonindependence could lead to bias in significance tests. Nonindependence does not bias the effect estimates themselves, what are biased are the variances. Since variances are biased, standardized measures are also biased. Nonindependence always results in fewer degrees of freedom than there would be if the data were independent. If the independence of observations is supported statistically, then one could treat the individual rather than the dyad as the unit of analysis, doubling the sample size. However, very often the nonindependence is what is most interesting about dyadic data (Kenny et al., 2006).

Job demands were treated as observed variables in the structural model. The model is considered a nonrecursive path model as it includes feedback loops. Only 
observed variables were modeled and only the endogenous variables in the path models had error terms. The exogenous variables were assumed to be measured without error. Path models are similar to regression models because they only have observed variables, but using AMOS to specify the proposed path model has the benefit of measuring model fit.

For the SEM analysis, the first step in analyzing these relationships is to examine the overall fit of the proposed model. Due to the fact that there is no single significance test in SEM as there is in regression or analysis of variance (ANOVA), model fit was assessed by examining several fit indices. A nonsignificant $\chi^{2}$ indicates good fit. However, because $\chi^{2}$ is sensitive to sample size, good models may be inappropriately rejected (Ullman, 1996). Thus, several other fit indices have been created which assess model fit from various perspectives.

Several other fit indices have been created which assess model fit from differing perspectives. The RMSEA (root mean square error of approximation) test gives an estimate of the discrepancy between the model and population covariance matrices. RMSEA is a noncentrality-based index meaning it compares the model to the estimate of a best possible fit given the degrees of freedom in the model instead of comparing the model to one with perfect fit. To indicate good model fit I looked for an RMSEA value of less than .06 and not significant at the $p<.05$ level (Hu \& Bentler, 1999).

The NFI (normative fit index) was also assessed. Normative refers to the fact that the index varies from 0 to 1 , with 1 indicating perfect fit. This statistic gives the ratio of the hypothesized model to the independence model, indicating the degree to which the 
model fits the data better than the independence model (where it is assumed that there are no relationships between the variables). To indicate good model fit I looked for a NFI value of greater than .95 (Hu \& Bentler, 1999).

I also examined the CFI (comparative fit index), which is the same as the NFI except that it takes into account noncentrality. Comparative fit indices compare the tested model to an independent model, where the variables are completely unrelated to one another, and to a saturated model, where all the variables are perfectly related. Similarly, I looked for a CFI value of .95 or greater to indicate good model fit (Hu \& Bentler, 1999).

In summary, the structural model tested the full model. The path coefficients associated with each hypothesized relationship in the model were examined for their magnitude, expected direction, and statistical significance. I then used these path coefficients to assess the hypothesized relationships. 


\section{Chapter VIII}

\section{Results}

\section{Missing Data}

Although the data for this dissertation were collected via interviews resulting in virtually no missing data, there were still some important decisions to be made regarding the instances where missing data occurred. In these instances, the data were coded as missing using a -1 . When data were not present due to the fact that the question was not applicable to a certain individual, a -7 was coded to represent a skip pattern. Finally, missing data were coded as -2 when two responses were checked off for one item. When it was necessary to make important decisions with regards to coding of data, the project manager was consulted, and all decisions were reported in the associate and supervisor codebooks. These types of decisions included instances where interviewers recorded more than one response option or an incorrect response that was illogical. In addition, when calculating scales to be used in the current study analyses, a $66 \%$ rule was employed. Specifically, in order for a participant's score to be calculated for each scale,

they must have answered $66 \%$ of the questions within that scale. These participants were not removed from the dataset.

\section{Demographics}

Tables 1, 2 and 3 contain descriptive data for demographics for the APIMs. The final sample for these models consisted of 90 couples. Since each of the 90 cases contained data for one male and one female partner, demographics are discussed separately for men and women. Male participants were an average age of 43 years old and $91 \%$ were Caucasian. They worked an average of 40 hours per week and the mean 
number of children living at home for the men was 1.3. The largest grouping of male participants had a high school diploma or GED (41\%), and had an average household income between $\$ 25,000$ and $\$ 40,000$ per year (31\%). On average, female participants were 40 years old and $87 \%$ were Caucasian. Women worked an average of 32 hours per week and had a mean of 1.3 children living at home. The largest grouping of female participants had a high school diploma or GED (45\%), and had an average household income between $\$ 25,000$ and $\$ 40,000$ per year $(32 \%)$.

A set of post-hoc analyses addressing the JDCS Model was also included in the present study. Hierarchical multiple regression analyses were conducted using the employee population of grocery store employees (i.e. not including spouse data). There were 360 total participants in the employee population, with $72 \%$ being female. Ninetytwo percent of the population was Caucasian and the average age was 38 years old. They worked an average of 31 hours per week and had an average of less than 1 child living at home $($ mean $=.8)$. The largest percentage of participants had a high school diploma or GED (54\%), and had an average household income between $\$ 25,000$ and $\$ 40,000$ per year $(28 \%)$.

\section{Correlations}

Nonindependence. Intercorrelations for all variables used in the analyses are presented in Table 4. Nonindependence was tested using correlation coefficients of the dyad members' scores; these correlation coefficients are bolded in Table 4. All variables that were measured for both partners were significantly correlated between the men and the women. Correlations suggest a strong positive association between the measurements for men and women for job demands, income adequacy, physical health and mental 
health. These results demonstrate nonindependence of scores between partners, suggesting that the dyad should be treated as the unit of analyses rather than the individual.

Other correlations. Two differing variables were found to be correlated between partners; women's income adequacy and men's mental health had a strong positive association. There were also several correlational relationships found within partners. Men's mental health had a strong positive association with both men's job demands and men's income adequacy. Finally, women's physical health and mental health was found to have a moderate negative association.

\section{Hypothesis Testing}

Hypotheses 1 - 8. Two structural models, based on data from 90 dyads, were analyzed using AMOS 7. In the first model it was hypothesized that Job Demands (as measured by the Psychological Demands subscale of the Job Strain scale) would predict a physical health composite score within person. It was further hypothesized that Job Demands of one spouse would predict Physical Health for the other spouse. Income Adequacy was hypothesized to predict Physical Health within person. The exogenous variables (Men's Job Demands, Women's Job Demands, Men's Income Adequacy and Women's Income Adequacy) were all expected to be correlated. Physical Health and Income Adequacy were also expected to be correlated between spouses. Good model fit is evidenced by a non-significant chi-square, a CFI of at least .95, and a RMSEA of .05

or less (Kline, 1998). The structural model fit the data well, $\chi^{2}(2, \mathrm{~N}=90)=1.22, \mathrm{p}=.54$, $\mathrm{CFI}=1.0, \mathrm{RMSEA}=.00$. The model is very close to perfectly fitting the data. A just- 
identified fits the data perfectly well, meaning that there are as many known values as unknown values in the mode, resulting in zero degrees of freedom. The hypothesized model is not just-identified as it has two degrees of freedom. The fit indices fail to provide valuable information though, based on the close proximity of the model to being just-identified. The discussion of the results will focus on the value of the coefficient estimates.

Significant hypotheses. There was mixed support for the hypotheses in the first model (see Table 5). The significant hypotheses will first be discussed followed by a review of the nonsignificant hypotheses. Hypotheses 1 was partially supported, while hypotheses 4, 5 and 7 were fully supported. Addressing hypothesis 1, Men's Physical Health was significantly predicted by their own Job Demands (standardized coefficient $=$ -.25). Holding women's Job Demands, women's Income Adequacy and men's Income Adequacy constant, for every one standard deviation increase in men's Job Demands there was a corresponding .25 standard deviation decrease in the composite score for men's Physical Health. Hypothesis 1 is only partially supported; although this relationship was significant for the men, it was not a significant relationship for the women.

Several correlations between exogenous variables were found to be significant. Addressing hypothesis 4, men's and women's Job Demands were significantly correlated $\left(R^{2}=.25, p<.05\right)$. Similarly, hypothesis 5 was supported. Men's and women's Income Adequacy levels were significantly correlated $\left(R^{2}=.53, p=0\right)$. Finally, hypothesis 8 , addressing the correlation of endogenous variables, was supported. It was found that 
men's and women's Physical Health was significantly correlated $\left(R^{2}=.31, p<.01\right)$. This hypothesis was considered an important crossover hypothesis in the present study.

Nonsignificant hypotheses. The proposed crossover relationships, hypotheses 2 and 3, were both nonsignificant. In order to rule out common stressors, the relationship between income adequacy and physical health was examined for each member of the dyad (hypothesis 2). Income adequacy was not found to significantly predict physical health for either partner, ruling out income adequacy as a common stressor crossover mechanism. Hypothesis 3 addressed direct crossover between spouses, the other important crossover hypothesis for the present study. Men's Job Demands did not significantly predict women's physical health levels and Job Demands of women did not significantly predict men's physical health. Further, hypotheses 6 and 7 were not significant. Hypothesis 6 addressed the correlation between the exogenous variables of Job Demands and Income Adequacy within partners, while hypothesis 7 addressed this relationship between partners.

Hypotheses 9 - 16. The second model hypothesized that Job Demands would predict Mental Health within person, and that Job Demands of one spouse would predict Mental Health for the other spouse. Income Adequacy was hypothesized to predict Mental Health within person. The exogenous variables (Men's Job Demands, Women's Job Demands, Men's Income Adequacy and Women's Income Adequacy) were all expected to be correlated. Mental Health and Income Adequacy were also expected to be correlated between spouses. The structural model fit the data well, $\chi^{2}(2, \mathrm{~N}=90)=1.08$, $\mathrm{p}=.58, \mathrm{CFI}=1.0, \mathrm{RMSEA}=.00$. As mentioned previously, these fit statistics do not 
provide valuable information based on the close proximity of the model to a justidentified model.

Significant hypotheses. There was mixed support for the hypotheses in the second model (see Table 6). Hypotheses 8 and 9 were partially supported, while hypotheses 12, 13 and 16 were fully supported. Several within-person hypotheses were supported. Addressing hypothesis 9, men's Job Demands significantly predicted Mental Health for men (standardized coefficient $=.22$ ). Holding women's Job Demands, women's Income Adequacy and men's Income Adequacy constant, for every one standard deviation increase in men's Job Demands there was a corresponding .22 standard deviation increase in the composite score for men's Mental Health. Hypothesis 9 was only partially supported since the expected relationship was found to be significant only for males.

Hypothesis 10 was also partially supported. Mental Health for men was significantly predicted by men's Income Adequacy (standardized coefficient $=.34$ ). Holding women's Income Adequacy, men's Job Demands and women's Job Demands constant, for every one standard deviation increase in men's Income Adequacy there was a corresponding .34 standard deviation increase in the composite score for men's Mental Health. Although this association was found to be significant, the basis for this "common stressor' hypothesis is not supported. The common stressor of income adequacy only significantly predicts mental health within person for men. The common stressor hypothesis requires the relationship to be significant for both partners in order to assume that income adequacy is a common stressor simultaneously affecting mental health of men and women. 
Several correlations between exogenous variables were found to be significant. Addressing hypothesis 12, men's and women's Job Demands were significantly correlated $\left(R^{2}=.25, p<.05\right)$. Similarly, hypothesis 13 was supported. Men's and women's Income Adequacy levels were significantly correlated $\left(R^{2}=.53, p<.01\right)$. Finally, hypothesis 16, addressing the correlation of endogenous variables, was supported. Men's and women's Mental Health was significantly correlated $\left(R^{2}=.28, p<\right.$ $.01)$. This is considered an important crossover hypothesis for the present study.

Nonsignificant hypotheses. The proposed crossover relationships, hypotheses 10 and 11, were not fully supported. Hypothesis 10 was partially supported while hypothesis 11 was nonsignificant. In order to rule out common stressors, the relationship between income adequacy and physical health was examined for each member of the dyad (hypothesis 10). As mentioned in the significant hypotheses section, income adequacy was found to significantly predict physical health for men. Since this relationship was not significant for women, income adequacy as a common stressor crossover mechanism is ruled out. Hypothesis 10 addressed direct crossover between partners, the other important crossover relationship in this model. Men's Job Demands did not significantly predict women's physical health levels and Job Demands of women did not significantly predict men's physical health. Further, hypotheses 14 and 15 were not significant. Hypothesis 14 addressed the correlation between the exogenous variables of Job Demands and Income Adequacy within partners, while hypothesis 15 addressed this relationship between partners. 


\section{Summary of Results}

Tables 10 and 11 provide a summary of results for all hypotheses. Both hypothesized APIMs were found to have close to perfect fit. Within these models, some hypothesized relationships were supported while others were not found to be significant. Job Demands were found to significantly predict physical health (H1) and mental health (H9) within person for men. The only supported crossover hypotheses were correlational. Men's and women's income adequacy were significantly correlated (H5, H13). The construct of income adequacy has too much overlap between partners to be considered crossover. Men's and women's job demands were also significantly correlated (H4, H12), indicating a crossover of job demands between partners. Both men's and women's physical health (H8) and men's and women's mental health (H16) were significantly correlated. These findings were hypothesized based on the literature and can be interpreted as crossover of health between partners.

\section{Alternate Analyses}

Alternate APIM 1. The first alternative structural model, based on data from 90 dyads, was also performed through AMOS 7 (see Figure 3). In comparison to the hypothesized model, the first alternative model includes both Physical and Mental Health endogenous variables (instead of only including one health outcome). It was important to examine both physical health and mental health outcomes in one model based on literature that suggests psychological and physiological outcomes of stressors often occur together (Sonnetag \& Frese, 2003). Further, the following two alternate APIMs are trimmed, in order to determine which model is parsimonious yet fits the data reasonably well. Stansfeld, Bosma, Hemingway and Marmot (1998) examined the effects of effort- 
reward imbalance, decision latitude, psychological demands, confiding and emotional support on health functioning in a sample of British civil servants. Results showed that poor physical and psychological functioning among women was best predicted by psychological demands at work.

It was hypothesized that Job Demands would predict Physical Health within person. Job Demands were also expected to predict a mental health composite score within person. Income Adequacy was hypothesized to predict Physical Health and to predict Mental Health within person. It was further hypothesized that Job Demands of one partner would predict Physical Health for the other partner and that Job Demands of one partner would predict Mental Health for the other partner. Job Demands, Income Adequacy, Physical Health and Mental Health were expected to be correlated between partners. The structural model fit the data relatively well, $\chi^{2}(8, \mathrm{~N}=90)=10.83, \mathrm{p}=.21$, $\mathrm{CFI}=.95, \mathrm{RMSEA}=.06$.

Physical Health for the men was predicted by men's Job Demands (standardized coefficient $=-.23$ ). Men's Mental Health was predicted by men's Job Demands ( standardized coefficient $=.21)$. Men’s Physical Health was predicted by Income Adequacy for men (standardized coefficient $=.21$ ). Mental Health for men was predicted by men's Income Adequacy (standardized coefficient $=.34$ ). Men's and women's Job Demands were significantly correlated $\left(R^{2}=.25, p<.05\right)$. Men's and women's Income Adequacy was significantly correlated $\left(R^{2}=.53, p=.00\right)$. Men's and women's Physical Health was significantly correlated $\left(R^{2}=.31, p<.01\right)$. Finally, men's and women's Mental Health was significantly correlated $\left(R^{2}=.28, p<.05\right)$. 
Holding women's Job Demands, men's Income Adequacy and women's Income Adequacy constant, for every one standard deviation increase in men's Job Demands there was a corresponding .23 standard deviation decrease in the composite score for men's Physical Health. Holding women's Job Demands, men's Income Adequacy and women's Income Adequacy constant, for every one standard deviation increase in men's Job Demands there was a corresponding .21 standard deviation increase in the composite score for men's Mental Health. Holding women's Income Adequacy, men’s Job Demands and women's Job Demands constant, for every one standard deviation increase in men's Income Adequacy there was a corresponding .21 standard deviation increase in the composite score for men's Physical Health. Holding women's Income Adequacy, men's Job Demands and women's Job Demands constant, for every one standard deviation increase in men's Income Adequacy there was a corresponding .34 standard deviation increase in the composite score for men's Mental Health.

Alternate APIM 2. A second alternative structural model, based on data from 90 dyads, was additionally performed through AMOS 7 (see Figure 4). The model eliminates the common stressor variables (Income Adequacy for men and women) and includes both Physical and Mental Health endogenous variables. This alternate model is a trimmed version of the previous alternate model. Several alternate models were analyzed in order to explore which model is parsimonious yet fits the data reasonably well. This APIM was proposed to explore the relationships between study variables without income adequacy.

It was hypothesized that Job Demands would predict a physical health composite score within person. Job Demands were also expected to predict a mental health 
composite score within person. It was further hypothesized that Job Demands of one partner would predict Physical Health for the other partner and that Job Demands of one partner would predict Mental Health for the other partner. Job Demands, Physical Health and Mental Health were all expected to be correlated between partners. The structural model fit the data well, $\chi^{2}(2, \mathrm{~N}=90)=.95, \mathrm{p}=.62, \mathrm{CFI}=1.0, \mathrm{RMSEA}=.00$.

Physical Health for men was predicted by men's Job Demands (standardized coefficient $=-.22$ ). Men's Mental Health was predicted by Job Demands for the men (standardized coefficient $=.24)$. Men's and women's Job Demands were significantly correlated $\left(R^{2}=.25, p<.05\right)$. Physical Health for men and women was significantly correlated $\left(R^{2}=.29, p<.01\right)$, as was Mental Health $\left(R^{2}=.32, p<.01\right)$. Women's Physical Health and women's Mental Health was significantly correlated $\left(R^{2}=-.24, p<\right.$ .05). Holding women's Job Demands constant, for every one standard deviation increase in men's Job Demands there was a corresponding .22 standard deviation decrease in the composite score for men's Physical Health. Holding women's Job Demands constant, for every one standard deviation increase in men's Job Demands there was a corresponding .24 standard deviation increase in the composite score for men's Mental Health.

Alternate APIM 3. Next, a third alternative structural model, based on data from 90 dyads, was performed through AMOS 7 (see Figure 5). The model eliminates the exogenous variable Job Demands but includes both Physical and Mental Health as endogenous variables. This APIM explores the relationships between variables without the inclusion of job demands in the model. This alternate model is also a trimmed version of the first alternate model. Several different alternate models were analyzed in order to explore 
which model is parsimonious yet fits the data reasonably well. This APIM was proposed to explore the relationships between study variables without job demands.

It was hypothesized that Income Adequacy would predict Physical Health within person. Income Adequacy was also expected to predict Mental Health within person. It was further hypothesized that Income Adequacy of one partner would predict Physical Health for the other partner and that Income Adequacy of one partner would predict Mental Health for the other partner. Income Adequacy, Physical Health and Mental Health were all expected to be correlated between partners. The structural model fit the data well, $\chi^{2}(2, \mathrm{~N}=90)=1.82, \mathrm{p}=.40, \mathrm{CFI}=1.0, \mathrm{RMSEA}=.00$.

Men's Mental Health was predicted by Income Adequacy for the men (standardized coefficient $=.30$ ). Men's and women's Income Adequacy was significantly correlated $\left(R^{2}=.53, p=.00\right)$. Physical Health for men and women was significantly correlated $\left(R^{2}=.29, p<.01\right)$. Men's and women's Mental Health was also significantly correlated $\left(R^{2}=.30, p<.01\right)$. Physical and Mental Health for women were significantly correlated $\left(R^{2}=-.24, p<.05\right)$. Holding women's Income Adequacy constant, for every one standard deviation increase in men's Income Adequacy there was a corresponding .30 standard deviation increase in the composite score for men's Physical Health. Holding women's Income Adequacy constant, for every one standard deviation increase in men's Income Adequacy there was a corresponding .30 standard deviation increase in the composite score for men's Mental Health.

\section{Post-Hoc Analyses}

Post-Hoc APIM 1. Next, several post-hoc structural models were examined using a measure of job satisfaction. Based on data from 90 dyads, the analysis was performed 
through AMOS 7 (see Figure 6). This model replicates the first hypothesized APIM (see Figure 1) with the exception that it substitutes the exogenous variable Job Demands with a variable measuring Job Satisfaction. This APIM explored the relationships in the model using a resource as the predictor instead of a demand. In the proposed typology of crossover research, this model would be considered a crossover of resources on strain levels (resource $\rightarrow$ strain).

It was hypothesized that Job Satisfaction would predict Physical Health within person. It was further hypothesized that Job Satisfaction of one partner would predict Physical Health for the other partner. Income Adequacy was hypothesized to predict Physical Health within person. The exogenous variables (Men's Job Satisfaction, Women's Job Satisfaction, Men's Income Adequacy and Women's Income Adequacy) were all expected to be correlated. Physical Health and Income Adequacy were also expected to be correlated between partners. The structural model fit the data well, $\chi^{2}(2$, $\mathrm{N}=90)=.78, \mathrm{p}=.68, \mathrm{CFI}=1.0, \mathrm{RMSEA}=.00$.

Women's Physical Health was predicted by Job Satisfaction for the women (standardized coefficient $=.32$ ). Men's and women's Income Adequacy was significantly correlated $\left(R^{2}=.53, p<.01\right)$. Physical Health for men and women was significantly correlated $\left(R^{2}=.29, p<.01\right)$. Job Satisfaction and Income Adequacy for men were significantly correlated $\left(R^{2}=.27, p<.05\right)$. Further, Men's Job Satisfaction and Income Adequacy for women was significantly correlated $\left(R^{2}=.22, p<.05\right)$. Holding men's Job Satisfaction and Income Adequacy for men and women constant, for every one standard deviation increase in women's Job Satisfaction there was a corresponding .32 standard deviation increase in the composite score for women's Physical Health. Holding 
women's Income Adequacy constant, for every one standard deviation increase in men's Income Adequacy there was a corresponding .30 standard deviation increase in the composite score for men's Mental Health.

Post-Hoc APIM 2. A second post-hoc structural model was examined using a measure of job satisfaction. Based on data from 90 dyads, the analysis was performed through AMOS 7 (see Figure 7). This model replicates the second hypothesized APIM (see Figure 2) with the exception that it substitutes the exogenous variable Job Demands with a variable measuring Job Satisfaction. This APIM explored the relationships in the model using a resource as the predictor instead of a demand. In the proposed typology of crossover research, this model would be considered a crossover of resources on strain levels (resource $\rightarrow$ strain).

It was hypothesized that Job Satisfaction would predict Mental Health within person. It was further hypothesized that Job Satisfaction of one partner would predict Mental Health for the other partner. Income Adequacy was hypothesized to predict Mental Health within person. The exogenous variables (Men's Job Satisfaction, Women's Job Satisfaction, Men's Income Adequacy and Women's Income Adequacy) were all expected to be correlated. Mental Health and Income Adequacy were also expected to be correlated between partners. The structural model fit the data well, $\chi^{2}(2$, $\mathrm{N}=90)=1.42, \mathrm{p}=.49, \mathrm{CFI}=1.0, \mathrm{RMSEA}=.00$.

Men's Mental Health was predicted by Job Satisfaction for the women ( standardized coefficient $=.22$ ). This finding supports direct crossover of resources on strain between partners. Men's Mental Health was also significantly predicted by Job Satisfaction for the men (standardized coefficient $=.22$ ) and Income Adequacy for the 
men (standardized coefficient $=.35$ ). Men's and women's Income Adequacy was significantly correlated $\left(R^{2}=.53, p<.01\right)$. Similarly, Mental Health for men and women was significantly correlated $\left(R^{2}=.23, p<.05\right)$. Job Satisfaction and Income Adequacy for men were significantly correlated $\left(R^{2}=.27, p<.05\right)$. Further, Men's Job Satisfaction and Income Adequacy for women was significantly correlated $\left(R^{2}=.22, p<.05\right)$.

Holding men's Job Satisfaction and Income Adequacy for men and women constant, for every one standard deviation increase in women's Job Satisfaction there was a corresponding .22 standard deviation increase in the composite score for men's Physical Health. Holding women's Job Satisfaction and Income Adequacy for men and women constant, for every one standard deviation increase in men's Job Satisfaction there was a corresponding .22 standard deviation increase in the composite score for men's Physical Health. Holding men's and women's Job Satisfaction and women's Income Adequacy constant, for every one standard deviation increase in men's Income Adequacy there was a corresponding .35 standard deviation increase in the composite score for men's Mental Health.

Model comparisons. Even if the hypothesized model fits the data well, it is often appropriate to analyze alternate models in Structural Equation Modeling (Kline, 2005). As can be seen in the present study, there can be several competing models based on slightly different hypotheses that may explain the observed relationships equally well. If this is the case, then researchers can reject the competing models. If the competing models explain the data better then researchers should reject the hypothesized model. When differences in fit between models are subtle, it is appropriate to compare the models with regard to their fit by calculating a chi square difference test. 
The chi square difference test is computed as the difference of model chi-square for the larger model and a nested model, for one degree of freedom. To test relative fit of a nested model, the smaller chi square and its degrees of freedom are subtracted from the larger chi square and degrees of freedom. If the chi-square difference shows no significant difference between the unconstrained original model and the nested, constrained modified model, then the modification is accepted on parsimony grounds.

The goal is to find the most parsimonious model which is well-fitting by a selection of goodness of fit tests. None of the alternate Actor-Partner Interdependence Models examined were trimmed versions of the hypothesized APIM, since they include both physical and mental health in the same thus none are a more parsimonious version of the model. Since the hypothesized models have good fit and are the most parsimonious versions of the models, chi square difference tests do not need to be calculated to justify their use.

JDCS Model Post-Hoc Analyses. According to Karasek's (1979) Job DemandControl (JDC) Model, a combination of job demands and job control determine the psychological work environment. Some researchers have redefined the model with the addition of work-related social support. The JDCS Model is based on the finding that social support may modify the impact of demands on and off the job (Johnson \& Hall, 1988). In the iso-strain hypothesis of the JDCS Model, jobs characterized by high demands, low control, and low support (iso-strain) are considered to be the work situations most detrimental to employee health. There are mixed results on whether or not proposed interactions in the JDCS Model are supported, even high-quality studies provide modest support for the interactional effects (de Lange et al., 2003). 
This set of post-hoc analyses addressed the three-way interaction of job demands, decision latitude and supervisor support in predicting health among employees. These analyses examined whether Karasek's (1979) Job Demand-Control-Support Model applies to the expanded employee population that the current sample of dyads is derived from. To test for moderation, hierarchical multiple regression analyses were calculated. In order to test for a three-way interaction, a regression analysis that includes all three independent variables, all three pairs of two-way interaction terms, and the three-way interaction term, was conducted. Two sets of analyses were conducted; addressing the dependent variables of physical health (see Table 8) and mental health (see Table 9) separately.

Predicting physical health. The first step was to enter the main effects of a measure of job demands (Psychological Demands), a measure of job control (Decision Authority) and a measure of support at work (Supervisor Support). This analysis was not significant. Job Demands, Decision Authority, and Supervisor Support did not account for a significant proportion of the variance in physical health, $R^{2}=.02, F(3,360)=2.23$, $p=.09$. Further examination of the main effects revealed that Decision Authority significantly accounted for some unique variance in physical health $(\beta=.13, p<.05)$. For every one-unit increase in Decision Authority, there was a corresponding .13 unit increase in the physical health composite score.

Next, the higher order terms of those three work characteristics were included to control for the possible effects of curvilinear relationships, as recommended by Lubinksy and Humphreys (1990). The second step was to enter the two-way interactions proposed 
in the Job Demand-Control-Support Model. This analysis was not significant. Job Demands, Decision Authority, Supervisor Support, and the two-way interactions (Demands X Control, Demands X Support, Control X Support) did not account for a significant proportion of the variance in physical health, $R^{2}=.02, F(6,360)=1.22, \mathrm{p}=$ .30. Further examination of the main effects revealed that Decision Authority significantly accounted for some unique variance in physical health $(\beta=.13, p<.05)$. In order to test for a three-way interaction, the third step was to conduct a regression analysis that includes all three independent variables, all three pairs of twoway interaction terms, and the three-way interaction term proposed in the Job DemandControl-Support Model. This interaction was not significant. Job Demands, Decision Authority, Supervisor Support, the two-way interactions (Demands X Control, Demands X Support, Control X Support), and the three-way interaction (Demands X Control X Support) did not account for a significant proportion of the variance in physical health, $R^{2}$ $=.02, F(6,360)=1.22, \mathrm{p}=.30$. Further examination of the main effects revealed that Decision Authority significantly accounted for some unique variance in physical health $(\beta=.13, p<.05)$. Table 8 provides a summary of the results of the first post-hoc model addressing the JDCS Model.

Predicting mental health. The first step was to enter the main effects of Job Demands, Decision Authority and Supervisor Support. This analysis was not significant. Job Demands, Decision Authority, and Supervisor Support did not account for a significant proportion of the variance in mental health, $R^{2}=.01, F(3,355)=1.31, \mathrm{p}=$ 
.27. Further examination of the main effects revealed that no variables significantly accounted for unique variance in mental health.

The second step was to enter the two-way interactions proposed in the Job Demand-Control-Support Model. This analysis not significant. Job Demands, Decision Authority, Supervisor Support, and the two-way interactions (Demands X Control, Demands X Support, Control X Support) did not account for a significant proportion of the variance in mental health, $R^{2}=.03, F(6,355)=1.84, \mathrm{p}=.09$. Further examination of the main effects revealed that Job Demands significantly accounted for some unique variance in mental health $(\beta=.11, p<.05)$.

The third step taken was to conduct a regression analysis that includes all three independent variables, all three pairs of two-way interaction terms, and the three-way interaction term proposed in the Job Demand-Control-Support Model. This analysis was not significant. Job Demands, Decision Authority, Supervisor Support, the two-way interactions (Demands X Control, Demands X Support, Control X Support), and the three-way interaction (Demands X Control X Support) did not account for a significant proportion of the variance in mental health, $R^{2}=.03, F(6,355)=1.56, \mathrm{p}=.14$. Further examination of the main effects revealed that Decision Authority significantly accounted for some unique variance in mental health $(\beta=.131 p<.05)$. Table 9 provides a summary of the results of the second post-hoc model addressing the JDCS Model.

Summary. Three steps were taken to address the three-way interaction proposed in the Job Demand Control Support Model. None of the three steps were found to be significant when predicting physical health or when predicting mental health. Some main 
effects were found to be significant. In hypotheses $17 \mathrm{~A}-\mathrm{C}$, decision authority significantly accounted for some unique variance in physical health $(\beta=.13, p<.05)$. In hypotheses 18 B - C, Job Demands were found to significantly account for some unique variance in mental health $(\beta=.11, p<.05 ; \beta=.13, p<.05$, respectively). 


\section{Chapter IX}

\section{Discussion}

Considering the tenets Systems Theory, it is essential to address the broader impact that stressors and strains can have beyond the individual employee. It has been well established that job demands play a critical role in predicting the health and wellbeing of employees (van der Doef \& Maes, 1999). The first primary contribution of the present dissertation study is the exploration of whether this impact on health extends into the family system, the goal being to show how the characteristics of an individual's job (i.e. high demands) impact his or her partner (i.e. physical and mental health). Further, another primary contribution of the present study is the proposed typology of crossover research. This typology could potentially lead to better organization and understanding of future crossover research. The primary and secondary contributions discussed previously will be addressed as well as other results from the dissertation analyses.

Researchers have demonstrated the crossover of multiple work-related stressors and strain between partners, including burnout (Bakker et al., 2007; Demerouti et al., 2004; Westman et al., 2001), work-family conflict (Cinamon et al., 2007; Hammer et al., 1997) and general workplace stress (Crossfield et al., 2005; Jones \& Fletcher, 1993b; Lavee \& Ben-Ari, 2007). There is a gap in the literature regarding the role that job demands can have in the crossover process. Job characteristics, such as job demands, influence the health of the individual employee (Karasek, 1979; van der Doef \& Maes, 1999). The present study aimed to extend this line of research into examining the impact job characteristics can have on the family system, namely the partner or spouse of the employee. 
It is crucial to ensure that job design is appropriate to encourage general health, not only because of the costs associated with health problems for the employee (e.g. absenteeism, productivity, turnover), but also because of the potential health problems related to the spouse or partner that may affect the company (e.g. increased family-towork conflict, health insurance costs). Exploring the more complete picture of how work stressors affect the employee and his or her partner can help researchers and practitioners understand the far-reaching impact that job design can have. Further, the present study is the first crossover study to target a sample of low-wage workers. As of 2008, 61 percent of women and 56 percent of men holding wage and salaried positions were paid by the hour, and an even greater proportion of workers in low-paid jobs are hourly (BLS, 2009a). This statistic demonstrates how important it is to examine how crossover dynamics play out in a population of hourly, low-wage employees and their partners.

\section{Examining the Results of Present Study}

Two models were hypothesized in this dissertation. The two Actor-Partner Interdependence Models were analyzed using Structural Equation Modeling in AMOS 7 (see Figures 1 and 2). The primary purpose of the models was to examine how relationships cross over between spouses. Each model additionally addressed a variety of between-person relationships, as well as within-person relationships. Several alternative models were also examined in order to explore the relationships between variables and find the most parsimonious model while still meeting the standards required for ActorPartner Interdependence Models (see Figures 3, 4 and 5). The originally hypothesized Actor-Partner Interdependence Models (APIMs) were examined post-hoc, with the replacement of a resource as the predictor variable (job satisfaction) instead of a demand 
(job demands) (see Figures 6 and 7). Post-hoc analyses also included two models analyzed using hierarchical multiple regression to test the Job Demand-Control-Support Model in a comparable population of grocery store employees.

Discussion of key findings. The results suggest mixed support for the hypotheses. One of the important crossover hypotheses in this study, proposed crossover of one partner's job demands on the other partner's health, was not supported. As will be discussed later in this section, there are many possible explanations for a lack of significant direct crossover relationships. Although the direct crossover relationships were not supported, several other crossover relationships were found. There was significant crossover of health (both physical and mental) between partners, as well as a significant crossover relationship of job demands between partners. Examining the crossover of health was considered a secondary goal. This crossover relationship was not the main focus of the present study as it has already been demonstrated in the literature (Barnett et al., 1995; Dikkers et al., 2007; Katz et al., 1999; Westman et al., 2008; Westman \& Vinokur, 1998).

Unexpected findings. Many relationships discovered in the present study's results were not expected. Most of these unexpected findings were related to the female partners in the sample. For example, job demands for the women were not found to be significantly associated with any other study variables (i.e. income adequacy, physical health, mental health, or job satisfaction for either partner) with the exception of the same measure for their male partner (i.e. male's job demands). When considering the post-hoc analyses, a similar pattern emerged for a measure of job satisfaction for the women. Job satisfaction also did not significantly relate to any other study variable; although in this 
case, women's job satisfaction was also not associated with men's job satisfaction.

Women's income adequacy was not found to be significantly associated with any of the other variables measured for women, although it was associated with men's levels of mental health and job satisfaction. These associations were most likely based in the finding that men's and women's income adequacy was so strongly related. Finally, women's physical health and mental health were found to have a negative association (men's physical and mental health were not associated at all). All of the described relationships for women are contrary to established literature and previous findings.

The relationships concerning the measures for the men followed a more expected pattern, with one notable exception. It was found that men's job demands had a negative relationship with physical health, yet a positive relationship with mental health. It would be expected that job demands for men would have a negative impact on both composite scores addressing health.

Potential explanations. There are many possible ways to interpret the unexpected findings in the present study. A number of these possible arguments will be discussed, ranging from measures to the sample studied, followed by a discussion of the many ways this study contributes to the field.

Measures. To better understand the data used to assess the proposed models, a closer look must be taken at the study variables. First, examining the average level of job demands reported for both males and females showed that very few participants endorsed responses that "agreed" with the job demands items. Overall, neither males nor females had high average levels of job demands (2.36 and 2.34, respectively). The mid-level scores on the job demands scale could potentially play a role in the lack of significant 
crossover findings. It is possible that job demand levels were not high enough to cross over and affect the health outcomes of the spouse or partner. Further, Activation theory suggests that there is a curvilinear relationship between stressors and strain, in that exposure to levels of job demands that are too low or too high would be potentially unfavorable for health outcomes (Sutherland \& Cooper, 2000; Warr, 1987, Xie \& Johns, 1995). This concept could partially explain the lack of significant findings connecting female's job demands with health outcomes, although these within-person relationships were significant for men.

It was important to also examine the physical health and mental health composite scores based on some unexpected relationships between study variables and the health outcomes. For example, it was found that men had a significant positive relationship between job demands and mental health but a negative relationship between job demands and physical health. On a scale from 0 to 100 , with scores being calibrated so that 50 is the average score or norm, men had an average physical health composite score of 49.99 and an average score of 48.60 on the mental health composite.

The overall average of physical health and mental health is very similar but when closely examining the scores case by case, there is a lot of variance between-person. For example, some individuals have similar scores for physical and mental health (e.g. 55.86 and 54.79, respectively) while others have very big differences between scores (e.g. 64.83 and 17.61, respectively). Similar patterns were detected for female participants, who had an average of 50.15 physical health composite score and 49.45 mental health composite score. The latter pattern described (i.e. big differences between scores) may be more of a factor for females considering the negative relationship between physical and 
mental health. When screening the health outcome data for both men and women, only one outlier was found for women's physical health. Removing this case did not significantly change the results from the hypothesized model addressing physical health.

The appropriateness of the measures addressing health in the present study should also be considered. The SF-12 provides psychometrically-based physical component summary and mental component summary scores (Gill, Butterworth, Rogers \& MacKinnon, 2007). Instead of directly assessing symptoms, the items address the outcomes of having the health symptoms (i.e. how their health levels affect their everyday activities, etc.). The SF-12 is useful in assessing the health of general and specific populations, addressing the relative burden of diseases (Ware et al., 1996). The SF-12 is used widely because of its brevity balanced with the breadth of health information it provides regarding functioning due to physical and mental health (Gill et al., 2007). The physical and mental health composite scores provided by the SF-12 can be better described as addressing health functioning rather than specific symptoms of health. Although this has the benefit of being a more objective assessment of how health symptoms are affecting the individual, it could have differential effects on research findings. For example, individuals may differ in how their mental or physical health levels manifest into their activities or daily functioning.

Method. Although the data analytic method used in the present study is considered a contribution, there are some drawbacks to using Actor-Partner Interdependence Models to address the hypotheses. The use of APIMs is suggested to help control for the non-independence of data that naturally occurs in relationship dyads (Kashy \& Kenny, 2000). The two most central components of APIMs are the actor 
effects (i.e. effects of a causal variable on the person's own response) and partner effects (i.e. effects of a causal variable on the partner's response) (Cook \& Kenny, 2005). When addressing models, actor effects are estimated controlling for partner effects, and partner effects are estimated controlling for actor effects. This is necessary when studying couples but also provides more conservative estimates than the alternate method of analysis; conducting individual regression analyses to address each hypothesis. Null results could be affected by the decreased power resulting from including all actor and partner effects in the same model. More constraints lead to decreased variance and thus more conservative estimates of the hypothesized relationships (Cook \& Kenny, 2005).

Gender and grocery industry. Two major issues that could be contributing to the lack of significant results in the present study include gender and the grocery industry. Although the sample included an equal number of males and females, a majority of the female partners were grocery store employees (e.g. 73.3\%) while the male partners worked in a greater variety of occupations. This could have potentially led to occupation being a confounding factor. It is difficult to tease apart whether gender or occupation is the cause for a many of the study results (e.g. lack of significant within-person hypotheses for the women).

Gender. When examining the variance of the measures used in the present study, there was consistently higher variability in responses for men in comparison to women. The reason for the higher variability is most likely based on the fact that the men worked in a greater variety of occupations than the women, as discussed previously. The lower variability demonstrated in women's measures could be one of the factors accounting for the lack of significant results within-person for the women. 
Possible links to gender could be based in past research that has indicated that men and women do not place the same amount of importance on various characteristics of paid employment, although these findings have been disputed (Rowe \& Snizek, 1995). Further, many of the positions held by the grocery store employees could be considered a job and not necessarily a career. This is another possible explanation for the lack of a significant connection between job demands and health for women.

Another gender-related explanation for the lack of a significant connection between women's job demands and their health outcomes is social support. If the women working in the grocery stores had a lot of social support at work; it is possible that the social support buffered the negative effects of job demands on health (van der Doef \& Maes, 1999). Although social support or coworker support was not measured in the present study, this author's experience interviewing the employees leads to the belief that it is a possible third variable to consider. The construct of social support would most likely be considered a gender difference, (e.g. women provide or need more social support in the workplace); though it is also a possibility that social support is part of the culture of grocery stores. Once again, it is not possible to tease these two variables apart in the present study.

As discussed in the Crossover chapter, early researchers suggested that there were gender differences in crossover. Research conducted in the 1980s found that crossover occurred predominately in the direction from male to female partners (e.g. Bolger et al., 1989; Burke et al., 1980; Fletcher, 1983; Jackson \& Maslach, 1982; Jackson et al., 1985; Long \& Voges, 1987; Mitchell et al., 1983; Riley \& Eckenrode, 1986). Many current researchers debate these findings for several reasons. For example, wives were mostly 
examined as passive recipients of stressors and strain from their husbands. Researchers often did not assess or control for the female partner's job and life stress, some samples mixing populations of employed and non-working women. It is argued that these early studies cannot rule out the possibility that what appears as direct crossover of stress from husbands to wives is an outcome of wives' job or life stress or of common family stressors of life events affecting both partners (Westman, 2001).

Overall, although the current study did demonstrate gender differences based on within-person analyses, there were not major differences in crossover results based on gender. Direct crossover of job demands on health was not demonstrated in either direction: from male to female partner or vice versa. Further, the crossover of physical and mental health between partners was bidirectional.

Grocery industry. Since the grocery employees included in this study were hourly, low-wage workers, there are some additional key characteristics to be discussed. First, considering there is a shortage of research examining this type of low-wage service industry population, it follows that many of the studies supporting the hypothesized within-person and crossover relationships were examining a more professional or salaried population.

Although there are many stressors associated with working in the service industry, one positive difference is that these types of employees typically cannot bring their work physically home. In contrast, a professional population may be able to continue to work past designated working hours by bringing documents or a laptop home. It is possible that hourly employees may be able to segment work and home better or may feel less attachment to their work based on the nature of the job. These differences of low-wage 
and hourly workers could play a part in explaining the nonsignificant association between female partners' job demands and health outcomes or the lack of a significant direct crossover between the individual's job demands and health outcomes of the partner. If the work is not as salient, holds less importance, or is better segmented from home life; the result may be a weaker connection between job demands and health outcomes.

Second, since hourly workers are much less likely to have health insurance coverage paid at least in part by their employers than other employees (Families and Work Institute, 2006), the women in this population were more unlikely to have health insurance than their male partners. It is possible that this plays a role in the findings that job demands were connected to health outcomes within-person for men but this was not the case for the women. This may not be likely considering the similarity in health scores for males and females, as mentioned previously. The issue will be further discussed later in this section, along with other distinguishing characteristics of hourly or low-wage workers.

There is not an easy fix to this confounding issue of gender and occupation within crossover research. It would be difficult for researchers to find a population of male and female partners or spouses that all work within the same occupation or field. Some researchers examine crossover hypotheses in random samples of participants (i.e. without a focus on any one industry), which was not a possibility for the present study based on the use of archival data.

\section{Meaning of Post-Hoc Analyses}

Job satisfaction. Several post-hoc APIMs were examined using a measure of job satisfaction in place of job demands in the originally hypothesized models (see Figures 6 
and 7). At the correlational level, women's job satisfaction was not significantly associated with any other study variables (see Table 4). On the other hand, there was a significant association between the measurement of male partners' job satisfaction with men' job demands, income adequacy and mental health, as well as with income adequacy for the women. The purpose of examining the relationships with job satisfaction was to contrast the findings with the originally hypothesized models that included job demands.

The correlational findings are similar between job demands and job satisfaction. Neither women's job demands nor women's job satisfaction were significantly associated with either of the outcome variables, physical health or mental health. Men's job satisfaction had a significant and positive relationship with men's mental health but was not significantly associated with physical health. This is contrasted with the findings that men's job demands had a significant positive relationship with men's mental health but a significant negative relationship with men's physical health. The findings demonstrate that both men's job demands and job satisfaction have a significant positive association with men's mental health. It is expected that job satisfaction would be associated with health outcomes; as it has been well-established in the literature (Faragher, Cass \& Cooper, 2005). This suggests that the men in the current sample may find the level of job demands they experience to potentially be a satisfying aspect of their work. Considering the average level of men's job demands are not on the high-end of the scale, this could be a logical conclusion. It is also important to mention that results from the APIMs demonstrated a significant direct crossover of women's job satisfaction on men's mental health. This was a direct crossover of women's resources on male's strain levels (resource $\rightarrow$ strain). 
JDCS Model. To better understand how the relationships in the Job DemandControl-Support Model play out in a similar sample as the proposed APIMs (i.e. the full employee population), two post-hoc models were examined using hierarchical multiple regression analyses. Three steps were taken to address the three-way interaction proposed in the JDCS Model. None of the three steps were significant when predicting physical health or when predicting mental health. As mentioned previously, there are mixed results on whether or not proposed interactions in the JDCS Model are supported. Even high-quality studies only provide modest support for the interactional effect of demands and control (de Lange et al., 2003). De Jonge and colleagues (2000) were only able to support interactive effects in specific occupational groups. The present study failed to provide evidence of interactive effects between job demands, control and support in a population of grocery employees. These results further contribute to the confusion in the literature.

\section{Contributions of the Present Study}

Primary contributions. The present dissertation offers two primary contributions to the Occupational Health Psychology field. First, the study included hypotheses about crossover relationships that had the potential to contribute to the expanding Crossover literature. Although the connection between job demands and employee health has been well-established, no crossover researchers have addressed the potential crossover effect on the partner's health. The direct crossover relationships between job demands of one partner and health outcomes of the other partner were not supported as hypothesized. The examination of these relationships still proves useful to the state of the crossover literature as it is beneficial to know that these relationships do not exist in the current 
population of predominantly low-wage and hourly workers. Further, when examining post-hoc models, it was found that there was a significant crossover of women's job satisfaction on men's levels of mental health. This is an exciting finding that has not been demonstrated in the crossover literature.

The second primary contribution involves the proposed typology of crossover research. This system for categorizing crossover studies was proposed in order to add structure to future research and discussions in the crossover literature. The goal was to address the lack of uniformity in how literature reviews and crossover results are reported. This typology proved useful for the present study by providing a structure with which to discuss the crossover hypotheses and results. This model could be tested in future research as well. It provides researchers with the ability to hypothesize crossover relationships that fall into each of the categories of the typology. If crossover findings are discovered that do not fit into the typology; revisions could then be made to the typology.

The proposed typology also has the potential to guide future research by identifying what areas of crossover research future researchers should focus on. For example, the large majority of crossover studies address the crossover of job demands, specifically the crossover of stressors on strain outcomes (stressor $\rightarrow$ strain) and the crossover of strain between partners (strain $\rightarrow$ strain). The greatest need for future research lies in the area of crossover of resources. Only 17 out of 72 research studies have discovered crossover effects of resources between partners at home or in the workplace. Further, the present study provides a good starting point for a potential metaanalysis of the crossover literature. The comprehensive review and organization of the 
entire crossover literature via the proposed typology would contribute greatly to a potential meta-analysis.

Secondary contributions. Secondary contributions include examining crossover of health between partners, using an advanced method of dyadic data analysis (APIM) and researching an understudied population of low-wage dual-earner couples.

Crossover of health. A secondary contribution includes examining crossover of health between partners. Some researchers have examined crossover of general health (Westman et al., 2008) while others have examined physical health (GorgievskiDuijvesteijn et al., 2000; Jones \& Fletcher, 1993b) or a facet of mental health like depression or anxiety (Crossfield et al., 2005; Jones \& Fletcher, 1993a). The present study offers the benefit of including both a composite score of physical health and mental health, thus examining whether there is a crossover of the strain of mental health and a crossover of the strain of physical health within the same population. Although many of the proposed crossover relationships were not supported, it was found that there was a significant crossover of physical health between partners, as well as a significant crossover of mental health between partners. This is the first study to find significant crossover of both a physical health and a mental health composite score between partners.

Further, the inclusion of both a physical health and mental health composite score in the analyses helps clarify the potential crossover relationships between the predictor of interest, job demands, and the different aspects of health. One interesting within-person finding was that men's job demands were positively and significantly associated men's mental health. Conversely, men's job demands had a significant negative relationship with physical health. These results suggest that having a challenging job is mentally 
stimulating to men, thus increasing their mental health composite score, yet job demands are still physically draining to the male population in this study.

The present study not only examines direct crossover relationships between partners but also takes into account the possibility of common stressors. Income adequacy may play an important role in the population of interest: a low-wage sample of grocery employees. It was critical to take into account the possibility that income adequacy could be a common stressor for both partners that simultaneously affects their level of strain. If the study models failed to take the role of this common stressor into account, it would not have been possible to rule out the potential third-variable effect of income adequacy. For example, if the hypothesized direct crossover relationships had been significant, it would have been helpful to know that the common stressor of income adequacy was not biasing the results. Income adequacy was not found to be simultaneously predictive of the health outcomes for men and women, thus it did not serve as a common stressor variable.

Although the common stressor mechanism was not supported, it was helpful to include income adequacy in the analyses to better understand the relationships between variables. For instance, it was found that mental health for men was significantly predicted by men's income adequacy. Since none of the other proposed relationships involving income adequacy were significant, this tells us something about our population. Income adequacy of partners was strongly associated, so it is possible that men take on the stress of finances to a higher degree than women in this population. The stress related to income adequacy manifests itself in men's mental health but is not related to female health variables. 
Dyadic data analysis. Another secondary contribution of this study is the use of an advanced method of dyadic data analysis. The models tested in the present study address crossover between spouses using a data analytic method that allows researchers to investigate issues of mutual influence. The Actor-Partner Interdependence Model (APIM) is a model of dyadic relationships that integrates a conceptual view of interdependence in two person relationships (Cook \& Kenny, 2005). Kashy and Kenny (2000) suggest using APIMs to help control for the non-independence of data that naturally occurs in relationship dyads. Although the methodology is considered a benefit of the present study; using APIMs does lead to more conservative estimates than conducting separate regression analyses for each hypothesis.

Low-wage dual-earner population. A final secondary contribution of the present study involves researching an understudied population of low-wage dual-earner couples. As of $2008,61 \%$ of women and $56 \%$ of men holding wage and salaried positions were paid by the hour. Further, a disproportionate amount of those hourly workers are in lowpaying jobs (BLS, 2009a). Despite the strong presence of low-wage and hourly workers in the total workforce, crossover research has failed to directly address this population. The present study is the first crossover research to focus on a population of low-wage, hourly workers in the service industry. Although many studies are sampled randomly from the general population or a subset of the population, thus providing representation of a wide variety of workers, no other crossover researchers have focused on a population of low-wage or hourly workers.

It is important to note that although this is the first study to directly address crossover in low-income or hourly workers, not all crossover studies focus on a 
professional or high-income population. Some of the research studies, whether sampled randomly or not, did have a higher concentration of low-income households included than others (e.g. Cronkite \& Moos, 1984; Kossek et al., 2008; Riley \& Eckenrode, 1986; Westman et al., 2009). One study examined crossover in a sample of dairy farmers (Gorgievski-Duijvesteijn et al., 2000). This sample may qualify as low-wage workers but they are a substantively different group than the representative group of low-wage workers common in the United States and other developed countries. Along the same lines, other crossover research studies have focused on populations that are not professional workers (e.g. police officers, teachers, graduate students, military personnel). Although these are important populations to examine, they did not serve the purpose of being representative of the large percentage of workers in the U.S. and beyond that are low-wage or hourly workers in the service industry. Further, crossover studies that have also addressed the transference of stress related to one member of the dyad being unemployed (e.g. Howe et al., 2004; Westman et al., 2004). Although these studies address a population with financial issues, the stress is not necessarily a factor of working in low-wage or hourly jobs.

The present study is significantly contributing to the crossover literature by exploring crossover relationships in a population focused on hourly, low-wage workers. These workers are representative of the growing population of employees working in the expanding service industry. In 2008, service industry workers comprised $19.6 \%$ of the overall employment population (BLS, 2009). Further, employment in service occupations is projected to increase by 13.8 percent from 2008 to 2018 (BLS, 2009b). More 
specifically, grocery stores ranked among the largest industries in 2008, providing 2.5 million wage-and-salary jobs (BLS, 2010).

There are several important differences that the low-wage or hourly workers can have in comparison to the rest of the working population. First, they are much less likely to have health insurance coverage paid at least in part by their employers than their higher-wage employee counterparts (Families and Work Institute, 2006). This causes a major problem based on the fact that they are unlikely to be able to purchase insurance at market rate. Thus, unless they are covered by public programs or a family member's policy, many remain uninsured. One third (33\%) of low-wage and low-income employees do not have health insurance coverage from any source whatsoever (Stoll, 2005). Second, employers in today's economy face strong pressure to minimize labor costs, especially in the service sector, where labor is a principal expenditure (Lambert, 2008). Workers at the front lines often feel the impact of fluctuations in demand for services through reductions in hours (Lambert \& Henly, 2009). This leads to many hourly jobs being unstable and unpredictable. Third, besides health insurance, low-wage or lowincome workers are less likely to receive numerous other job benefits. They are much less likely to receive paid time off for personal illness, receive paid vacation days or be offered any formal training or education for job skills improvement than mid- and higherwage employees (Families and Work Institute, 2006).

\section{Practical Implications}

Exploring how work stressors affect the health of the employee and his or her partner can help practitioners understand the far-reaching impacts of job design. Features of job design include psychological demands like how hard and fast employees must 
work, having enough time to complete the work or being free from conflicting demands. There are several ways that understanding the full effects of stressful work environments can have applied value in the workplace. It is crucial to ensure that job design is appropriate to encourage general health, not only because of the costs associated with health problems for the employee (e.g. absenteeism, productivity, turnover), but also because of the potential health problems related to the spouse or partner that may affect the company (e.g. increased family-to-work conflict, health insurance costs). Further, these issues are especially relevant in low-wage or hourly populations; in which employees may not have access to resources to deal with additional stressors (Heymann, 2006). In order to see maximum benefit of this applied research, academics and researchers must invest time and effort to ensure management understands the value of improving the work environment for employees.

\section{Potential Limitations}

Although the results of the study extend previous literature, it is appropriate to recognize potential limitations. First of all, a cross-sectional design was employed. This design does not allow researchers to make conclusive inferences concerning the precedence of the relationships depicted in the model. Secondly, the use of all self-report data suggests the possibility of common method bias, meaning that the variance in the measurement of constructs could possibly be attributed to the instrumentation used rather than to the constructs of interest.

A third possible limitation is that the nature of this sample could potentially limit the applicability of the findings to other settings. Although the purpose of this study was to examine low-wage workers, the sample does limit the generalizeability of the results. 
It is not assumed that the findings of the present study would generalize to a population of professional or highly compensated employees. Because of the nature of this occupation, the sample consisted mainly of female employees. This led to a majority of the women in the dyads to be grocery employees, while the men worked in a variety of occupations. This could have potentially led to occupation being a confounding factor. Additionally, many grocery positions are not full-time. This can be evidenced by the lower average number of work hours reported by the females (i.e. 32 hours/week) as compared to the males in the population (i.e. 40 hours/week). The number of part-time workers that were female may be underestimating the effects. For example, job demands may play a different role on strain outcomes for those working full-time versus part-time workers. Further, this effect may be magnified since a greater percentage of the female partners in the dyads were part-time than was the case for the male partners. Another potential limitation is that the hours worked were not controlled for in the APIMs. Considering the high number of part-time grocery employees, this could have an effect on the results. Controlling for hours worked could potentially minimize some of the differences between men and women; since men worked a higher average number of hours per week.

A solution to the potential confounding of gender and occupation would be to set the dataset up to detect crossover from the employee to the partner. Considering the compelling evidence for gender effects in crossover research (Bolger et al., 1989; Burke et al., 1980; Fletcher, 1983; Jackson \& Maslach, 1982; Jackson et al., 1985; Long \& Voges, 1987; Mitchell et al., 1983; Riley \& Eckenrode, 1986), it was decided that accounting for gender effects was more of a priority. It would be beneficial for future 
studies to seek replication in a different industry with a less homogenous sample (e.g. an industry that contained approximately equal distributions of males and females).

The sample does present other possible limitations. The present study has a relatively small sample size, although it does exceed the typical sample size for APIMs. Kenny et al. (2006) stated that the typical sample for an APIM is about 80 dyads. Another possible limitation of the study concerns the complex nature of the models. Kenny, Cashy and Cook (2006) recommend not making models too complex. "A more complex model is not necessarily a better model. Make sure the data are able to answer the questions that they are asked (pp. 425).” Kenny et al. (2006) additionally warned that one additional degree of freedom is lost for every variable that is controlled for. A balance must be found between including relevant variables and decreasing the power of the study.

A final limitation that warrants discussion involves several of the measures used in the study. The scales in the Job Content Questionnaire have borderline acceptable reliability levels. A study is considered acceptable when it includes good references for and acceptable reliabilities of one's variables (alpha of around .70; Stangor, 1998, p. 92). Nunnally (1978) also suggested that the typical cutoff for reliability levels should be .70 . Although the reliabilities for the job demands $(\alpha=.70)$, decision authority $(\alpha=.69)$, and skill discretion ( $\alpha=.71)$ subscales of the JCQ are lower than desired, added benefits of using the JCQ do exist. One advantage of the instrument is that its scales are also used in Karasek's database linkage system (using job title) through which job content scores can be associated with health and productivity outcomes in national or company databases already in existence (such as U.S. Census, Commerce or NCHS data). This helps assure 
the continued validation of the instrument's scales in predicting a broad range of outcome variables.

According to the Job Content Questionnaire Center (2009), most of the scales are nationally standardizable, allowing users involved in small population studies to compare their findings to national averages on the scales (broken down by sex and occupation/industry). The national standardizability is due to the fact that a core of the questions replicate the U.S. Department of Labor's National Quality of Employment Survey of 1969, 1972, and 1977. Many researchers have used the Job Content Questionnaire (JCQ) as a measure of job demands and control and have shown it to be psychometrically acceptable and possess criterion-related validity (e.g. Karasek et al., 1998). I feel confident that the use of the JCQ is justified, with the main drawback being that findings may be attenuated and therefore represent a conservative estimate of the effects of the true relationship.

\section{Suggestions for Future Research}

Future research should extend the examination of crossover beyond the partner. It would be beneficial to understand how stressors and strain of the individual employee affect other family members (i.e. children, parents, grandchildren). According to Systems theory, components within a system interrelate and affect each other (Bronfenbrenner, 1977). Thus, an important next step would be to consider all components in a family system when examining crossover. Studies utilizing a longitudinal research strategy would additionally be an appropriate avenue for future research. Longitudinal research would help researchers better understand crossover effects and causality more rigorously. 
This would be especially relevant when addressing health outcomes, as some effects on health and well-being may become more apparent over time.

Future research could also examine crossover of a variety of other job stressors on health outcomes. There are several variables that have demonstrated a connection to health outcomes within-person that would be interesting to include in a crossover model. Job control, another important predictor discussed in the Job Demand-Control Model, has been demonstrated to have a strong connection with health outcomes. Loss of decision latitude, including authority over decisions and skill discretion, is related to numerous physical health outcomes such as myocardial infarctions, low back pain, and acute neck and shoulder pain (Theorell, 2003). Decision latitude influences health risk through lifestyle factors (e.g. smoking, diet, substance use), and direct effects on endocrine system and metabolism (Theorell, 2003). Addressing the psychological component of overall health, Holman and Wall (2002) found that job control has a direct effect on depression levels of employees. Similarly, research by Wall, Jackson, Mularkey, and Parker (1996) found that three measures of control at work, timing control, method control and decision latitude, significantly predicted levels of depression and anxiety.

Various operationalizations of financial stress have been connected to physiological and psychological health of employees (Hu et al., 2007; Mills et al., 1992; Olivius et al., 2004; Pierce et al., 1994), as well as SES (Franks et al., 2003) and dissatisfaction with one's financial situation (Liang et al., 1999). It would be interesting to see how these variables cross over between partners. Organizational justice also has been shown to have an impact on health of employees (Elovainio et al. 2002; Kivimaki et al., 2003; Taris et al., 2002; Tepper, 2001). Future research could examine how the stress 
of low organizational justice affects the partner's health outcomes. Another interesting predictor variable to consider in future research is emotional labor. Researchers have connected the surface acting component of emotional labor with negative health-related outcomes like burnout and depression (Grandey, 2003).

Future research could examine the proposed models while taking into account other related variables. For example, it would be beneficial to control for the effects of work values, attitude or personality characteristics like negative affectivity. The relationship between job characteristics and health may be impacted by how much emphasis the individual places on work or how they perceive the role of work in their life. As well as being a possible control variable, negative affect (NA) could potentially play a mediating role, as research has demonstrated a connection between work stressors and NA, as well as a connection between NA and health complaints (Watson, 1988).

Other mediators that would be valuable to examine include potential explanatory mechanisms for the crossover process. Mediators that could be argued to account for the crossover of stressors and strain between partners include empathy, social undermining, communication patterns, marital quality or years together, personality, anger or other moods and emotions. Researchers have examined some of these potential explanatory mechanisms (e.g. Bakker et al., 2008; Bakker \& Schaufeli, 2000; Bakker et al., 2007; Lavee \& Ben-Ari, 2007) but no research has included these variables in the models hypothesized in the present study.

Further, it would be interesting to examine potential moderators in the hypothesized models. It would be valuable to examine how social support or a related construct (e.g. peer or coworker support) differed for male or female partners and 
whether it plays a buffering role in the relationship between job characteristics and health outcomes. This potential moderator would be especially relevant for the present study's focus on low-wage, hourly grocery employees. Another interesting avenue would be to explore the potential moderating or mediating role of the individuals' moods. It is possible that moods account for the crossover process between job stressors and health outcomes, or positive moods could serve as a buffer between the negative effects of job stressors and the strain outcomes.

A productive avenue for future research would be to examine the proposed models in a sample where gender and occupation are not so closely linked. A majority of the female partners in the present sample were grocery store employees (e.g. $73.3 \%$ ) while the male partners worked in a variety of occupations. This could have potentially led to occupation being a confounding factor. Another possibility would be to examine the proposed crossover relationships in a sample of salaried employees. It would be beneficial to examine whether there is a stronger connection between job characteristics (e.g. job demands) and health outcomes for those in more career-focused occupations. Further, since the population examined was primarily low-wage and hourly, it would be interested to examine the hypotheses in a sample of more highly compensated employees and spouses.

Finally, as mentioned previously, future research should employ the proposed typology of crossover research to determine where the gaps in the crossover literature are. A productive avenue for future research would be to focus on examining the crossover of resources. Only 17 out of 72 research studies have discovered crossover effects of resources between partners at home or in the workplace. More specifically, only two 
studies have addressed the crossover of resources for one individual on stressors of the partner (resource $\rightarrow$ stressor) and only four studies have supported a crossover effect of one individual's resources on the partner's strain (resource $\rightarrow$ strain).

Summary. In conclusion, this study offered two primary contributions to the literature. First, new direct crossover relationships were examined (i.e. the crossover of one individual's job demands on the partner's health outcomes). There is a demonstrated connection between job demands and health within-person; yet the present study was the first to examine the potential crossover role of employee job demands on spousal health. Although these direct crossover hypotheses were not supported, valuable information is still provided about the population of interest (i.e. primarily low-wage and hourly workforce of dual-earner couples). Further, post-hoc analyses demonstrated a significant crossover of job satisfaction of the female partners on men's mental health composite scores; a new contribution to the crossover literature. Further, in order to examine the crossover hypotheses, within-person relationships were included in the hypothesized Actor-Partner Interdependence Models. Results demonstrated that some well-established relationships were not supported in the present sample. For example, it was found that job demands and health outcomes were not associated for the female partners. Since the women in the study were predominantly grocery store employees, these findings provide useful information about the hourly workforce. A new typology of crossover research was proposed to address the lack of uniformity in how literature reviews and crossover results are reported; an additional primary contribution of this study. The goal was to provide a comprehensive review of crossover literature and propose a comprehensive typology for future discussion and organization of crossover literature. 
Several secondary contributions to the literature were also offered. First, crossover relationships of composite scores of health between partners were demonstrated; both physical health and mental health. Second, an advanced method of dyadic data analysis was used that does not try to get around violations of the assumption of independence. This method was used despite the fact that it could have led to more conservative estimates; which suggests that the significant crossover results are even more valuable. APIMs embrace the interdependence that is an unavoidable part of studying the impact of job characteristics on the family system. Third, the nature of the sample adds to the literature by examining the understudied population of low-wage dualearner couples. The present study is the first crossover research to focus on a low-wage and hourly population of workers. It is valuable to understand how crossover relationships manifest themselves in a population representative of the many low-wage or hourly workers currently in the service industry and other parts of the American economy. 
Table 1

Means and Standard Deviations for APIM Demographics

\begin{tabular}{lrrr}
\hline & $N$ & Mean & SD \\
\hline Male Participants & & & \\
Age & 90 & 43.32 & 12.37 \\
Hours worked per week & 80 & 40.08 & 10.10 \\
Number of children living at home & 86 & 1.30 & 1.28 \\
FSSB Overall Scale & 87 & 3.06 & .81 \\
Job Demands & 81 & 2.36 & .49 \\
Physical Health Composite Score & 90 & 49.98 & 10.29 \\
Mental Health Composite Score & 90 & 48.60 & 11.41 \\
Female Participants & & & \\
Age & 90 & 39.50 & 12.76 \\
Hours worked per week & 87 & 32.25 & 10.14 \\
Number of children living at home & 87 & 1.31 & 1.33 \\
FSSB Overall Scale & 90 & 3.21 & .85 \\
Job Demands & 87 & 2.34 & .44 \\
Physical Health Composite Score & 89 & 50.15 & 10.36 \\
Mental Health Composite Score & 89 & 49.45 & 8.58 \\
\hline
\end{tabular}


Table 2

Frequencies and Percentages: APIM Demographics for Males

\begin{tabular}{|c|c|c|c|}
\hline & $N$ & Frequency & Percentage \\
\hline Race & 90 & & \\
\hline White & & 82 & 91.1 \\
\hline Black or African-American & & 2 & 2.2 \\
\hline American Indian or Alaskan Native & & 4 & 4.4 \\
\hline Other & & 2 & 2.2 \\
\hline Hispanic or Latino & 88 & & \\
\hline No & & 85 & 94.4 \\
\hline Yes & & 3 & 3.3 \\
\hline Education & 90 & & \\
\hline High School Diploma or GED & & 41 & 45.6 \\
\hline Some College or Associate's Degree & & 30 & 33.3 \\
\hline Bachelor's Degree & & 11 & 12.2 \\
\hline Graduate Degree & & 2 & 2.2 \\
\hline Household Income & 89 & & \\
\hline Less than $\$ 25,000$ & & 19 & 21.1 \\
\hline$\$ 25,000-\$ 40,000$ & & 31 & 34.4 \\
\hline$\$ 40,000-\$ 55,000$ & & 22 & 24.4 \\
\hline$\$ 55,000-\$ 70,000$ & & 10 & 11.1 \\
\hline$\$ 70,000-\$ 85,000$ & & 2 & 2.2 \\
\hline Over $\$ 85,000$ & & 5 & 5.6 \\
\hline Income Adequacy & 89 & & \\
\hline We can't make ends meet & & 9 & 10.1 \\
\hline We have just enough, no more & & 28 & 31.5 \\
\hline We have enough, with a little extra, sometimes & & 45 & 50.6 \\
\hline We always have money left over & & 7 & 7.9 \\
\hline
\end{tabular}


Table 3

Frequencies and Percentages: APIM Demographics for Females

\begin{tabular}{|c|c|c|c|}
\hline & $N$ & Frequency & Percentage \\
\hline Race & 90 & & \\
\hline White & & 87 & 96.7 \\
\hline Black or African-American & & 1 & 1.1 \\
\hline American Indian or Alaskan Native & & 1 & 1.1 \\
\hline Other & & 1 & 1.1 \\
\hline Hispanic or Latino/Latina & 88 & & \\
\hline No & & 87 & 96.7 \\
\hline Yes & & 1 & 1.1 \\
\hline Education & 90 & & \\
\hline Some High School & & 5 & 5.6 \\
\hline High School Diploma or GED & & 45 & 50 \\
\hline Some College or Associate's Degree & & 31 & 34.4 \\
\hline Bachelor's Degree & & 8 & 8.9 \\
\hline Graduate Degree & & 1 & 1.1 \\
\hline Household Income & 89 & & \\
\hline Less than $\$ 25,000$ & & 24 & 26.7 \\
\hline$\$ 25,000-\$ 40,000$ & & 32 & 35.6 \\
\hline$\$ 40,000-\$ 55,000$ & & 15 & 16.7 \\
\hline$\$ 55,000-\$ 70,000$ & & 13 & 14.4 \\
\hline$\$ 70,000-\$ 85,000$ & & 1 & 1.1 \\
\hline Over $\$ 85,000$ & & 2 & 2.2 \\
\hline Income Adequacy & 89 & & \\
\hline We can't make ends meet & & 7 & 7.8 \\
\hline We have just enough, no more & & 36 & 40 \\
\hline We have enough, with a little extra, sometimes & & 40 & 44.4 \\
\hline We always have money left over & & 7 & 7.8 \\
\hline
\end{tabular}


Table 4

Intercorrelations between Study Variables

\begin{tabular}{|c|c|c|c|c|c|c|c|c|c|c|c|c|c|c|}
\hline Variables & 1 & 2 & 3 & 4 & 5 & 6 & 7 & 8 & 9 & 10 & 11 & 12 & Mean & SD \\
\hline 1. Job Demands (M) & -- & & & & & & & & & & & & 2.36 & .49 \\
\hline 2. Job Demands (F) & $.27^{*}$ & -- & & & & & & & & & & & 2.34 & .44 \\
\hline 3. Income Adeq (M) & .07 & -.09 & -- & & & & & & & & & & 2.56 & .78 \\
\hline 4. Income Adeq $(F)$ & .07 & -.11 & $.53 * *$ & -- & & & & & & & & & 2.52 & .75 \\
\hline 5. Physical Health (M) & -.22 & .09 & .19 & .17 & -- & & & & & & & & 49.99 & 10.29 \\
\hline 6. Physical Health (F) & .09 & .07 & -.04 & -.01 & $.28 * *$ & -- & & & & & & & 50.61 & 9.46 \\
\hline 7. Mental Health (M) & $.23^{*}$ & -.10 & $.36^{* *}$ & $.29 * *$ & -.01 & .11 & -- & & & & & & 48.60 & 11.41 \\
\hline 8. Mental Health $(\mathrm{F})$ & -.04 & -.13 & .08 & .15 & -.01 & $-.21^{*}$ & $.30 * *$ & -- & & & & & 49.45 & 8.58 \\
\hline 9. Job Satisfaction (M) & $.29 * *$ & -.08 & $.27 *$ & $.22 *$ & .07 & .16 & $.32 *$ & .11 & -- & & & & 3.19 & 1.44 \\
\hline 10. Job Satisfaction (F) & .13 & -.03 & -.16 & -.14 & .00 & .18 & .17 & .14 & .05 & -- & & & 3.29 & 1.06 \\
\hline 11. Sup Support (M) & .24 & .09 & .21 & .07 & $.22 *$ & .09 & $.24 *$ & .13 & .17 & .10 & -- & & 3.06 & .81 \\
\hline 12. Sup Support (F) & .17 & -.06 & .10 & -.04 & .11 & .08 & .17 & .16 & -.03 & $.34 * *$ & $.43 * *$ & -- & 3.21 & .85 \\
\hline
\end{tabular}

Note. ${ }^{*} p<.05,{ }^{*} p<.01$. Bolded items indicate interdependence scores. $\mathrm{M}=$ scale for male participants, $\mathrm{F}=$ scale for female participants, Income Adeq = Income Adequacy, Sup Support = Supervisor Support. Job Demands were measured using a 4point Likert scale, $1=$ strongly disagree and $4=$ strongly agree. Income Adequacy was measured using a single item indicator of ability to get along on income. Physical and Mental Health were measured using composite scores derived from the SF-12, with responses ranging from 1 (none of the time) to 5 (all of the time). Job Satisfaction and Supervisor Support were measured using a 5-point Likert scale, $1=$ strongly disagree and $5=$ strongly agree. 
Table 5

Unstandardized Effects, Standardized Effects, Correlations, and Significance Levels for APIM in Figure $1(N=90)$

\begin{tabular}{|c|c|c|c|}
\hline Parameter Estimate & Unstandardized & Standardized & $P$ \\
\hline \multicolumn{4}{|l|}{ Within-Person Estimates } \\
\hline Job Demands (M) $\rightarrow$ Physical Health (M) & -5.2 & -.25 & .02 \\
\hline Income Adequacy (M) $\rightarrow$ Physical Health (M) & 2.81 & .21 & .03 \\
\hline Job Demands $(\mathrm{F}) \rightarrow$ Physical Health $(\mathrm{F})$ & 1.65 & .07 & .53 \\
\hline Income Adequacy $(\mathrm{F}) \rightarrow$ Physical Health $(\mathrm{F})$ & -.39 & -.03 & .79 \\
\hline \multicolumn{4}{|l|}{ Crossover Estimates } \\
\hline Job Demands (M) $\rightarrow$ Physical Health (F) & 3.80 & .16 & .13 \\
\hline Job Demands $(\mathrm{F}) \rightarrow$ Physical Health $(\mathrm{M})$ & 1.35 & .06 & .58 \\
\hline Correlations & & $R^{2}$ & $P$ \\
\hline \multicolumn{4}{|l|}{ Within-Person Correlations } \\
\hline Job Demands $(\mathrm{M}) \rightarrow$ Income Adequacy $(\mathrm{M})$ & & .05 & .65 \\
\hline Job Demands (F) $\rightarrow$ Income Adequacy (F) & & -.11 & .32 \\
\hline \multicolumn{4}{|l|}{ Crossover Correlations } \\
\hline Job Demands (M), Job Demands (F) & & .25 & .03 \\
\hline Income Adequacy (M), Income Adequacy (F) & & .53 & .00 \\
\hline Physical Health (M), Physical Health (F) & & .31 & .01 \\
\hline Job Demands (M), Income Adequacy (F) & & .06 & .56 \\
\hline Job Demands (F), Income Adequacy (M) & & -.10 & .35 \\
\hline
\end{tabular}

Note: $\chi^{2}=1.22, \mathrm{p}=.54, \mathrm{CFI}=1.0, \mathrm{RMSEA}=.00$ 
Table 6

Unstandardized Effects, Standardized Effects, Correlations, and Significance Levels for APIM in Figure $2(N=90)$

\begin{tabular}{lrrr}
\hline Parameter Estimate & Unstandardized & Standardized & $P$ \\
\hline Within-Person Estimates & & & \\
Job Demands (M) $\rightarrow$ Mental Health (M) & 4.98 & .22 & .04 \\
Income Adequacy (M) $\rightarrow$ Mental Health (M) & 4.92 & .34 & .00 \\
Job Demands (F) $\rightarrow$ Mental Health (F) & -2.48 & -.13 & .26 \\
Income Adequacy (F) $\rightarrow$ Mental Health (F) & 1.31 & .12 & .26 \\
Crossover Estimates & & & \\
Job Demands (M) $\rightarrow$ Mental Health (F) & -.36 & -.02 & .86 \\
Job Demands (F) $\rightarrow$ Mental Health (M) & -2.60 & -.10 & .33 \\
\hline Correlations & & $R^{2}$ & $P$ \\
\hline Within-Person Correlations & & & \\
Job Demands (M) $\rightarrow$ Income Adequacy (M) & & .10 & .39 \\
Job Demands (F) $\rightarrow$ Income Adequacy (F) & & -.10 & .34 \\
Crossover Correlations & & & \\
Job Demands (M), Job Demands (F) & & .28 & .02 \\
Income Adequacy (M), Income Adequacy (F) & .53 & .00 \\
Mental Health (M), Mental Health (F) & .28 & .01 \\
Job Demands (M), Income Adequacy (F) & .09 & .41 \\
Job Demands (F), Income Adequacy (M) & -.10 & .36 \\
\hline
\end{tabular}

Note: $\chi^{2}=1.08, \mathrm{p}=.58, \mathrm{CFI}=1.00, \mathrm{RMSEA}=.00$ 
Table 7

Demographics for Post-Hoc Analyses addressing JDCS Model

\begin{tabular}{|c|c|c|c|}
\hline & $N$ & Mean & SD \\
\hline \multicolumn{4}{|l|}{ Means and Standard Deviations } \\
\hline Age & 359 & 38.23 & 15.25 \\
\hline Hours worked per week & 360 & 31.36 & 8.55 \\
\hline Number of children living at home & 341 & .8 & 1.09 \\
\hline Work-to-Family Conflict & 358 & 2.62 & .88 \\
\hline Family-to-Work Conflict & 358 & 1.92 & .56 \\
\hline FSSB Overall Scale & 360 & 3.44 & .71 \\
\hline \multirow[t]{2}{*}{ Parent care hours per week } & 54 & 19.69 & 24.83 \\
\hline & $N$ & Frequency & Percentag \\
\hline \multicolumn{4}{|l|}{ Frequencies and Percentages } \\
\hline Gender & 359 & & \\
\hline Male & & 97 & 26.9 \\
\hline Female & & 262 & 72.8 \\
\hline Race & 358 & & \\
\hline White & & 330 & 91.7 \\
\hline Black or African-American & & 14 & 3.9 \\
\hline American Indian or Alaskan & & 2 & .6 \\
\hline Asian & & 1 & .3 \\
\hline Native Hawaiian or Pacific & & 2 & 6 \\
\hline Other & & 9 & 2.5 \\
\hline Hispanic or Latino & 329 & & \\
\hline No & & 316 & 87.8 \\
\hline Yes & & 13 & 3.6 \\
\hline Education & 358 & & \\
\hline Some High School & & 10 & 2.8 \\
\hline High School Diploma or GED & & 196 & 54.4 \\
\hline Some College or Associate's & & 119 & 33.1 \\
\hline Bachelor's Degree & & 28 & 7.8 \\
\hline Graduate Degree & & 5 & 1.4 \\
\hline Household Income & 346 & & \\
\hline Less than $\$ 25,000$ & & 132 & 36.7 \\
\hline$\$ 25,000-\$ 40,000$ & & 102 & 28.3 \\
\hline$\$ 40,000-\$ 55,000$ & & 50 & 13.9 \\
\hline$\$ 55,000-\$ 70,000$ & & 31 & 8.6 \\
\hline$\$ 70,000-\$ 85,000$ & & 10 & 2.8 \\
\hline Over $\$ 85,000$ & & 21 & 5.8 \\
\hline
\end{tabular}


Table 8

Post-Hoc Hierarchical Multiple Regression Analyses addressing JDCS Model, Part 1

\begin{tabular}{|c|c|c|c|}
\hline Variable & $\Delta R^{2}$ & $F$ Change & $B$ \\
\hline Step 1: & .02 & .09 & \\
\hline Job Demands & & & .01 \\
\hline Decision Authority & & & $.13 *$ \\
\hline Supervisor Support & & & .02 \\
\hline
\end{tabular}

Step 2:

Job Demands * Decision Authority

.00

.88

Job Demands * Supervisor Support

Decision Authority * Supervisor Support
.01

.04

$-.04$

$.00 \quad .99$

Step 3:

Job Demands*Decision Authority*Supervisor

Support

$-.00$

Note. $N=360 .{ }^{*} p<<.01,{ }^{*} p<.05$. All unstandardized regression coefficients are from the third step of the regression analysis. DV $=$ Physical Health composite score. 
Table 9

Post-Hoc Hierarchical Multiple Regression Analyses addressing JDCS Model, Part 2

\begin{tabular}{|c|c|c|c|}
\hline Variable & $\Delta R^{2}$ & $F$ Change & $B$ \\
\hline Step 1: & .01 & 1.31 & \\
\hline Job Demands & & & $.11^{*}$ \\
\hline Decision Authority & & & .00 \\
\hline Supervisor Support & & & .01 \\
\hline Step 2: & .02 & 2.35 & \\
\hline Job Demands * Decision Authority & & & -.08 \\
\hline Job Demands * Supervisor Support & & & .09 \\
\hline Decision Authority * Supervisor Support & & & .08 \\
\hline $\begin{array}{l}\text { Step 3: } \\
\text { Job Demands*Decision Authority*Supervisor } \\
\text { Support }\end{array}$ & .00 & .03 & .01 \\
\hline
\end{tabular}

Note. $N=360 .{ }^{*} * p<.01,{ }^{*} p<.05$. All unstandardized regression coefficients are from the third step of the regression analysis. DV $=$ Mental Health composite score. 
Table 10

Summary of Hypotheses Tested, Part 1

\begin{tabular}{|c|c|c|}
\hline Hypotheses & Support & Conclusion \\
\hline $\begin{array}{l}\text { H1: Demands } \rightarrow \text { Physical } \\
\text { Health }\end{array}$ & Partially & $\begin{array}{l}\text { Psychological demands predict } \\
\text { physical health within person for } \\
\text { male partners but not for female } \\
\text { partners }\end{array}$ \\
\hline $\begin{array}{l}\text { H2: Income Adequacy } \rightarrow \\
\text { Physical Health }\end{array}$ & No & $\begin{array}{l}\text { Income adequacy is not predictive } \\
\text { of physical health within person }\end{array}$ \\
\hline $\begin{array}{l}\text { H3: Demands }(M) \rightarrow \text { Physical } \\
\text { Health }(F) \text {, vice versa }\end{array}$ & No & $\begin{array}{l}\text { Psychological demands of one } \\
\text { partner do not cross over to predict } \\
\text { physical health of the other partner }\end{array}$ \\
\hline $\begin{array}{l}\text { H4: Demands }(\mathrm{M}) \leftarrow \rightarrow \\
\text { Demands }(\mathrm{F})\end{array}$ & Yes & $\begin{array}{l}\text { Psychological demands are } \\
\text { correlated between partners }\end{array}$ \\
\hline $\begin{array}{l}\text { H5: Income Adequacy }(\mathrm{M}) \\
\leftarrow \rightarrow \text { Income Adeq }(\mathrm{F})\end{array}$ & Yes & $\begin{array}{l}\text { Income adequacy is correlated } \\
\text { between partners }\end{array}$ \\
\hline H6: Demands $\leftarrow \rightarrow$ Income & No & $\begin{array}{l}\text { Psychological demands are not } \\
\text { correlated with income adequacy } \\
\text { for male or female partners }\end{array}$ \\
\hline $\begin{array}{l}\text { H7: Demands }(M) \rightarrow \text { Income } \\
\text { Adeq }(F), \text { vice versa }\end{array}$ & No & $\begin{array}{l}\text { Psychological demands of one } \\
\text { partner do not cross over to predict } \\
\text { income adequacy of the other } \\
\text { partner }\end{array}$ \\
\hline $\begin{array}{l}\text { H8: Physical Health }(\mathrm{M}) \leftarrow \rightarrow \\
\text { Physical Health }(\mathrm{F})\end{array}$ & Yes & $\begin{array}{l}\text { Physical health composite scores } \\
\text { are correlated between partners }\end{array}$ \\
\hline
\end{tabular}

Note . Demands = Psychological Demands; $\mathrm{M}=$ scale measurement for Males; $\mathrm{F}=$ scale measurement for Females. 
Table 11

Summary of Hypotheses Tested, Part 2

\begin{tabular}{|c|c|c|}
\hline Hypotheses & Support & Conclusion \\
\hline $\begin{array}{l}\text { H9: Demands } \rightarrow \text { Mental } \\
\text { Health }\end{array}$ & Partially & $\begin{array}{l}\text { Psychological demands predict } \\
\text { mental health within person for } \\
\text { male partners but not for female } \\
\text { partners }\end{array}$ \\
\hline $\begin{array}{l}\text { H10: Income Adequacy } \rightarrow \\
\text { Mental Health }\end{array}$ & Partially & $\begin{array}{l}\text { Income adequacy is predictive of } \\
\text { mental health within person for } \\
\text { male partners but not for female } \\
\text { partners }\end{array}$ \\
\hline $\begin{array}{l}\text { H11: Demands }(\mathrm{M}) \rightarrow \\
\text { Mental Health }(\mathrm{F}), \text { vice } \\
\text { versa }\end{array}$ & No & $\begin{array}{l}\text { Psychological demands of one } \\
\text { partner do not cross over to predict } \\
\text { mental health of the other partner }\end{array}$ \\
\hline $\begin{array}{l}\text { H12: Demands }(\mathrm{M}) \leftarrow \rightarrow \\
\text { Demands }(\mathrm{F})\end{array}$ & Yes & $\begin{array}{l}\text { Psychological demands are } \\
\text { correlated between partners }\end{array}$ \\
\hline $\begin{array}{l}\frac{\mathrm{H} 13}{(\mathrm{M})} \leftarrow \rightarrow \text { Income Adequacy } \\
\text { Adequacy }(\mathrm{F})\end{array}$ & Yes & $\begin{array}{l}\text { Income adequacy is correlated } \\
\text { between partners }\end{array}$ \\
\hline $\begin{array}{l}\text { H14: Demands } \leftarrow \rightarrow \\
\text { Income Adequacy }\end{array}$ & No & $\begin{array}{l}\text { Psychological demands are not } \\
\text { correlated with income adequacy } \\
\text { for male or female partners }\end{array}$ \\
\hline $\begin{array}{l}\text { H15: Demands }(M) \rightarrow \\
\text { Income Adequacy }(F) \text {, vice } \\
\text { versa }\end{array}$ & No & $\begin{array}{l}\text { Psychological demands of one } \\
\text { partner do not cross over to predict } \\
\text { income adequacy of the other } \\
\text { partner }\end{array}$ \\
\hline $\begin{array}{l}\text { H16: Mental Health (M) } \\
\leftarrow \rightarrow \text { Mental Health }(\mathrm{F})\end{array}$ & Yes & $\begin{array}{l}\text { Physical health composite scores } \\
\text { are correlated between partners }\end{array}$ \\
\hline
\end{tabular}

Note. Main effects: Demands = Psychological Demands; DA = Decision Authority; Support $=$ Supervisor Support; $\mathrm{X}$ denotes interaction effect. 
Figure 1. APIM predicting Physical Health.

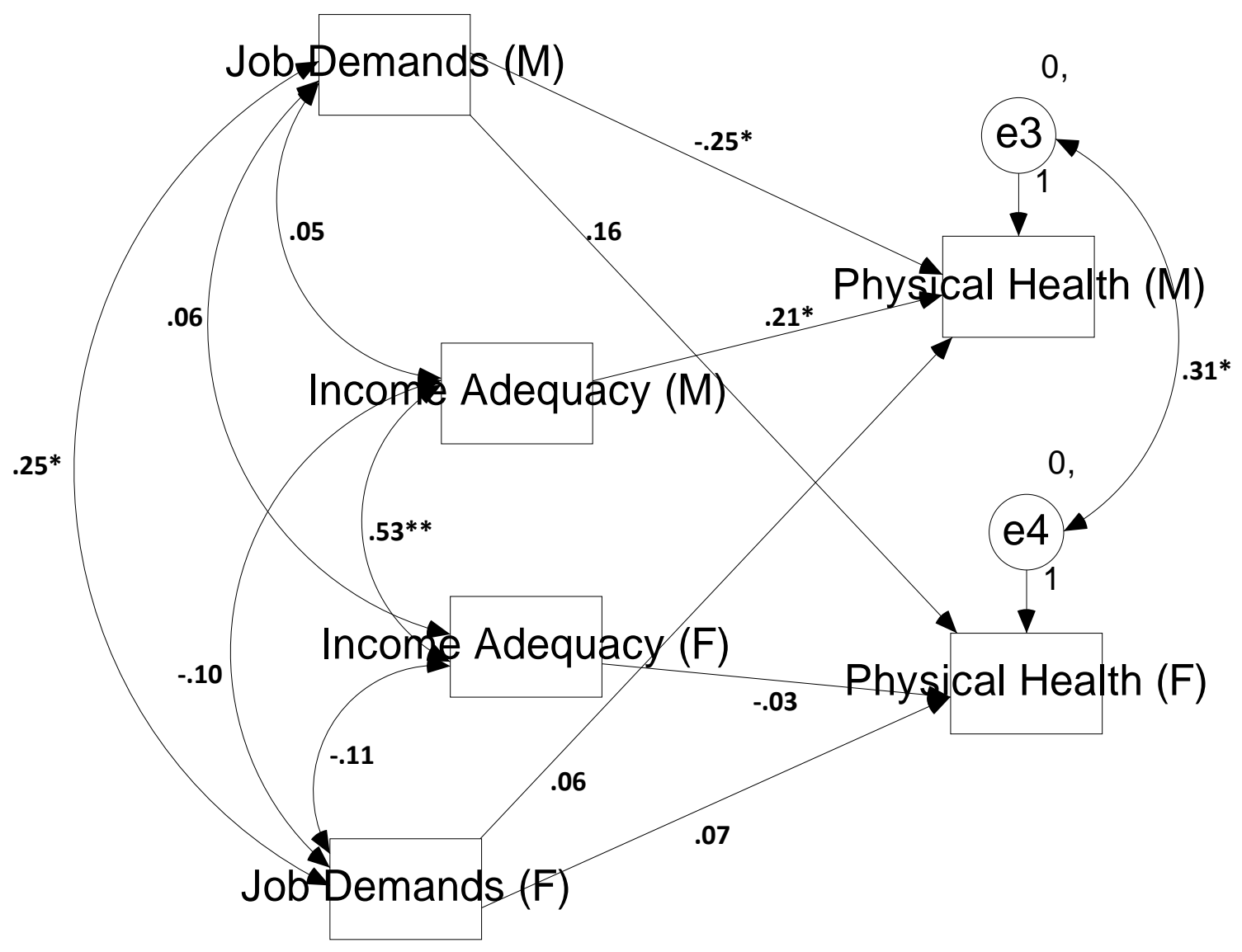

Note: ${ }^{* *} p<.01,{ }^{*} p<.05 . \mathrm{M}=$ scale measurement for Males; $\mathrm{F}=$ scale measurement for Females. 
Figure 2. APIM predicting Mental Health.

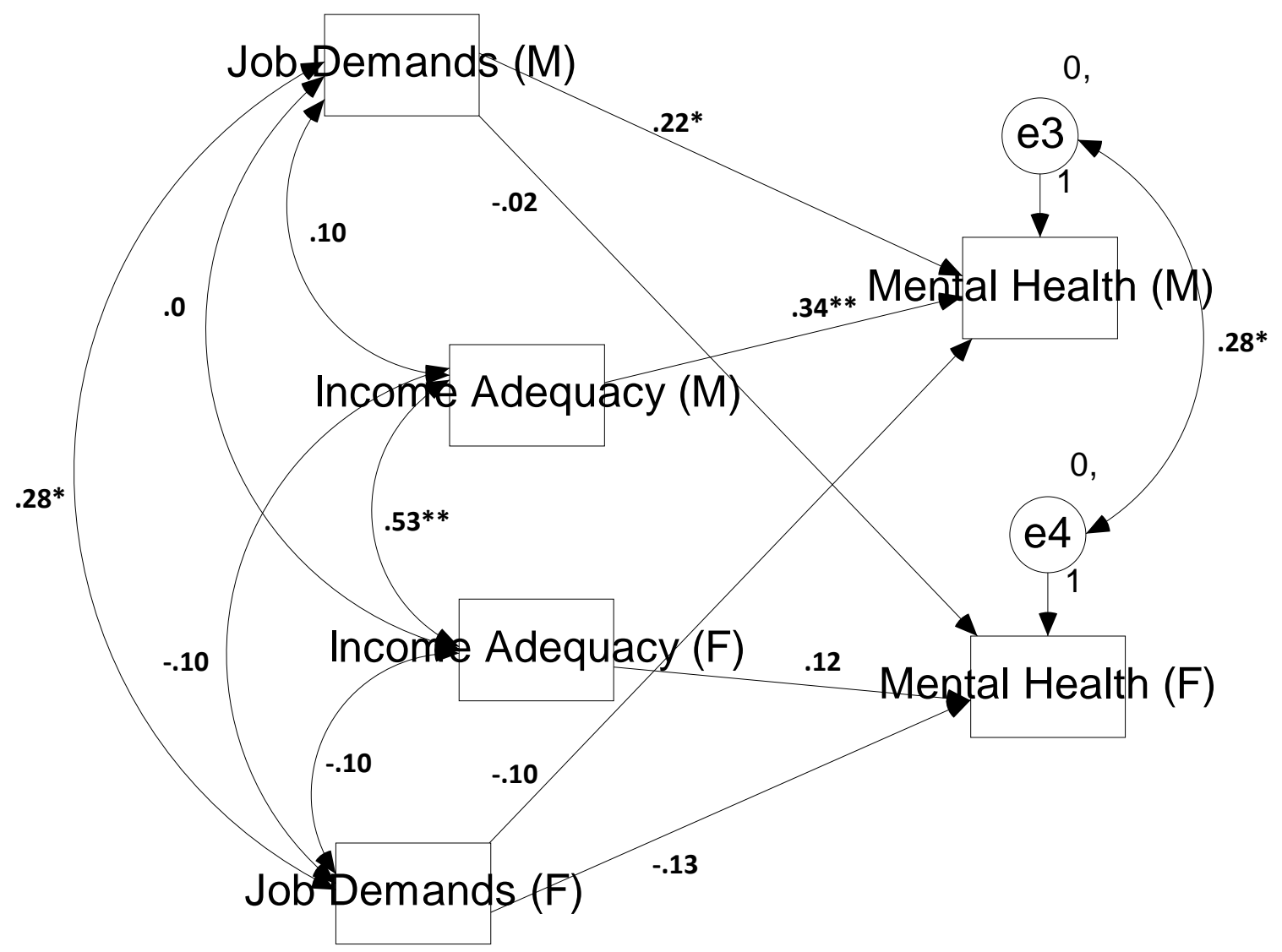

Note: ${ }^{*} p<.01, * p<.05 . \mathrm{M}=$ scale measurement for Males; $\mathrm{F}=$ scale measurement for Females. 
Figure 3. Alternate APIM 1.

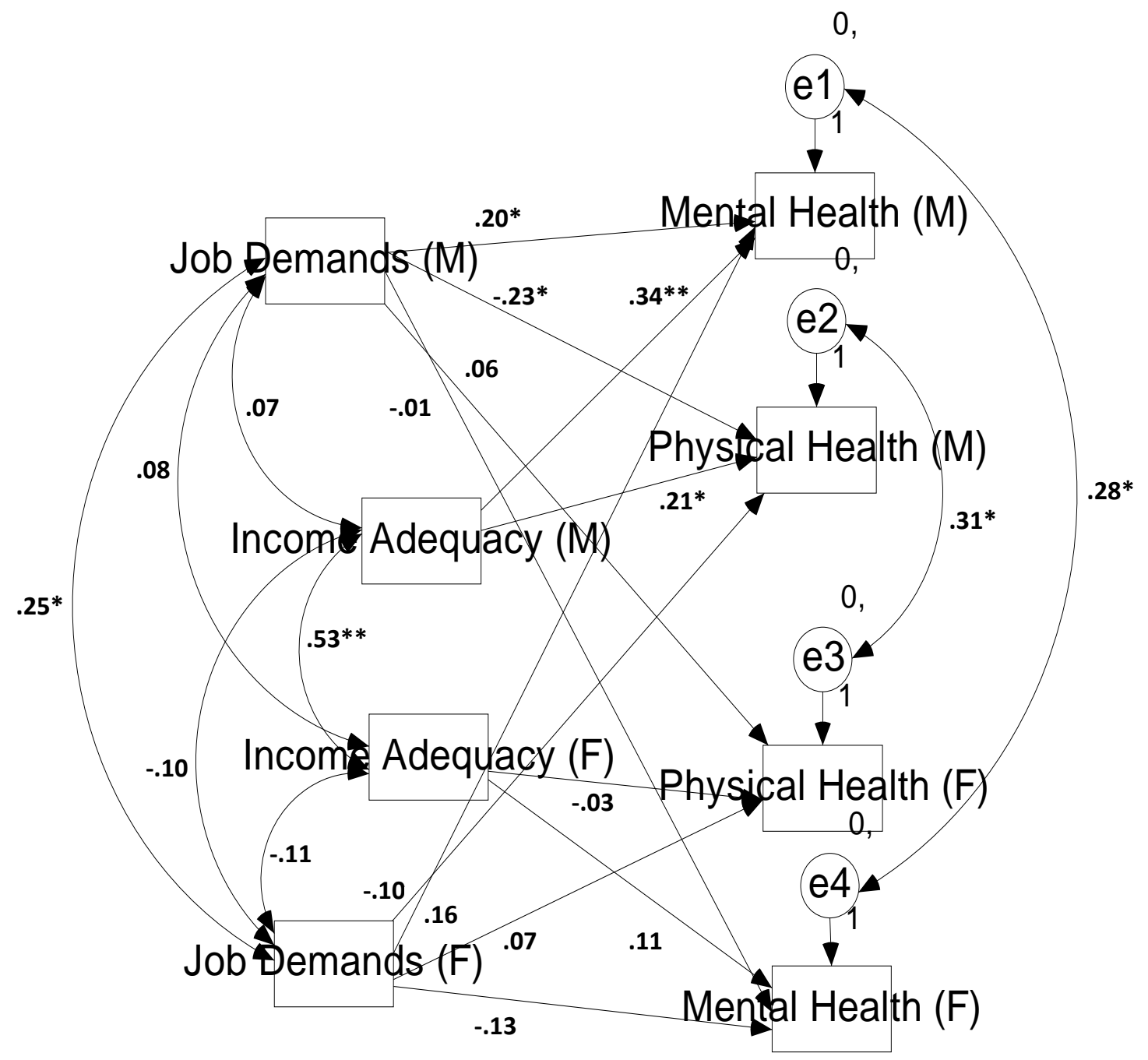

Note: ${ }^{*} p<.01,{ }^{*} p<.05$. Job Sat $=$ Job Satisfaction scale. $\mathrm{M}=$ scale measurement for Males; F = scale measurement for Females. 
Figure 4. Alternate APIM 2.

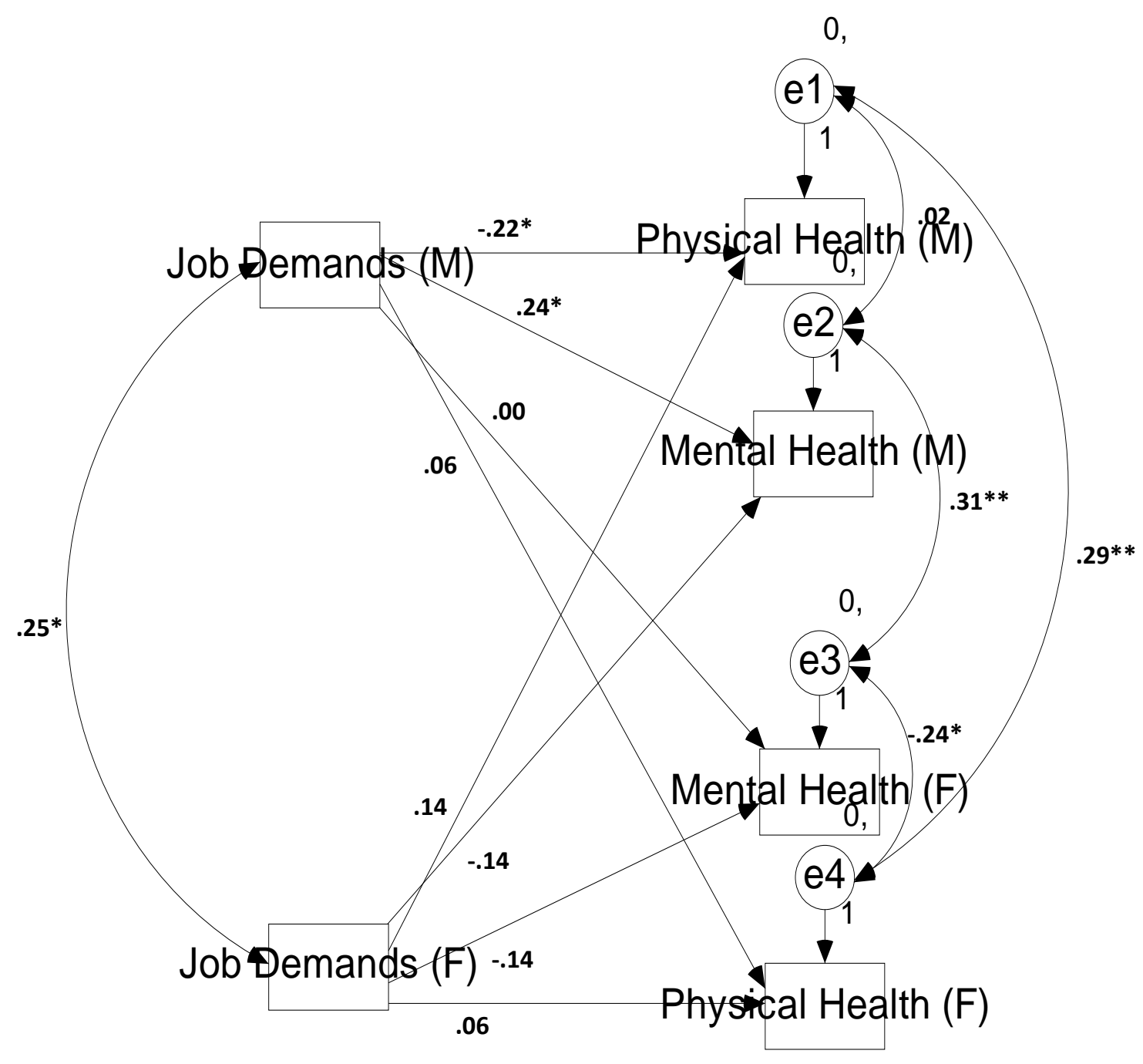

Note: $* * p<.01, * p<.05 . \mathrm{M}=$ scale measurement for Males; $\mathrm{F}=$ scale measurement for Females. 
Figure 5. Alternate APIM 3.

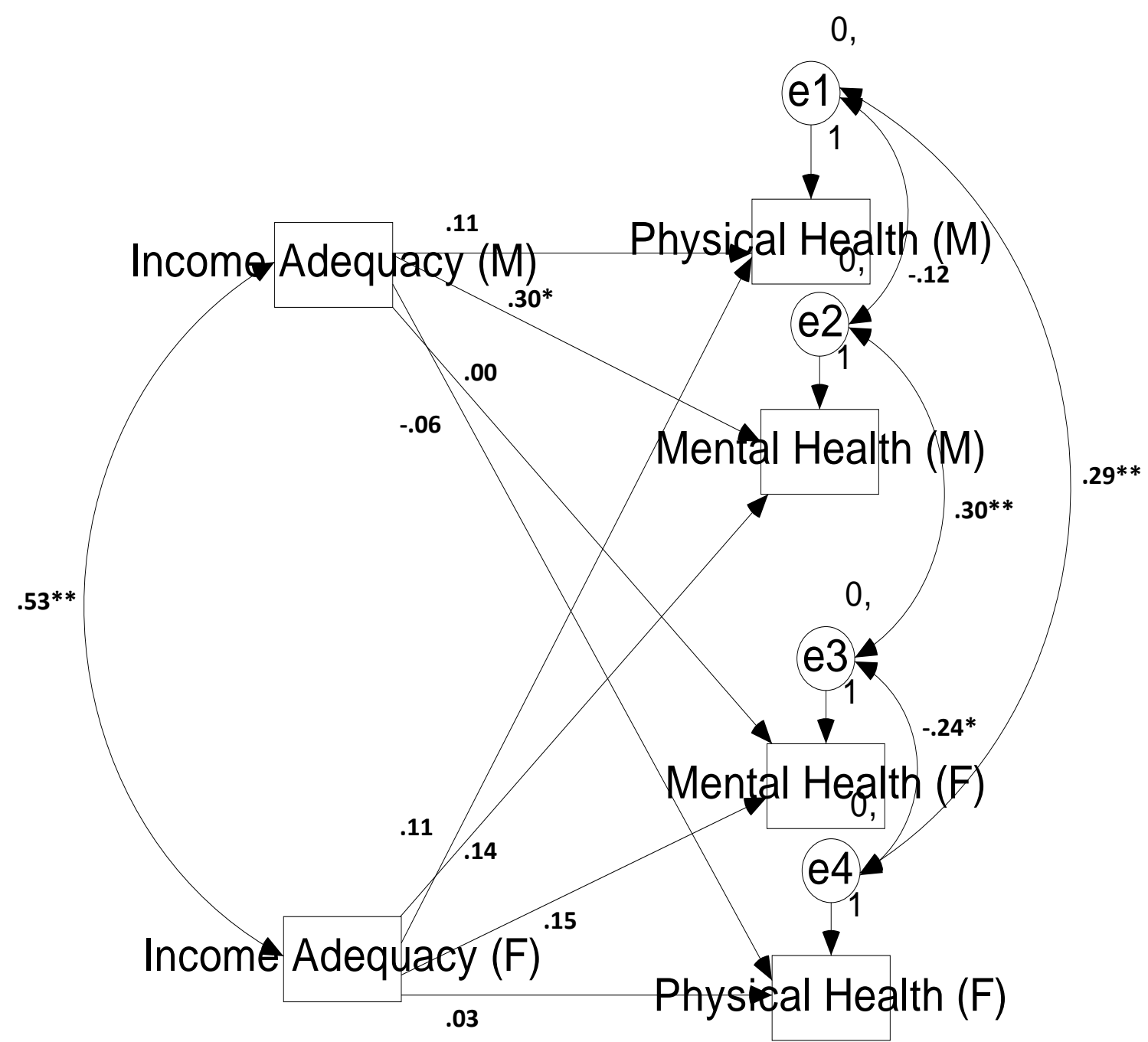

Note: $* * p<.01, * p<.05 . \mathrm{M}=$ scale measurement for Males; $\mathrm{F}=$ scale measurement for Females. 
Figure 6. Post-Hoc APIM 1.

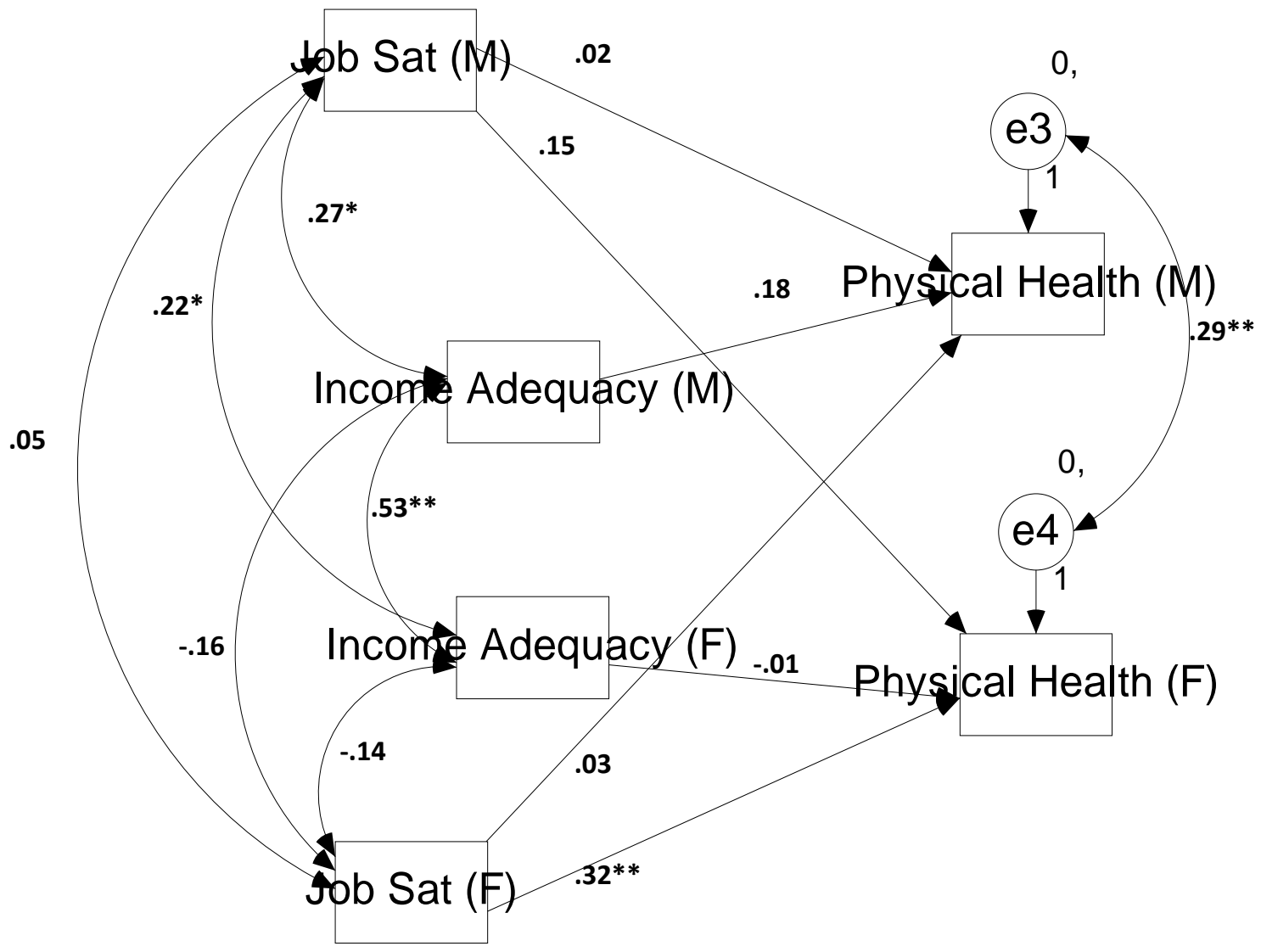

Note: $* * p<.01, * p<.05$. Job Sat $=$ Job Satisfaction scale. $\mathrm{M}=$ scale measurement for Males; $\mathrm{F}=$ scale measurement for Females. 
Figure 7. Post-Hoc APIM 2.

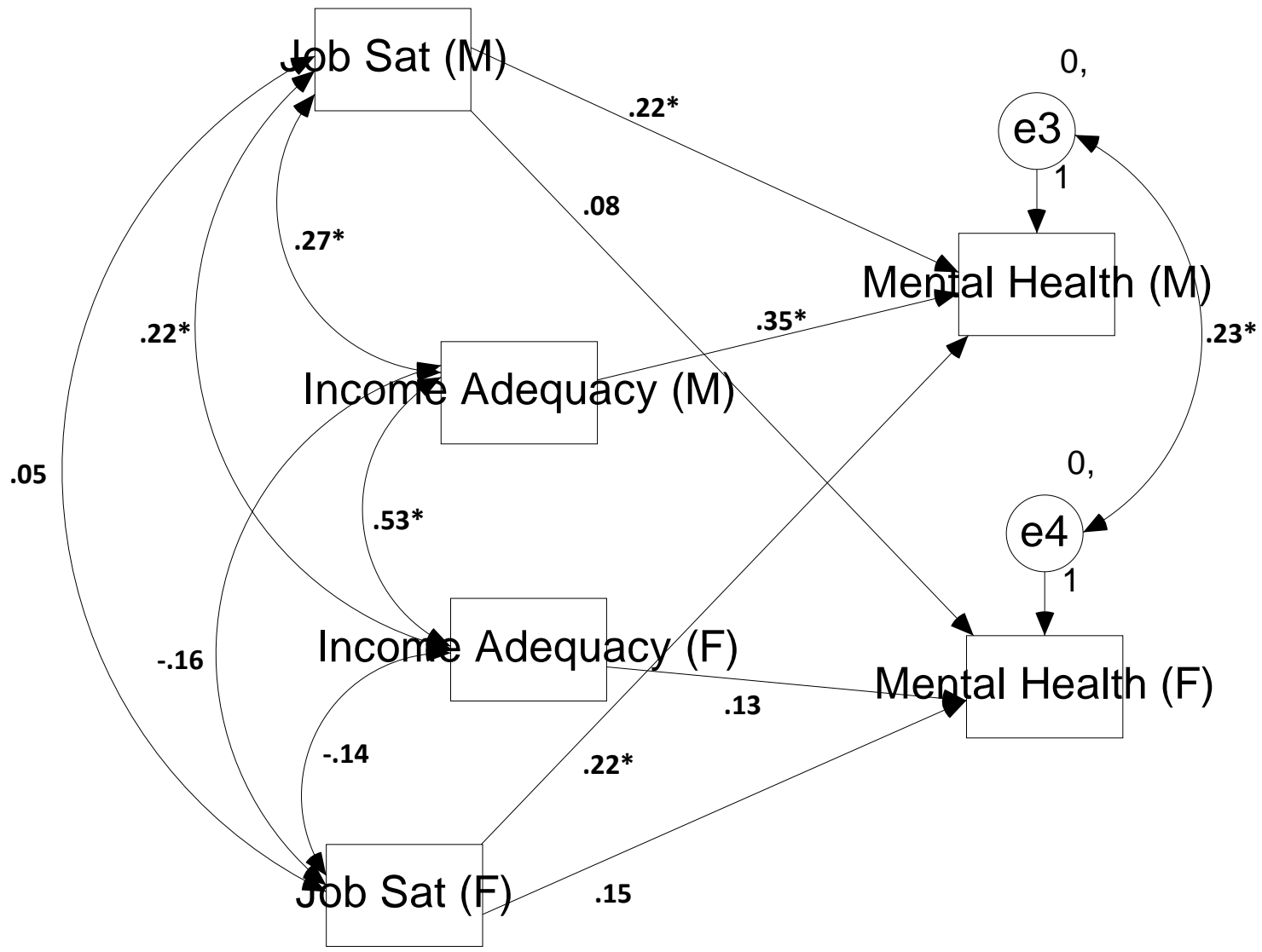

Note: $* * p<.01, * p<.05$. Job Sat $=$ Job Satisfaction scale. $\mathrm{M}=$ scale measurement for Males; F = scale measurement for Females. 


\section{References}

Ajzen, I., \& Fishbein, M. (1977). Attitude-behavior relations: A theoretical analysis and review of empirical research. Psychological Bulletin, 84, 888-918.

Allen, T. D., \& Armstrong, J. (2006). Further examination of the link between workfamily conflict and physical health: The role of health-related behavior. American Behavioral Scientist, 49, 1204-1221.

Bakker, A. B. (2005). Flow among music teachers and their students: The crossover of peak experience. Journal of Vocational Behavior, 66, 26-44.

Bakker, A. B., \& Demerouti, E. (2009). The crossover of work engagement between working couples: A closer look at the role of empathy. Journal of Managerial Psychology, 24, 220-236.

Bakker, A. B., Demerouti, E., \& Burke, R. (2009). Workaholism and relationship quality: A spillover-crossover perspective. Journal of Occupational Health Psychology, 14, 23-33.

Bakker, A. B., Demerouti, E., \& Dollard, M. F. (2008). How job demands affect partners' experience of exhaustion: Integrating work-family conflict and crossover theory. Journal of Applied Psychology, 93, 901-911.

Bakker, A. B., Demerouti, E., \& Schaufeli, W. B. (2005). The crossover of burnout and work engagement among working couples. Human Relations, 58, 661-689.

Bakker, A. B., LeBlanc, P. M., \& Schaufeli, W. B. (2005). Burnout contagion among intensive care nurses. Journal of Advanced Nursing, 51, 276-287. 
Bakker, A. B., \& Schaufeli, W. B. (2000). Burnout contagion processes among teachers. Journal of Applied Social Psychology, 30, 2289-2308.

Bakker, A. B., Schaufeli, W. B., Sixma, H., \& Bosveld, W. (2001). Burnout contagion among general practitioners. Journal of Social and Clinical Psychology, 20, 8298.

Bakker, A. B., Van Emmerik, H., \& Euwema, M. C. (2006). Crossover of burnout and engagement in work teams. Work and Occupations, 33, 464-489.

Bakker, A. B., Westman, M., \& Schaufeli, W. B. (2007). Crossover of burnout: An experimental design. European Journal of Work and Organizational Psychology, 16, 220-239.

Bakker, A. B., Westman, M. \& van Emmerik, I. J. (2009). Advancements in crossover theory. Journal of Managerial Psychology, 24, 206-219.

Bakker, A. B., \& Danthopoulou, D. (2009). The crossover of daily work engagement: Test of an Actor-Partner Interdependence Model. Journal of Applied Psychology, 94, 1562-1571.

Barnett, R. C., Gareis, K. C., \& Brennan, R. T. (2008). Wives' shift work schedules and husbands' and wives' well-being in dual-earner couples with children: A withincouple analysis. Journal of Family Issues, 29, 396-422.

Barnett, R. C., Raudenbush, S. W., Brennan, R. T., Pleck, J. H., \& Marshall, N. L. (1995). Changes in job and marital experience and change in psychological distress: A longitudinal study of dual-earner couples. Journal of Personality and Social Psychology, 69, 839-850. 
Beehr, T. A. \& Glazer, S. (2005). Organizational role stress. In J. Barling, E. K. Kelloway, \& M. R. Frone (Eds.), Handbook of work stress (pp. 7-33). Thousand Oaks, CA: SAGE Publications.

Beehr, T. A., Johnson, L. B., \& Nieva, R. (1995). Occupational stress: Coping of police and their spouses. Journal of Organizational Psychology, 16, 3-25.

Begley, T. M., \& Czajka, J. M. (1993). Panel analysis of the moderating effects of commitment on job satisfaction, intent to quit, and health following organizational change. Journal of Applied Psychology, 78, 552-556.

Bellavia, G. M., \& Frone, M. R. (2005). Work-family confict. In J. Barling, E. K. Kelloway, \& M. R. Frone (Eds.), Handbook of work stress (pp. 113-147). Thousand Oaks, CA: SAGE Publications.

Belkic, K. L., Landsbergis, P. A., Schall, P. L., \& Baker, D. (2004). Is job strain a major source of cardiovascular disease risk? Scandinavian Journal of Work, Environment and Health, 30, 85-128.

Bolger, N., DeLongis, A., Kessler, R., \& Wethington, E. (1989). The contagion of stress across multiple roles. Journal of Marriage and the Family, 51, 175-183.

Bond, J. T., Thompson, C., Galinsky, E. \& Prottas, D. (2002). Highlights of the national study of the changing workforce. New York, NY: Families and Work Institute.

Booth, A. (1977). Wife's employment and husband's stress: A replication and refutation. Journal of Marriage and the Family, 39, 645-650.

Bowling, A. (2005). Just one question: If one question works, why ask several? Journal of Epidemiology and Community Health, 59, 342-345. 
Brockwood, K. J., Hammer, L. B., Neal, M. B., \& Colton, C. L. (2001). Effects of accommodations made at home and at work on wives' and husbands' family and job satisfaction. Journal of Feminist Family Therapy, 13, 41-64.

Bronfenbrenner, U. (1977). Toward an experimental ecology of human development. American Psychologist, 32, 513-531.

Brunswick, E. (1952). The conceptual framework of psychology. Chicago: University of Chicago Press.

Bureau of Labor Statistics (2005). United States Department of Labor. Retrieved January 20, 2009, from, www.bls.gov

Bureau of Labor Statistics (2008). National Industry-Specific Occupational Employment and Wage Estimates. Retrieved June 3, 2009, from, www.bls.gov

Bureau of Labor Statistics (2009a). Highlights of women's earnings in 2008. United States Department of Labor. Retrieved April 4, 2010, from, http://www.bls.gov/cps/cpswom2008.pdf

Bureau of Labor Statistics (2009b). Monthly labor review November 2009. United States Department of Labor. Retrieved April 4, 2010, from, http://www.bls.gov/opub/mlr/2009/11/art5full.pdf

Bureau of Labor Statistics (2010). Career guide to industries, 2010-11 edition, grocery stores. United States Department of Labor. Retrieved April 4, 2010, from, http://www.bls.gov/oco/cg/cgs024.htm

Burke, R. J., Weir, T., \& Duwors, R. E. (1980). Work demands on administrators and spouse well being. Human Relations, 33, 253-278. 
Campbell, L., \& Kashy, D. A. (2002). Estimating actor, partner, and interaction effects for dyadic data using PROC MIXED and HLM: A user-friendly guide. Personal Relationships, 9, 327-342.

Chan, C. J. \& Margolin, G. (1994). The relationship between dual-earner couples' daily work mood and home affect. Journal of Social and Personal Relationships, 11, 573-586.

Cinamon, R. G., Weisel, A., \& Tzuk, K. (2007). Work-family conflict within the family. Journal of Career Development, 34, 79-100.

Cook, W. L., \& Kenny, D. A. (2005). The actor-partner interdependence model: A model of bidirectional effects in developmental studies. International Journal of Behavioral Development, 29, 101-109.

Cronkite, R., \& Moos, R. (1984). The role of predisposing and moderating factors in the stress-illness relationship. Journal of Health and Social Behavior, 25, 372-393.

Cross, S. E., \& Madson, L. (1997). Models of the self: Self-construals and gender. Psychological Bulletin, 122, 5-37.

Crossfield, S., Kinman, G., \& Jones, F. (2005). Crossover of occupational stress in dualcareer couples: The role of work demands and supports, job commitment and marital communication. Community, Work \& Family, 8, 211-232.

de Jonge, J., Dollard, M. F., Dormann, C., Le Blanc, P. M., \& Houtman, I. L. (2000). The demand-control model: Specific demands, specific control and a welldefined group. International Journal of Stress Management, 7, 269-287. 
de Lange, A. H., Taris, T. W., Kompier, M. A., Houtman, I. L., \& Bongers, P. M. (2003). Journal of Occupational Health Psychology, 8, 282-305.

Demerouti, E., Bakker, A. B., \& Schaefeli, W. B. (2005). Spillover and crossover of exhaustion and life satisfaction among dual-earner parents. Journal of Vocational Behavior, 67, 266-289.

Department of Health and Human Services (2009). Annual update of the HHS poverty guidelines. Federal Register, 74, 4199-4201.

Dikkers, J. S., Geurts, S. A., Kinnunen, U., Kompier, M. A., \& Taris, T. W. (2007). Crossover between work and home in dyadic partner relationships. Scandinavian Journal of Psychology, 48, 529-538.

Eckenrode, J. \& Gore, S. (1981). Stressful events and social support: The significance of context. In B. Gottlieb (Ed.), Social networks and social support (pp. 43-68). Beverly Hills, CA: Sage

Elovainio, M., Kivimaki, M. \& Vahtera, J. (2002). Organizational justice: Evidence of a new psychosocial predictor of health. American Journal of Public Health: Research and Practice, 92, 105-108.

Elsass, P. M., \& Veiga, J. F. (1997). Job control and job strain: A test of three models. Journal of Occupational Health Psychology, 2, 195-211.

Etzion, D. \& Westman, M. (2001). Job stress, vacation, and the crossover of strain between spouses - stopping the vicious cycle. Man and Work, 11, 106-118.

Families and Work Institute (2006). What do we know about entry-level, hourly employees? New York, NY: Families and Work Institute. 
Faragher, E. B., Cass, M., \& Cooper, C. L. (2005). The relationship between job satisfaction and health: A meta-analysis. Occupational Environmental Medicine, 62, 105-112.

Fletcher, B. (1983). Marital relationships as a cause of death: An analysis of occupational mortality and the hidden consequences of marriage - some U.K. data. Human Relations, 36, 123-124.

Fletcher, B. (1988). Occupation, marriage, and disease specific mortality concordance. Social Science \& Medicine, 27, 615-622.

Fox, M. L., Dwyer, D. J., \& Ganster, D. C. (1993). Effects of stressful job demands and control on physiological and attitudinal outcomes in a hospital setting. Academy of Management Journal, 36, 289-318.

Fredrickson, B. L. (2001). The role of positive emotions in positive psychology: The broaden-and-build theory of positive emotions. American Psychology, 56, 218226.

Frese, M., \& Zapf, D. (1994). Action as the core of work psychology: A German approach. In H. C. Triandis, M. D. Dunnette, \& L. M. Hough (Eds.), Handbook of industrial and Organizational psychology (Vol. 4, pp. 271-340). Palo Alto, CA: Consulting Psychologists Press.

Fried, Y., \& Ferris, G. R. (1987). The validity of the job characteristics model: A review and meta-analysis. Personnel Psychology, 40, 287-322. 
Frone, M. R. (2003). Work-Family Balance. In J. C. Quick \& L. E. Tetrick (Eds.), Handbook of Occupational Health Psychology (pp. 143-162). Washington, D.C.: American Psychological Association.

Frone, M. R., Russell, M., \& Cooper, M. L. (1997). Relation of work-family conflict to health outcomes: A four-year longitudinal study of employed parents. Journal of Occupational and Organizational Psychology, 70, 325-335.

Ganster, D. C. (1989). Worker control and well-being: A review of research in the workplace. In S. L. Sauter, J. J. Hurrell, \& C. L. Cooper (Eds.), Job Control and Worker Health (pp. 3-24). Chichester: Wiley.

Ganster, D. C., \& Fusilier, M. R. (1989). Control in the workplace. In C. L. Cooper, \& I. T. Robertson (Eds.), International review of industrial and organizational psychology (pp. 235-280). Chichester: Wiley \& Sons.

Gareis, K. C., Barnett, R. C., \& Brennan, R. T. (2003). Individual and crossover effects of work schedule fit: A within couple analysis. Journal of Marriage and Family, $65,1041-1054$.

Gill, S. C., Butterworth, P., Rogers, B. \& MacKinnon, A. (2007). Validity of the mental health component scale of the 12-item short-form health survey (MCS-12) as measure of common mental disorders in the general population. Psychiatry Research, 152, 63-71.

Gorgievski-Duijvesteijn, M. J., Giesen, C. W., \& Bakker, A. B. (2000). Financial problems and health complaints among farm couples: Results of a 10-year follow-up study. Journal of Occupational Health Psychology, 5, 359-373. 
Grandey, A. A. (2003). When "the show must go on": Surface acting and deep acting as determinants of emotional exhaustion and peer-rated service delivery. Academy of Management Journal, 46, 86-96.

Green, S. G., Bull Shaefer, R. A., MacDermid, S. M., \& Weiss, H. M. (2010). Partner reactions to work-family conflict: Cognitive appraisal and indirect crossover in couples. Journal of Management, FORTHCOMING.

Greenberg, J. (2004). Stress fairness to fare no stress: Managing workplace stress by promoting organizational justice. Organizational Dynamics, 33, 352-365.

Greenhaus, J. H., \& Parasuraman, S. (1987). A work-nonwork interactive prospective of stress consequences. Journal of Organizational Behavior Management, 8, 3760.

Greenhaus, J. H., \& Powell, G. N. (2006). When work and family are allies: A theory of work-family enrichment. Academy of Management Review, 31, 72-92.

Griffin, J. M., Greiner, B. A., Stansfeld, S. A., \& Marmot, M. (2007). The effect of selfreported and observed job conditions on depression and anxiety conditions: A comparison of theoretical models. Journal of Occupational Health Psychology, 12, 334-349.

Hackman, J. R., \& Oldham, G. R. (1976). Motivation through the design of work: Test of a theory. Organizational Behavior and Human Performance, 16, 250-279.

Haines, V. Y., Marchand, A., \& Harvey, S. (2006). Crossover of workplace aggression experiences in dual-earner couples. Journal of Occupational Health Psychology, $11,305-314$. 
Hammer, L. B., Allen, E., \& Grigsby, T. (1997). Work-family conflict in dual-earner couples: Within-individual and crossover effects of work and family. Journal of Vocational Behavior, 50, 185-203.

Hammer, L. B., Bauer, T. N., \& Grandey, A. A. (2003). Work-family conflict and work-related withdrawal behaviors. Journal of Business and Psychology, 17, 419-436.

Hammer, L. B., Cullen, J. C., Neal, M. B., Sinclair, R. R. \& Shafiro, M. V. (2005a). The longitudinal effects of work-family conflict and positive spillover on depressive symptoms among dual-earner couples. Journal of Occupational Health Psychology, 10, 138-154.

Hammer, L. B., Kossek, E. E., Yragui, N. L., Bodner, T. E., \& Hanson, G. C. (2009). Development and validation of a multidimensional measure of family supportive supervisor behaviors (FSSB). Journal of Management, 35, 837-856.

Hammer, L. B., Neal, M. B., Newsom, J. T., Brockwood, K. J., \& Colton, C. L. (2005b). A longitudinal study of the effects of dual-earner couples' utilization of family-friendly workplace supports on work and family outcomes. Journal of Applied Psychology, 90, 799-810.

Hanson, B. G. (1995). General systems theory beginning with wholes. Washington, D.C.: Taylor and Francis.

Hartel, C. E., \& Page, K. M. (2009). Discrete emotional crossover in the workplace: The role of affect intensity. Journal of Managerial Psychology, 24, 237-253. 
Hatfield, E., Cacioppo, J., \& Rapson, R. (1994). Emotional contagion. New York: Cambridge University Press.

Haynes, S. G., Eaker, E. D., Feinleib, M. (1983). Spouse behavior and coronary heart disease in men: Prospective results from the Framingham heart study. American Journal of Epidemiology, 118, 1-22.

Heymann, J. (2006). Forgotten families: Ending the growing crisis confronting children and working parents in the global economy. New York, NY: Oxford University Press.

Holman, D. J., \& Wall, T. D. (2002). Work characteristics, learning-related outcomes, and strain: A test of competing direct effects, mediated, and moderated models. Journal of Occupational Health Psychology, 7, 283-301.

Howe, G. W., Levy, M. L., \& Caplan, R. D. (2004). Job loss and depressive symptoms in couples: Common stressors, stress transmission, or relationship disruption? Journal of Family Psychology, 18, 639-650.

Hu, L., \& Bentler, P. M. (1999). Cutoff criteria for fit indexes in covariance structure analysis: Conventional criteria versus new alternatives. Structural Equation Modeling, 6, 1-55.

Hu, Y., Stewart-Brown, S., Twigg, L., \& Weich, S. (2007). Can the 12-item general health questionnaire be used to measure positive mental health? Psychological Medicine, 37, 1005-1013.

Jackson, S. E., \& Maslach, C. (1982). After-effects of job-related stress: Families as victims. Journal of Occupational Behavior, 3, 63-77. 
Jackson, S. E., Zebeck, S., \& Summers, E. (1985). Family life disruptions: Effects of job-induced structural and emotional interference. Academy of Management Journal, 28, 574-586.

Jex, S. M., \& Beehr, T. A. (1991). Emerging theoretical and methodological issues in the study of work-related stress. Research in Personnel and Human Resources Management, 9, 311-365.

Jex, S. M., \& Crossley, C. D. (2005). Organizational consequences. In J. Barling, E. K. Kellowy, \& M. R. Frone (Eds.), Handbook of work stress (pp. 575-599). Thousand Oaks, CA: SAGE Publications.

Job Content Questionnaire Center (2009). The Job Content Questionnaire (JCQ). Retrieved May 15, 2009, from www.jcqcenter.org

Johnson, J. V., \& Hall, E. M. (1988). Job strain, work place support, and cardiovascular disease: A cross-sectional study of a random sample of the Swedish working population. American Journal of Public Health, 78, 1336-1342.

Jones, F. \& Fletcher, B. (1993a). An empirical study of occupational stress transmission in working couples. Human Relations, 46, 881-902.

Jones, F. \& Fletcher, B. (1993b). Transmission of occupational stress: A study of daily fluctuations in work stress and strain and their impact on marital partners. In $\mathrm{H}$. Schroder, K. Rescke, M. Johnston, \& S. Maes (Eds.), Health Psychology: Potential in diversity (pp. 328-338). Regensburg: Roderer Verlag.

Karasek, R. A. (1979). Job demands, job decision latitude, and mental strain: Implications for job redesign. Administrative Science Quarterly, 24, 285-308. 
Karasek, R. A. (1985). Job Content Instrument: Questionnaire and User's Guide. Los Angeles; University of South Carolina.

Karasek, R. A. (1989). Control in the workplace and its health-related aspects. In S. L. Sauter, J. J. Hurrell, \& C. L. Cooper (Eds.), Job Control and Worker Health (pp. 129-160). Chichester: Wiley.

Karasek, R. A., Brisson, C., Kawakami, N., Houtman, I., Bongers, P., \& Amick, B. (1998). The Job Content Questionnaire (JCQ): An instrument for internationally comparative assessments of psychosocial job characteristics. Journal of Occupational Health Psychology, 3, 322-355.

Karasek, R. A., \& Theorell, T. (1990). Healthy work: stress, productivity, and the reconstruction of working life. New York, NY: Basic Books.

Kashy, D. A., \& Kenny, D. A. (2000). The analysis of data from dyads and groups. In H. T. Reis \& C. M. Judd (Eds.), Handbook of research methods in social psychology (pp. 451-477). New York: Cambridge University Press.

Katz, J., Beach, S. \& Joiner, T. (1999). Contagious depression in dating couples. Journal of Social and Clinical Psychology, 18, 1-13.

Katz, J., Monnier, J., Libet, J., Shaw, D., \& Beach, S. R. (2000). Individual and crossover effects of stress on adjustment in medical student marriages. Journal of Marital and Family Therapy, 26, 341-351.

Kelloway, E. K., Sivanathan, N., Francis, L., \& Barling, J. (2005). Poor leadership. In J. Barling, E. K. Kelloway, \& M. R. Frone (Eds.), Handbook of work stress (pp. 89-112). Thousand Oaks, CA: SAGE Publications. 
Kenny, D. A., \& Judd, C. M. (1984). Estimating the nonlinear and interactive effects of latent variables. Psychological Bulletin, 96, 201-210.

Kenny, D. A., Kashy, D. A., \& Cook, W. L. (2006). Dyadic data analysis. New York: Guilford Press.

Kessler, R. C., \& McLeod, J. D. (1984). Sex differences in vulnerability to undesirable life events. American Sociological Review, 49, 620-631.

Kiecolt-Glaser, J. K., McGuire, L., Robles, T., \& Glaser, R. (2002).

Psychoneuroimmunology: Psychological influences on immune function and health. Journal of Consulting and Clinical Psychology, 70, 537-547.

Kivimaki, M., Elovainio, M., Vahtera, J., \& Ferrie, J. E. (2003). Organizational justice and health of employees: Prospective cohort study. Occupational and Environmental Medicine, 60, 27-33.

Kline, R. B. (2005). Principles and practice of structural equation modeling: Second edition. New York, NY: The Guilford Press.

Kossek, E. E., Pichler, S. M., Meece, D., \& Barratt, M. E. (2008). Family, friend, and neighbor child care providers and maternal well-being in low-income systems: An ecological social perspective. Journal of Occupational and Organizational Psychology, 81, 369-391.

Lambert, S. J. (2008). Passing the buck: Labor flexibility practices that transfer risk onto hourly workers. Human Relations, 61, 1203-1227.

Lambert, S. J., \& Henly, J. R. (2009). Scheduling in hourly jobs: Promising practices for the twenty-first century economy. Washington, D.C.: The Mobility Agenda. 
Landsbergis, P., \& Theorell, T. (2000). Measurement of psychosocial workplace exposure variables. Occupational Medicine: State of the Art Reviews, 15(1), 163-189.

Lavee, Y., \& Ben-Ari, A. (2007). Relationship of dyadic closeness with work-related stress: A daily diary study. Journal of Marriage and Family, 69, 1021-1035.

Lazarus, R. S. (1991). Emotion and adaptation. NewYork: Oxford.

Lerner, D. J., Levine, S., Malspeis, S., D’Agostino, R. B. (1994). Job strain and healthrelated quality of life in a national sample. American Journal of Public Health, $84,1580-1585$.

Lim, S., Cortina, L. M., \& Magley, V. J. (2008). Personal and workgroup incivility: Impact on work and health outcomes. Journal of Applied Psychology, 93, 97107.

Long, N. R., \& Voges, K. E. (1987). Can wives perceive the source of their husbands' occupational stress? Journal of Occupational Psychology, 60, 235-242.

Matthews, R. A., Del Priore, R. E., Acitelli, L. K., \& Barnes-Farrell, J. L. (2006). Work-to-relationship conflict: Crossover effects in dual-earner couples. Journal of Occupational Health Psychology, 11, 228-240.

McClelland, G. H., \& Judd, C. M. (1993). Statistical Difficulties of Detecting Interactions and Moderator Effects. Psychological Bulletin, 114, 376-390.

Meier, L. L., Semmer, N. K., Elfering, A., \& Jacobshagen, N. (2008). The double meaning of control: Three way interactions between internal resources, job 
control, and stressors at work. Journal of Occupational Health Psychology, 13, 244-258.

Mills, R. T., Grasmick, H. G., Morgan, C. S., \& Wenk, D. (1992). The effects of gender, family satisfaction, and economic strain on psychological well-being. Family Relations, 41, 440-445.

Mitchell, R., Cronkite, R., \& Moos, R. (1983). Stress, coping and depression among married couples. Journal of Abnormal Psychology, 92, 433-448.

Morgeson, F. P., \& Campion, M. A. (2003). Work design. In W. C. Borman, D. R. Ilgen, \& R. J. Klimoski (Eds.), Handbook of Psychology: Industrial and Organizational Psychology (Vol. 12, pp. 423-452). Hoboken, NJ: John Wiley \& Sons.

Morrison, D., \& Clements, R. (1997). The effects of one partner's job characteristics on the other partner's distress: A serendipitous, but naturalistic, experiment. Journal of Occupational and Organizational Psychology, 70, 307-324.

Myers, J. L. (1979). Fundamentals of experimental design ( $3^{\text {rd }}$ ed.). Boston: Allyn \& Bacon.

Nunnally, J. (1978). Psychometric theory. New York: McGraw-Hill.

Olivius, G., Ostergren, P., Hanson, B., \& Lyttkens, C. H. (2004). Parental economic stress: Evidence of an overlooked public health risk among Swedish families. The European Journal of Public Health, 14, 354. 
Parasuraman, S., Greenhaus, J. H., \& Granrose, C. S. (1992). Role stressors, social support and well-being among two-career couples. Journal of Organizational Behavior, 13, 339-356.

Parkes, K. R., Mendham, C. A., \& Von Rabenau, C. (1994). Social support and the demand-discretion model of job stress: Tests of additive and interactive effects in two samples. Journal of Vocational Behavior, 44, 91-113.

Pavett, C. M. (1986). High-stress professions: Satisfaction, stress, and well-being of spouses of professionals. Human Relations, 39, 1141-1154.

Pearlin, L., Lieberman, M., Menaghan, E., \& Mullan, J. (1981). The stress process. Journal of Health and Social Behavior, 22, 337-356.

Pearson, C. M., \& Porath, C. L. (2005). On the nature, consequences, and remedies Of workplace incivility: No time for "nice"? Think again. Academy of Management Executive, 19, 7-18.

Ping, R. A. (1996). Latent variable interaction and quadratic effect estimation: A twostep technique using structural equation analysis. Psychological Bulletin, 119, $166-175$.

Plunkett Research, Ltd. (2009). Plunkett's Food, Beverage and Tobacco Industry. Retrieved March 10, 2009, from http://www.plunkettresearch.com/Industries/FoodBeverageTobacco/tabid/203/ Default.aspx\#IndustryTrends

Price, R. H., Choi, J. M., \& Vinokur, A. D. (2002). Links in the chain of adversity following job loss: How financial strain and loss of personal control lead to 
depression, impaired functioning, and poor health. Journal of Occupational Health Psychology, 7, 302-312.

Prince, M., Manolis, C., \& Minetor, R. (2007). Life satisfaction crossover among couples. In R. J. Esten (Ed.), Advancing Quality of Life in a Turbulant World (pp. 191-208), Dordrecht, The Netherlands: Springer.

Probst, T. (2004). Economic stressors. In J. Barling, E. Kelloway \& M. Frone (Eds.), Handbook of Work Stress (pp. 267-297). Thousand Oaks, CA: SAGE Publications.

Quick, J. C., Quick, J. D., Nelson, D. L., \& Hurrell, J. J. (1997). Stress in organizations. In J. C. Quick, J. D. Quick, D. L. Nelson \& J. J. Hurrell (Eds.), Preventative Stress Management in Organizations (pp. 1-20). Washington, D. C.: American Psychological Association.

Richardson, K. M., \& Rothstein, H. R. (2008). Effects of occupational stress management intervention programs: A meta-analysis. Journal of Occupational Health Psychology, 13, 69-93.

Riese, H., Van Doornen, L. J., Houtman, I. L., \& Geus, E. J. (2000). Job strain and risk indicators for cardiovascular disease in young female nurses. Health Psychology, 19, 429-440.

Riley, D., \& Eckenrode, J. (1986). Social ties: Costs and benefits within different subgroups. Journal of Personality and Social Psychology, 51, 770-778.

Roberts, R., \& O’Keefe, S. J. (1981). Sex differences in depression re-examined. Journal of Health and Social Behavior, 22, 394-400. 
Rook, S. K., Dooley, D., \& Catalano, R. (1991). Stress transmission: The effects of husbands' job stressors on emotional health of their wives. Journal of Marriage and the Family, 53, 165-177.

Rosenfield, S. (1980). Sex differences in depression: Do women always have higher rates? Journal of Health and Social Behavior, 21, 33-42.

Rosenfield, S. (1992). The cost of sharing: Wives' employment and husbands' mental health. Journal of Health and Social Behavior, 33, 213-225.

Rospenda, K. M., Richman, J. A., Ehmke, J. L., \& Zlatoper, K. W. (2005). Is workplace harassment hazardous to your health? Journal of Business and Psychology, 20, 95-110.

Rowe, R., \& Snizek, W. E. (1995). Gender differences in work values: Perpetuating the myth. Work and Occupations, 22, 215-229.

Sanne, B., Mykletun, A., Dahl, A. A., Moen, B. E., \& Tell, G. S. (2005). Testing the job-demand-control-support model with anxiety and depression as outcomes: The Hordaland Health Study. Occupational Medicine, 55, 463-473.

Schnall, P. L., Landsbergis, P. A., \& Baker, D. (1994). Job strain and cardiovascular disease. Annual Review of Public Health, 15, 381-411.

Schaufeli, W. B., \& Bakker, A. B. (2004). Job demands, job resources, and their relationship with burnout and engagement: A multi sample study. Journal of Organizational Behavior, 25, 293-315. 
Schaufeli, W. B., Salanova, M., Gonzalez-Roma, V., \& Bakker, A. B. (2002). The measurement of engagement and burnout: A confirmative analytic approach. Journal of Happiness Studies, 3, 71-92.

Sears, L. E. (2008). Work-related outcomes of financial stress: Relating perceived income adequacy and financial strain to job performance and worker wellbeing. Unpublished master's thesis, Portland State University.

Segerstrom, S. C. and Miller, G. E. (2004). Psychological Stress and the Human Immune System: A Meta-Analytic Study of 30 Years of Inquiry. Psychological Bulletin, 130, 601-630.

Sonnetag, S. \& Frese, M. (2003). Stress in organizations. In W. C. Borman, D. R. Ilgen, \& R. J. Klimoski (Eds.), Comprehensive handbook of psychology, Volume 12: Industrial and organizational psychology (pp. 453-491). Hoboken: Wiley.

Staines, G. L., Pottic, K. G., \& Fudge, D. A. (1986). Wives' employment and husbands' attitudes toward work and life satisfaction. Journal of Applied Psychology, 71, 118-128.

Stangor, C. (1998). Research Methods for the Behavioral Sciences. Boston: Houghton Mifflin.

Stansfeld, S. A., Bosma, H., Hemingway, H., \& Marmot, M. G. (1998). Psychosocial work characteristics and social support as predictors of SF-36 health functioning: The Whitehall II study. Psychosomatic Medicine, 60, 247-255. 
Stewart, B. \& Archbold, P. (1996). Oregon Family Caregiving Survey. Unpublished manuscript. Portland, OR: Oregon Health Sciences University, School of Nursing.

Stoll, K. (2005). Paying a premium: The added cost of care for the uninsured. New York: Families USA.

Sutherland, V. \& Cooper, C. (2000). Strategic stress management: An organizational approach. London: MacMillan Business.

Takeuchi, R., Yun, S., \& Tesluk, P. E. (2002). An examination of crossover and spillover effects of spousal and expatriate cross-cultural adjustment on expatriate outcomes. Journal of Applied Psychology, 4, 655-666.

Taris, T. W., Kalimo, R., \& Shaufeli, W. B. (2002). Inequity at work: Its measurement and association with worker health. Work and Stress, 16, 287-301.

Taylor, F. W. (1911). The principles of scientific management. New York: W. W. Norton.

Taris, T. W. (2006). Bricks without clay: On urban myths in occupational health psychology. Work \& Stress, 20, 99-104.

Tepper, B. J. (2001). Health consequences of organization injustice: Tests of main and interactive effects. Organizational Behavior and Human Decision Processes, $86,197-215$.

Theorell, T. (2003). To be able to exert control over one's situation: A necessary condition for coping with stressors. In J. C. Quick \& L. E. Tetrick (Eds.), 
Handbook of Occupational Health Psychology (pp. 201-220). Washington, D. C.: American Psychological Association.

Theorell, T., \& Karasek, R. A. (1996). Current issues related to psychosocial job strain and cardiovascular disease research. Journal of Occupational Health Psychology, 1, 9-26.

Totterdell, P. (2005). Work schedules. In J. Barling, E. K. Kellowy, \& M. R. Frone (Eds.), Handbook of work stress (pp. 35-62). Thousand Oaks, CA: SAGE Publications.

Ullman, J. B. (1996). Structural equation modeling. In B. G. Tabachnik \& L. S. Fidell (Eds.), Using Multivariate Statistics ( $3^{\text {rd }}$ Ed.) (pp. 709-812). Northridge, CA: Harper Collins College Publishers.

van der Doef, M. \& Maes, S. (1998). The job demand-control(-support) model and physical health outcomes: A review of the strain and buffer hypothesis. Psychology \& Health, 13, 909-936.

van der Doef, M., \& Maes, S. (1999). The job demand-control(-support) model and psychological well-being: A review of 20 years of empirical research. Work \& Stress, 2, 87-114.

van der Doef, M., Maes, S., \& Diekstra, R. (2000). An examination of the job-controlsupport model with various occupational strain indicators. Anxiety, Stress, \& Coping, 13, 165-185. 
van Emmerik, I. J., \& Peeters, M. C. (2009). Crossover specificity of team-level workfamily conflict to individual-level work-family conflict. Journal of Managerial Psychology, 24, 254-268.

Vinokur, A., Price, R. H., \& Caplan, R. D. (1996). Hard times and hurtful partners: How financial strain affects depression and relationship satisfaction of unemployed persons and their spouses. Journal of Personality and Social Psychology, 71, 166-179.

Vinokur, A., \& van Ryn, M. (1993). Social support and undermining in close relationships: Their independent effects on mental health of unemployed persons. Journal of Personality and Social Psychology, 65, 350-359.

Vitaliano, P. T., Zhang, J., \& Scanlan, J. M. (2003). Is caregiving hazardous to one's physical health? A meta-anlaysis. Psychological Bulletin, 129, 946-972.

Waldron, T., Roberts, B., \& Reamer, A. (2004). Working hard, falling short: America's working families and the pursuit of economic security: A national report by the Working Poor Families Project, sponsored by the Annie E. Casey, Ford, and Rockefeller Foundations. Baltimore, MD: The Annie E. Casey Foundation

Wall, T. D., Jackson, P. R., Mularkey, S., \& Parker, S. K. (1996). The demands-control model of job strain: A more specific test. Journal of Occupational and Organizational Psychology, 69, 153-166.

Ware, J. E., Kosinski, M., \& Keller, S. D. (1996). A 12-item short-form health survey: Construction of scale and preliminary tests of reliability and validity. Medical Care, 34, 220-233. 
Warr, P. B. (1987). Work, unemployment and mental health. Oxford: Oxford University Press.

Warr, P. (2005). Work, well-being, and mental health. In J. Barling, E. K. Kellowy, \& M. R. Frone (Eds.), Handbook of work stress (pp. 547-573). Thousand Oaks, CA: SAGE Publications.

Watson, D. (1988). Intraindividual and interindividual analyses of positive and negative affect: Their relation to health complaints, perceived stress, and daily activities. Journal of Personality and Social Psychology, 54, 1020-1030.

Westman, M. (2001). Stress and strain crossover. Human Relations, 54, 557-591.

Westman, M, \& Etzion, D. (1995). Crossover of stress, strain, and resources from one spouse to another. Journal of Organizational Behavior, 16, 169-181.

Westman, M., \& Etzion, D. (1999). The crossover of strain from school principals to teachers and vice versa. Journal of Occupational Health Psychology, 4, 269278.

Westman, M., \& Etzion, D. (2005). The crossover of work-family conflict from one spouse to the other. Journal of Applied Social Psychology. 35, 1936-1957.

Westman, M., Etzion, D., \& Chen, S. (2009). Crossover of positive experiences from business travelers to their spouses. Journal of Managerial Psychology, 24, 269284.

Westman, M., Etzion, D., \& Danon, E. (2001). Job insecurity and crossover of burnout in married couples. Journal of Organizational Behavior, 22, 467-481. 
Westman, M., Etzion, D., \& Horovitz, S. (2004a).The toll of unemployment does not stop with the unemployed. Human Relations, 57, 823-844.

Westman, M., Keinan, G., Roziner, I., \& Benyamini, Y. (2008). The crossover of perceived health between spouses. Journal of Occupational Health Psychology, $13,168-180$.

Westman, M., \& Vinokur, A. D. (1998). Unraveling the relationship of distress levels within couples: Common stressors, empathetic reactions, or crossover via social interaction? Human Relations, 51, 137-156.

Westman, M., Vinokur, A. D., Hamilton, V. L., \& Roziner, I. (2004b). Crossover of marital dissatisfaction during military downsizing among Russian army officers and their spouses. Journal of Applied Psychology, 89, 769-779.

Xie, J. I. \& Johns, G. (1995). Job scope and stress: Can job scope be too high? Academy of Management Journal, 38, 1288-1309. 
Appendix A

Crossover Research

\begin{tabular}{|c|c|c|c|c|c|c|c|}
\hline Citation & Sample & $\begin{array}{l}\text { Type of } \\
\text { Crossover }\end{array}$ & Mechanism & $\begin{array}{l}\text { Origin/ } \\
\text { Destination }\end{array}$ & Crossover of & Directionality & Findings \\
\hline Bakker (2005) & $\begin{array}{l}178 \text { music } \\
\text { teachers and } \\
605 \text { students }\end{array}$ & $\begin{array}{l}\text { Resource } \rightarrow \\
\text { Resource }\end{array}$ & $\begin{array}{l}\text { Direct } \\
\text { Crossover }\end{array}$ & $\begin{array}{l}\text { Work } \rightarrow \\
\text { Work }\end{array}$ & $\begin{array}{l}\text { Flow (similar } \\
\text { to engagement } \\
\text { concept) }\end{array}$ & $\begin{array}{l}\text { Unidirectional } \\
\text { (Teacher to } \\
\text { student) }\end{array}$ & $\begin{array}{l}\text { The more flow experiences } \\
\text { music teachers reported, the } \\
\text { higher the frequency of } \\
\text { comparable experiences in } \\
\text { their students }\end{array}$ \\
\hline $\begin{array}{l}\text { Bakker \& } \\
\text { Demerouti } \\
(2009)\end{array}$ & $\begin{array}{l}175 \text { Dutch } \\
\text { dual-earner } \\
\text { couples }\end{array}$ & $\begin{array}{l}\text { Resource } \rightarrow \\
\text { Resource }\end{array}$ & $\begin{array}{l}\text { Direct } \\
\text { Crossover }\end{array}$ & $\begin{array}{l}\text { Work } \rightarrow \\
\text { Home }\end{array}$ & Engagement & $\begin{array}{l}\text { Unidirectional } \\
\text { (Women to } \\
\text { partners) }\end{array}$ & $\begin{array}{l}\text { Crossover of engagement } \\
\text { from working women to } \\
\text { their partners; men high in } \\
\text { perspective taking were } \\
\text { more strongly influenced by } \\
\text { their partners' work } \\
\text { engagement than their } \\
\text { counterparts (nonsignificant } \\
\text { for empathy). }\end{array}$ \\
\hline $\begin{array}{l}\text { Bakker, } \\
\text { Demerouti \& } \\
\text { Burke (2009) }\end{array}$ & $\begin{array}{l}168 \text { Dutch } \\
\text { dual-earner } \\
\text { couples }\end{array}$ & $\begin{array}{l}\text { Resource } \rightarrow \\
\text { Resource; } \\
\text { Stressor } \rightarrow \\
\text { Resource }\end{array}$ & $\begin{array}{l}\text { Direct } \\
\text { Crossover; } \\
\text { Indirect } \\
\text { Crossover }\end{array}$ & $\begin{array}{l}\text { Home } \rightarrow \\
\text { Home }\end{array}$ & $\begin{array}{l}\text { Relationship } \\
\text { Satisfaction; } \\
\text { Reduced } \\
\text { support to } \\
\text { relationship } \\
\text { satisfaction }\end{array}$ & $\begin{array}{l}\text { Bidirectional } \\
\text { (Partner to } \\
\text { partner) }\end{array}$ & $\begin{array}{l}\text { Direct crossover of } \\
\text { relationship satisfaction } \\
\text { between partners. } \\
\text { Workaholism was positively } \\
\text { related to work-family } \\
\text { conflict, leaving the partners } \\
\text { to feel less supported, } \\
\text { resulting in reduced } \\
\text { relationship satisfaction }\end{array}$ \\
\hline
\end{tabular}




\begin{tabular}{|c|c|c|c|c|c|c|c|}
\hline Citation & Sample & $\begin{array}{l}\text { Type of } \\
\text { Crossover }\end{array}$ & Mechanism & $\begin{array}{l}\text { Origin/ } \\
\text { Destination }\end{array}$ & Crossover of & Directionality & Findings \\
\hline & & & & & & & (indirect crossover). \\
\hline $\begin{array}{l}\text { Bakker, } \\
\text { Demerouti \& } \\
\text { Dollard (2008) }\end{array}$ & $\begin{array}{l}168 \text { Dutch } \\
\text { dual-earner } \\
\text { couples }\end{array}$ & $\begin{array}{l}\text { Stressor } \rightarrow \\
\text { Stressor; } \\
\text { Stressor } \rightarrow \\
\text { Stressor }\end{array}$ & $\begin{array}{l}\text { Direct } \\
\text { Crossover; } \\
\text { Indirect } \\
\text { Crossover }\end{array}$ & $\begin{array}{l}\text { Work } \rightarrow \\
\text { Home }\end{array}$ & $\begin{array}{l}\text { Work-to- } \\
\text { family conflict } \\
\text { on home } \\
\text { demands; } \\
\text { Social } \\
\text { undermining } \\
\text { on home } \\
\text { demands }\end{array}$ & $\begin{array}{l}\text { Bidirectional } \\
\text { (Partner to } \\
\text { partner) }\end{array}$ & $\begin{array}{l}\text { For both genders, job } \\
\text { demands (work overload and } \\
\text { emotional demands) were } \\
\text { related to their own reported } \\
\text { work-to-family conflict, } \\
\text { which in turn was related to } \\
\text { partner's home demands, } \\
\text { family-to-work conflict and } \\
\text { exhaustion. Social } \\
\text { undermining mediated the } \\
\text { relationship between } \\
\text { individuals' work-to-family } \\
\text { conflict and their partners' } \\
\text { home demands. }\end{array}$ \\
\hline $\begin{array}{l}\text { Bakker, } \\
\text { Demerouti \& } \\
\text { Schaufeli } \\
(2005)\end{array}$ & $\begin{array}{l}323 \text { couples } \\
\text { working in a } \\
\text { variety of } \\
\text { occupations }\end{array}$ & $\begin{array}{l}\text { Resource } \rightarrow \\
\text { Resource; } \\
\text { Strain } \rightarrow \\
\text { Strain }\end{array}$ & $\begin{array}{l}\text { Direct } \\
\text { Crossover; } \\
\text { Common } \\
\text { Stressors }\end{array}$ & $\begin{array}{l}\text { Work } \rightarrow \\
\text { Home }\end{array}$ & $\begin{array}{l}\text { Engagement; } \\
\text { Burnout }\end{array}$ & $\begin{array}{l}\text { Bidirectional } \\
\text { (Partner to } \\
\text { partner) }\end{array}$ & $\begin{array}{l}\text { Crossover of work } \\
\text { engagement (vigor and } \\
\text { dedication) and burnout } \\
\text { (exhaustion and cynicism) } \\
\text { among partners; controlled } \\
\text { for several demands and } \\
\text { resources at home in an } \\
\text { attempt to rule out common } \\
\text { stressors and resources as a } \\
\text { spurious cause of crossover }\end{array}$ \\
\hline $\begin{array}{l}\text { Bakker, } \\
\text { LeBlanc \& }\end{array}$ & $\begin{array}{l}1849 \text { intensive } \\
\text { care nurses }\end{array}$ & $\begin{array}{l}\text { Strain } \rightarrow \\
\text { Strain }\end{array}$ & $\begin{array}{l}\text { Direct } \\
\text { Crossover }\end{array}$ & $\begin{array}{l}\text { Work } \rightarrow \\
\text { Work }\end{array}$ & Burnout & $\begin{array}{l}\text { Bidirectional } \\
\text { (Nurse to }\end{array}$ & $\begin{array}{l}\text { Nurses who reported the } \\
\text { highest prevalence of }\end{array}$ \\
\hline
\end{tabular}




\begin{tabular}{|c|c|c|c|c|c|c|c|}
\hline Citation & Sample & $\begin{array}{l}\text { Type of } \\
\text { Crossover }\end{array}$ & Mechanism & $\begin{array}{l}\text { Origin/ } \\
\text { Destination }\end{array}$ & Crossover of & Directionality & Findings \\
\hline $\begin{array}{l}\text { Schaufeli } \\
(2005)\end{array}$ & $\begin{array}{l}\text { from } 12 \\
\text { European } \\
\text { countries }\end{array}$ & & & & & nurse) & $\begin{array}{l}\text { burnout among their } \\
\text { colleagues were most } \\
\text { likely to experience high } \\
\text { levels of burnout } \\
\text { themselves; perceived } \\
\text { burnout complaints among } \\
\text { colleagues had a positive, } \\
\text { independent impact on each } \\
\text { of the three } \\
\text { burnout dimensions }\end{array}$ \\
\hline $\begin{array}{l}\text { Bakker \& } \\
\text { Schaufeli } \\
(2000)\end{array}$ & $\begin{array}{l}154 \text { Dutch } \\
\text { high school } \\
\text { teachers }\end{array}$ & $\begin{array}{l}\text { Strain } \rightarrow \\
\text { Strain }\end{array}$ & $\begin{array}{l}\text { Direct } \\
\text { Crossover }\end{array}$ & $\begin{array}{l}\text { Work } \rightarrow \\
\text { Work }\end{array}$ & Burnout & $\begin{array}{l}\text { Bidirectional } \\
\text { (Teacher to } \\
\text { teacher) }\end{array}$ & $\begin{array}{l}\text { Prevalence of perceived } \\
\text { burnout among colleagues } \\
\text { was most strongly related to } \\
\text { individual teachers' burnout } \\
\text { (i.e., emotional exhaustion } \\
\text { and depersonalization), } \\
\text { when the teachers were } \\
\text { highly susceptible to the } \\
\text { emotions of others and when } \\
\text { they frequently } \\
\text { communicated } \\
\text { with each other about work- } \\
\text { related problems }\end{array}$ \\
\hline $\begin{array}{l}\text { Bakker, } \\
\text { Schaufeli, } \\
\text { Sixma \& } \\
\text { Bosveld } \\
(2001)\end{array}$ & $\begin{array}{l}507 \text { general } \\
\text { practitioners }\end{array}$ & $\begin{array}{l}\text { Strain } \rightarrow \\
\text { Strain }\end{array}$ & $\begin{array}{l}\text { Direct } \\
\text { Crossover }\end{array}$ & $\begin{array}{l}\text { Work } \rightarrow \\
\text { Work }\end{array}$ & Burnout & $\begin{array}{l}\text { Bidirectional } \\
\text { (General } \\
\text { practitioner to } \\
\text { general } \\
\text { practitioner) }\end{array}$ & $\begin{array}{l}\text { Susceptibility to the } \\
\text { emotions expressed by } \\
\text { others had a moderating } \\
\text { effect on the relationship } \\
\text { between perceived burnout }\end{array}$ \\
\hline
\end{tabular}




\begin{tabular}{|c|c|c|c|c|c|c|c|}
\hline Citation & Sample & $\begin{array}{l}\text { Type of } \\
\text { Crossover }\end{array}$ & Mechanism & $\begin{array}{l}\text { Origin/ } \\
\text { Destination }\end{array}$ & Crossover of & Directionality & Findings \\
\hline & & & & & & & $\begin{array}{l}\text { complaints among } \\
\text { colleagues and individual } \\
\text { GPs' emotional exhaustion }\end{array}$ \\
\hline $\begin{array}{l}\text { Bakker, van } \\
\text { Emmerik \& } \\
\text { Euwema } \\
(2006)\end{array}$ & $\begin{array}{l}2,229 \text { Royal } \\
\text { Dutch } \\
\text { constabulary } \\
\text { officers } \\
\text { working in } \\
\text { one of } 85 \\
\text { teams }\end{array}$ & $\begin{array}{l}\text { Strain } \rightarrow \\
\text { Strain; } \\
\text { Resource } \rightarrow \\
\text { Resource }\end{array}$ & $\begin{array}{l}\text { Direct } \\
\text { Crossover }\end{array}$ & $\begin{array}{l}\text { Work } \rightarrow \\
\text { Work }\end{array}$ & $\begin{array}{l}\text { Burnout and } \\
\text { engagement }\end{array}$ & $\begin{array}{l}\text { Unidirectional } \\
\text { (Team levels } \\
\text { to individual } \\
\text { team } \\
\text { members) }\end{array}$ & $\begin{array}{l}\text { Team level burnout and } \\
\text { work engagement were } \\
\text { related to individual team } \\
\text { members' burnout } \\
\text { (emotional exhaustion, } \\
\text { reduced person efficacy and } \\
\text { cynicism) and work } \\
\text { engagement (vigor, } \\
\text { dedication and absorption) }\end{array}$ \\
\hline $\begin{array}{l}\text { Bakker, } \\
\text { Westman \& } \\
\text { Schaufeli } \\
(2007)\end{array}$ & $\begin{array}{l}40 \text { teachers; } \\
101 \text { soldiers }\end{array}$ & $\begin{array}{l}\text { Strain } \rightarrow \\
\text { Strain }\end{array}$ & $\begin{array}{l}\text { Direct } \\
\text { Crossover }\end{array}$ & $\begin{array}{l}\text { Work } \rightarrow \\
\text { Work }\end{array}$ & Burnout & $\begin{array}{l}\text { Bidirectional } \\
\text { (Teachers to } \\
\text { colleague; } \\
\text { soldiers to } \\
\text { colleague) }\end{array}$ & $\begin{array}{l}\text { Direct crossover of burnout } \\
\text { via a process of empathy; } \\
\text { crossover of burnout is } \\
\text { moderated by similarity with } \\
\text { the stimulus person }\end{array}$ \\
\hline $\begin{array}{l}\text { Bakker \& } \\
\text { Xanthopoulou } \\
(2009)\end{array}$ & $\begin{array}{l}62 \text { dyads of } \\
\text { employees }\end{array}$ & $\begin{array}{l}\text { Resource } \rightarrow \\
\text { Resource }\end{array}$ & $\begin{array}{l}\text { Direct } \\
\text { Crossover }\end{array}$ & $\begin{array}{l}\text { Work } \rightarrow \\
\text { Work }\end{array}$ & Engagement & $\begin{array}{l}\text { Bidirectional } \\
\text { (colleague to } \\
\text { colleague) }\end{array}$ & $\begin{array}{l}\text { Results confirmed the } \\
\text { crossover of daily work } \\
\text { engagement but only on } \\
\text { days when employee dyads } \\
\text { interacted more frequently } \\
\text { than usual. }\end{array}$ \\
\hline $\begin{array}{l}\text { Barnett, } \\
\text { Raudenbush, } \\
\text { Brennan, }\end{array}$ & $\begin{array}{l}210 \text { dual- } \\
\text { earner couples }\end{array}$ & $\begin{array}{l}\text { Strain } \rightarrow \\
\text { Strain }\end{array}$ & $\begin{array}{l}\text { Direct } \\
\text { Crossover }\end{array}$ & $\begin{array}{l}\text { Home } \rightarrow \\
\text { Home }\end{array}$ & Distress & $\begin{array}{l}\text { Bidirectional } \\
\text { (Spouse to } \\
\text { spouse) }\end{array}$ & $\begin{array}{l}\text { Increase in distress over time } \\
\text { of one partner was mirrored } \\
\text { in the changes in distress of }\end{array}$ \\
\hline
\end{tabular}




\begin{tabular}{|c|c|c|c|c|c|c|c|}
\hline Citation & Sample & $\begin{array}{l}\text { Type of } \\
\text { Crossover }\end{array}$ & Mechanism & $\begin{array}{l}\text { Origin/ } \\
\text { Destination }\end{array}$ & Crossover of & Directionality & Findings \\
\hline $\begin{array}{l}\text { Pleck \& } \\
\text { Marshall } \\
(1995)\end{array}$ & & & & & & & the other \\
\hline $\begin{array}{l}\text { Barnett, } \\
\text { Gareis \& } \\
\text { Brennan } \\
(2008)\end{array}$ & $\begin{array}{l}55 \text { female } \\
\text { nurses and } \\
\text { their spouses } \\
\text { (dual-earner } \\
\text { couples) }\end{array}$ & $\begin{array}{l}\text { Stressor } \rightarrow \\
\text { Stressor }\end{array}$ & $\begin{array}{l}\text { Direct } \\
\text { Crossover }\end{array}$ & $\begin{array}{l}\text { Work } \rightarrow \\
\text { Home }\end{array}$ & $\begin{array}{l}\text { Work } \\
\text { variables } \\
\text { (shift, hours, } \\
\text { and interaction } \\
\text { between) to } \\
\text { work-family } \\
\text { conflict }\end{array}$ & $\begin{array}{l}\text { Unidirectional } \\
\text { (Wife to } \\
\text { husband) }\end{array}$ & $\begin{array}{l}\text { Men whose wives regularly } \\
\text { worked evenings (compared } \\
\text { to day shift), showed a trend } \\
\text { to report more work-family } \\
\text { conflict, but only if wives } \\
\text { worked long hours as well }\end{array}$ \\
\hline $\begin{array}{l}\text { Beehr, } \\
\text { Johnson \& } \\
\text { Nieva (1995) }\end{array}$ & $\begin{array}{l}177 \text { male and } \\
\text { female police } \\
\text { officers and } \\
\text { their spouses }\end{array}$ & $\begin{array}{l}\text { Resource } \rightarrow \\
\text { Strain }\end{array}$ & $\begin{array}{l}\text { Direct } \\
\text { Crossover }\end{array}$ & $\begin{array}{l}\text { Work } \rightarrow \\
\text { Home }\end{array}$ & $\begin{array}{l}\text { Coping } \\
\text { strategies to } \\
\text { well-being }\end{array}$ & $\begin{array}{l}\text { Unidirectional } \\
\text { (Police } \\
\text { officers to } \\
\text { spouses) }\end{array}$ & $\begin{array}{l}\text { Police officers' coping } \\
\text { strategies were positively } \\
\text { related to spouses' well- } \\
\text { being }\end{array}$ \\
\hline $\begin{array}{l}\text { Bolger } \\
\text { DeLongis, } \\
\text { Kessler \& } \\
\text { Wethington } \\
\text { (1989) }\end{array}$ & $\begin{array}{l}166 \text { married } \\
\text { couples }\end{array}$ & $\begin{array}{l}\text { Stressor } \rightarrow \\
\text { Stressor }\end{array}$ & $\begin{array}{l}\text { Direct } \\
\text { Crossover }\end{array}$ & $\begin{array}{l}\text { Work } \rightarrow \\
\text { Home }\end{array}$ & $\begin{array}{l}\text { Job stress to } \\
\text { home stress }\end{array}$ & $\begin{array}{l}\text { Bidirectional } \\
\text { (Spouse to } \\
\text { spouse) }\end{array}$ & $\begin{array}{l}\text { One spouses' job stress } \\
\text { affected the other spouses' } \\
\text { stress at home }\end{array}$ \\
\hline Booth (1977) & 560 couples & $\begin{array}{l}\text { Stressor } \rightarrow \\
\text { Strain }\end{array}$ & $\begin{array}{l}\text { Direct } \\
\text { Crossover }\end{array}$ & $\begin{array}{l}\text { Work } \rightarrow \\
\text { Home }\end{array}$ & $\begin{array}{l}\text { Employment } \\
\text { status on } \\
\text { marital } \\
\text { satisfaction }\end{array}$ & $\begin{array}{l}\text { Unidirectional } \\
\text { (Wife to } \\
\text { husband) }\end{array}$ & $\begin{array}{l}\text { Wife's employment had no } \\
\text { affect on husband's marital } \\
\text { satisfaction }\end{array}$ \\
\hline $\begin{array}{l}\text { Brockwood, } \\
\text { Hammer, Neal }\end{array}$ & $\begin{array}{l}618 \\
\text { individuals }\end{array}$ & $\begin{array}{l}\text { Stressor } \rightarrow \\
\text { Strain }\end{array}$ & $\begin{array}{l}\text { Direct } \\
\text { Crossover }\end{array}$ & $\begin{array}{l}\text { Home } \rightarrow \\
\text { Home }\end{array}$ & $\begin{array}{l}\text { Work and } \\
\text { family }\end{array}$ & $\begin{array}{l}\text { Bidirectional } \\
\text { (Spouse to }\end{array}$ & $\begin{array}{l}\text { Extent to which one's } \\
\text { spouse made }\end{array}$ \\
\hline
\end{tabular}




\begin{tabular}{|c|c|c|c|c|c|c|c|}
\hline Citation & Sample & $\begin{array}{l}\text { Type of } \\
\text { Crossover }\end{array}$ & Mechanism & $\begin{array}{l}\text { Origin/ } \\
\text { Destination }\end{array}$ & Crossover of & Directionality & Findings \\
\hline $\begin{array}{l}\text { \& Colton } \\
\text { (2001) }\end{array}$ & $\begin{array}{l}\text { comprising } \\
\text { dual-earner } \\
\text { couples }\end{array}$ & & & & $\begin{array}{l}\text { accommoda- } \\
\text { tions to work } \\
\text { and family } \\
\text { attitudes }\end{array}$ & spouse) & $\begin{array}{l}\text { accommodations at home } \\
\text { did have a significant } \\
\text { negative impact on one's } \\
\text { own level of family } \\
\text { satisfaction for both } \\
\text { husbands and wives }\end{array}$ \\
\hline $\begin{array}{l}\text { Burke, Weir \& } \\
\text { Duwors } \\
\text { (1980) }\end{array}$ & $\begin{array}{l}85 \text { Senior } \\
\text { administrators } \\
\text { of correctional } \\
\text { institutes and } \\
\text { spouses }\end{array}$ & $\begin{array}{l}\text { Stressor } \rightarrow \\
\text { Strain }\end{array}$ & $\begin{array}{l}\text { Direct } \\
\text { Crossover }\end{array}$ & $\begin{array}{l}\text { Work } \rightarrow \\
\text { Home }\end{array}$ & $\begin{array}{l}\text { Job demands } \\
\text { on marital/life } \\
\text { satisfaction, } \\
\text { social } \\
\text { participation } \\
\text { and well-being }\end{array}$ & $\begin{array}{l}\text { Unidirectional } \\
\text { (Husband to } \\
\text { wife) }\end{array}$ & $\begin{array}{l}\text { Husbands' job demands } \\
\text { were related to less marital } \\
\text { and life satisfaction, } \\
\text { decreased social } \\
\text { participation, and increased } \\
\text { psychosomatic symptoms } \\
\text { and negative feeling states o } \\
\text { wives }\end{array}$ \\
\hline $\begin{array}{l}\text { Chan \& } \\
\text { Margolin } \\
\text { (1994) }\end{array}$ & $\begin{array}{l}59 \text { dual-earner } \\
\text { couples }\end{array}$ & $\begin{array}{l}\text { Strain } \rightarrow \\
\text { Strain }\end{array}$ & $\begin{array}{l}\text { Direct } \\
\text { Crossover }\end{array}$ & $\begin{array}{l}\text { Work } \rightarrow \\
\text { Home; } \\
\text { Home } \rightarrow \\
\text { Work }\end{array}$ & $\begin{array}{l}\text { Work mood } \\
\text { and home } \\
\text { affect }\end{array}$ & $\begin{array}{l}\text { Unidirectional } \\
\text { (Wife to } \\
\text { husband) }\end{array}$ & $\begin{array}{l}\text { Wives' negative work mood } \\
\text { and work fatigue crossed } \\
\text { over to husbands' reactions } \\
\text { at home; wives' home affect } \\
\text { had a significant relationshi } \\
\text { with husbands' work mood }\end{array}$ \\
\hline $\begin{array}{l}\text { Cinamon, } \\
\text { Weisel \& } \\
\text { Tzuk (2007) }\end{array}$ & $\begin{array}{l}60 \text { Israeli } \\
\text { married } \\
\text { couples }\end{array}$ & $\begin{array}{l}\text { Stressor } \rightarrow \\
\text { Stressor }\end{array}$ & $\begin{array}{l}\text { Direct } \\
\text { Crossover }\end{array}$ & $\begin{array}{l}\text { Work } \rightarrow \\
\text { Home }\end{array}$ & $\begin{array}{l}\text { Work-to- } \\
\text { family conflict } \\
\text { and family-to- } \\
\text { work conflict }\end{array}$ & $\begin{array}{l}\text { Bidirectional } \\
\text { (Spouse to } \\
\text { spouse) }\end{array}$ & $\begin{array}{l}\text { Work-to-family conflict of } \\
\text { one spouse was positively } \\
\text { correlated with family-to- } \\
\text { work conflict for the other } \\
\text { spouse }\end{array}$ \\
\hline Cronkite \& & 267 married & Strain $\rightarrow$ & Direct & Home $\rightarrow$ & Stress and & Bidirectional & Respondents who report \\
\hline
\end{tabular}




\begin{tabular}{|c|c|c|c|c|c|c|c|}
\hline Citation & Sample & $\begin{array}{l}\text { Type of } \\
\text { Crossover }\end{array}$ & Mechanism & $\begin{array}{l}\text { Origin/ } \\
\text { Destination }\end{array}$ & Crossover of & Directionality & Findings \\
\hline Moos (1984) & couples & Strain & Crossover & Home & distress & $\begin{array}{l}\text { (Spouse to } \\
\text { spouse) }\end{array}$ & $\begin{array}{l}\text { more depressive symptoms } \\
\text { are likely to experience the } \\
\text { ongoing stress of similar } \\
\text { depressive symptoms } \\
\text { among their spouses }\end{array}$ \\
\hline $\begin{array}{l}\text { Crossfield, } \\
\text { Kinman \& } \\
\text { Jones (2005) }\end{array}$ & $\begin{array}{l}74 \text { dual-earner } \\
\text { couples }\end{array}$ & $\begin{array}{l}\text { Stressor } \rightarrow \\
\text { Strain }\end{array}$ & $\begin{array}{l}\text { Direct } \\
\text { Crossover }\end{array}$ & $\begin{array}{l}\text { Work } \rightarrow \\
\text { Home }\end{array}$ & $\begin{array}{l}\text { Stressors to } \\
\text { anxiety and } \\
\text { depression }\end{array}$ & $\begin{array}{l}\text { Unidirectional } \\
\text { (Wife to } \\
\text { husband) }\end{array}$ & $\begin{array}{l}\text { Positive relationships were } \\
\text { found between women's } \\
\text { work stressors and the } \\
\text { anxiety and depression } \\
\text { reported by their male } \\
\text { partners }\end{array}$ \\
\hline $\begin{array}{l}\text { Demerouti, } \\
\text { Bakker \& } \\
\text { Schaufeli } \\
(2005)\end{array}$ & $\begin{array}{l}191 \text { dual- } \\
\text { earner parents } \\
\text { (in couples) }\end{array}$ & $\begin{array}{l}\text { Strain } \rightarrow \\
\text { Strain; } \\
\text { Resource } \rightarrow \\
\text { Resource }\end{array}$ & $\begin{array}{l}\text { Direct } \\
\text { Crossover }\end{array}$ & $\begin{array}{l}\text { Home } \rightarrow \\
\text { Home }\end{array}$ & $\begin{array}{l}\text { Job exhaustion } \\
\text { and life } \\
\text { satisfaction }\end{array}$ & $\begin{array}{l}\text { Unidirectional } \\
\text { (Female to } \\
\text { male; male to } \\
\text { female) }\end{array}$ & $\begin{array}{l}\text { Found a crossover path from } \\
\text { females' exhaustion to } \\
\text { males' exhaustion and from } \\
\text { males' life satisfaction to } \\
\text { females' life satisfaction }\end{array}$ \\
\hline $\begin{array}{l}\text { Dikkers, } \\
\text { Geurts, } \\
\text { Kinnunen, } \\
\text { Kompier \& } \\
\text { Taris (2007) }\end{array}$ & 253 couples & $\begin{array}{l}\text { Stressor } \rightarrow \\
\text { Stressor; } \\
\text { Strain } \rightarrow \\
\text { Stressor; } \\
\text { Strain } \rightarrow \\
\text { Strain }\end{array}$ & $\begin{array}{l}\text { Direct } \\
\text { Crossover }\end{array}$ & $\begin{array}{l}\text { Work } \rightarrow \\
\text { Home; Home } \\
\rightarrow \text { Home; } \\
\text { Home } \rightarrow \\
\text { Home }\end{array}$ & $\begin{array}{l}\text { Work load, } \\
\text { psychological } \\
\text { health, home } \\
\text { load }\end{array}$ & $\begin{array}{l}\text { Unidirectional } \\
\text { (Husband to } \\
\text { wife); and } \\
\text { Bidirectional } \\
\text { (Spouse to } \\
\text { spouse) }\end{array}$ & $\begin{array}{l}\text { When husbands reported } \\
\text { higher work load and more } \\
\text { psychological health } \\
\text { complaints, their wives } \\
\text { experienced higher home } \\
\text { load; wives' and husbands' } \\
\text { psychological health were } \\
\text { associated }\end{array}$ \\
\hline $\begin{array}{l}\text { Eckenrode \& } \\
\text { Gore (1981) }\end{array}$ & 356 women & $\begin{array}{l}\text { Stressor } \rightarrow \\
\text { Strain }\end{array}$ & $\begin{array}{l}\text { Direct } \\
\text { Crossover }\end{array}$ & $\begin{array}{l}\text { Home } \rightarrow \\
\text { Home }\end{array}$ & $\begin{array}{l}\text { Life events to } \\
\text { health }\end{array}$ & $\begin{array}{l}\text { Unidirectional } \\
\text { (Male to }\end{array}$ & $\begin{array}{l}\text { Frequency of significant } \\
\text { others' life events affected }\end{array}$ \\
\hline
\end{tabular}




\begin{tabular}{|c|c|c|c|c|c|c|c|}
\hline Citation & Sample & $\begin{array}{l}\text { Type of } \\
\text { Crossover }\end{array}$ & Mechanism & $\begin{array}{l}\text { Origin/ } \\
\text { Destination }\end{array}$ & Crossover of & Directionality & Findings \\
\hline & & & & & & female) & $\begin{array}{l}\text { women's health status and } \\
\text { health behavior }\end{array}$ \\
\hline $\begin{array}{l}\text { Etzion \& } \\
\text { Westman } \\
(2001)\end{array}$ & 57 couples & $\begin{array}{l}\text { Strain } \rightarrow \\
\text { Strain }\end{array}$ & $\begin{array}{l}\text { Direct } \\
\text { Crossover }\end{array}$ & $\begin{array}{l}\text { Home } \rightarrow \\
\text { Home }\end{array}$ & Strain & $\begin{array}{l}\text { Bidirectional } \\
\text { (Partner to } \\
\text { partner) }\end{array}$ & $\begin{array}{l}\text { Both studies show that more } \\
\text { strain crossed over from one } \\
\text { partner to the other under } \\
\text { stressful conditions (i.e., } \\
\text { before vacation or exams), } \\
\text { than during the more relaxec } \\
\text { period (i.e., immediately } \\
\text { after vacation, at the } \\
\text { beginning of an MBA } \\
\text { program) }\end{array}$ \\
\hline $\begin{array}{l}\text { Fletcher } \\
(1983)\end{array}$ & $\begin{array}{l}\text { Information } \\
\text { on } 1,088,995 \\
\text { cases of death }\end{array}$ & $\begin{array}{l}\text { Stressor } \rightarrow \\
\text { Strain }\end{array}$ & $\begin{array}{l}\text { Direct } \\
\text { Crossover }\end{array}$ & $\begin{array}{l}\text { Work } \rightarrow \\
\text { Home }\end{array}$ & $\begin{array}{l}\text { Male } \\
\text { occupational } \\
\text { mortality on } \\
\text { female's } \\
\text { mortality }\end{array}$ & $\begin{array}{l}\text { Unidirectional } \\
\text { (Male to } \\
\text { female) }\end{array}$ & $\begin{array}{l}\text { Job risks of the husband } \\
\text { affect the life expectancy of } \\
\text { the husband and the wife }\end{array}$ \\
\hline $\begin{array}{l}\text { Fletcher } \\
\text { (1988) }\end{array}$ & $\begin{array}{l}324,822 \\
\text { British men in } \\
556 \\
\text { occupations } \\
\text { and } 35,915 \\
\text { wives }\end{array}$ & $\begin{array}{l}\text { Stressor } \rightarrow \\
\text { Strain }\end{array}$ & $\begin{array}{l}\text { Direct } \\
\text { Crossover }\end{array}$ & $\begin{array}{l}\text { Work } \rightarrow \\
\text { Home }\end{array}$ & $\begin{array}{l}\text { Male } \\
\text { occupational } \\
\text { mortality on } \\
\text { female's } \\
\text { mortality and } \\
\text { cause of death }\end{array}$ & $\begin{array}{l}\text { Unidirectional } \\
\text { (Male to } \\
\text { female) }\end{array}$ & $\begin{array}{l}\text { Occupational mortality risk } \\
\text { of husbands affects wives' } \\
\text { life expectancy and cause of } \\
\text { death }\end{array}$ \\
\hline $\begin{array}{l}\text { Gareis, } \\
\text { Barnett \& } \\
\text { Brennan }\end{array}$ & $\begin{array}{l}105 \text { female } \\
\text { reduced hour } \\
\text { physicians and }\end{array}$ & $\begin{array}{l}\text { Stressor } \rightarrow \\
\text { Strain }\end{array}$ & $\begin{array}{l}\text { Direct } \\
\text { Crossover }\end{array}$ & $\begin{array}{l}\text { Work } \rightarrow \\
\text { Home }\end{array}$ & $\begin{array}{l}\text { Work } \\
\text { schedule fit on } \\
\text { marital role }\end{array}$ & $\begin{array}{l}\text { Unidirectional } \\
\text { (Husband to } \\
\text { wife; wife to }\end{array}$ & $\begin{array}{l}\text { Husband's ratings of own } \\
\text { schedule-fit predicted wives } \\
\text { marital role quality; wives' }\end{array}$ \\
\hline
\end{tabular}




\begin{tabular}{|c|c|c|c|c|c|c|c|}
\hline Citation & Sample & $\begin{array}{l}\text { Type of } \\
\text { Crossover }\end{array}$ & Mechanism & $\begin{array}{l}\text { Origin/ } \\
\text { Destination }\end{array}$ & Crossover of & Directionality & Findings \\
\hline (2003) & $\begin{array}{l}\text { their full-time } \\
\text { employed } \\
\text { husbands }\end{array}$ & & & & $\begin{array}{l}\text { quality and } \\
\text { psychological } \\
\text { distress }\end{array}$ & husband) & $\begin{array}{l}\text { ratings of own schedule fit } \\
\text { predicted husbands } \\
\text { psychological distress. }\end{array}$ \\
\hline $\begin{array}{l}\text { Gorgievski- } \\
\text { Duijvesteijn, } \\
\text { Giesen \& } \\
\text { Bakker (2000) }\end{array}$ & $\begin{array}{l}91 \text { Dutch } \\
\text { dairy farm } \\
\text { couples }\end{array}$ & $\begin{array}{l}\text { Strain } \rightarrow \\
\text { Stressor; } \\
\text { Strain } \rightarrow \\
\text { Strain }\end{array}$ & $\begin{array}{l}\text { Direct } \\
\text { Crossover }\end{array}$ & $\begin{array}{l}\text { Home } \rightarrow \\
\text { Home }\end{array}$ & $\begin{array}{l}\text { Financial } \\
\text { problems to } \\
\text { health; health } \\
\text { to health }\end{array}$ & $\begin{array}{l}\text { Unidirectional } \\
\text { (Husband to } \\
\text { wife) }\end{array}$ & $\begin{array}{l}\text { Financial problems were not } \\
\text { predictive of health } \\
\text { complaints; husband's health } \\
\text { complaints predicted the } \\
\text { couples financial problems } \\
\text { and wives' health complaints } \\
10 \text { years later }\end{array}$ \\
\hline $\begin{array}{l}\text { Green, et al. } \\
(2010)\end{array}$ & $\begin{array}{l}139 \text { faculty } \\
\text { members and } \\
\text { their partners }\end{array}$ & $\begin{array}{l}\text { Strain } \rightarrow \\
\text { Strain }\end{array}$ & $\begin{array}{l}\text { Indirect } \\
\text { Crossover }\end{array}$ & $\begin{array}{l}\text { Home } \rightarrow \\
\text { Work }\end{array}$ & $\begin{array}{l}\text { Negative } \\
\text { emotional } \\
\text { displays on } \\
\text { work-related } \\
\text { attitudes }\end{array}$ & $\begin{array}{l}\text { Unidirectional } \\
\text { (Partner to } \\
\text { Faculty } \\
\text { member) }\end{array}$ & $\begin{array}{l}\text { Negative emotional displays } \\
\text { were found to be } \\
\text { significantly } \\
\text { and positively related to } \\
\text { focal employee turnover } \\
\text { exploration activities and } \\
\text { negatively } \\
\text { related to focal employee } \\
\text { career resilience }\end{array}$ \\
\hline $\begin{array}{l}\text { Greenhaus \& } \\
\text { Parasuraman } \\
(1987)\end{array}$ & 425 couples & $\begin{array}{l}\text { Resource } \rightarrow \\
\text { Resource }\end{array}$ & $\begin{array}{l}\text { Direct } \\
\text { Crossover }\end{array}$ & $\begin{array}{l}\text { Work } \rightarrow \\
\text { Work; Work } \\
\rightarrow \text { Home }\end{array}$ & $\begin{array}{l}\text { Employment } \\
\text { status to job } \\
\text { satisfaction } \\
\text { and quality of } \\
\text { life }\end{array}$ & $\begin{array}{l}\text { Unidirectional } \\
\text { (Wife to } \\
\text { husband) }\end{array}$ & $\begin{array}{l}\text { Wife's employment was } \\
\text { positively related to } \\
\text { husband's job satisfaction } \\
\text { and quality of life }\end{array}$ \\
\hline $\begin{array}{l}\text { Haines, } \\
\text { Marchand \& }\end{array}$ & $\begin{array}{l}2,904 \text { dual- } \\
\text { earner couples }\end{array}$ & $\begin{array}{l}\text { Stressor } \rightarrow \\
\text { Strain }\end{array}$ & $\begin{array}{l}\text { Direct } \\
\text { Crossover; }\end{array}$ & $\begin{array}{l}\text { Work } \rightarrow \\
\text { Home }\end{array}$ & $\begin{array}{l}\text { Stress } \\
\text { resulting from }\end{array}$ & $\begin{array}{l}\text { Bidirectional } \\
\text { (Spouse to }\end{array}$ & $\begin{array}{l}\text { Workplace aggression was } \\
\text { related to higher levels of }\end{array}$ \\
\hline
\end{tabular}




\begin{tabular}{|c|c|c|c|c|c|c|c|}
\hline Citation & Sample & $\begin{array}{l}\text { Type of } \\
\text { Crossover }\end{array}$ & Mechanism & $\begin{array}{l}\text { Origin/ } \\
\text { Destination }\end{array}$ & Crossover of & Directionality & Findings \\
\hline Harvey (2006) & & & $\begin{array}{l}\text { Common } \\
\text { Stressors }\end{array}$ & & $\begin{array}{l}\text { workplace } \\
\text { aggression }\end{array}$ & spouse) & $\begin{array}{l}\text { psychological distress in } \\
\text { spouse; controlled for other } \\
\text { possible stressors (decision } \\
\text { authority on the job, working } \\
\text { hours, irregular shifts, } \\
\text { marital strain), age and } \\
\text { gender. }\end{array}$ \\
\hline $\begin{array}{l}\text { Hammer, } \\
\text { Allen \& } \\
\text { Grigsby } \\
\text { (1997) }\end{array}$ & $\begin{array}{l}399 \text { dual- } \\
\text { earner couples }\end{array}$ & $\begin{array}{l}\text { Stressor } \rightarrow \\
\text { Stressor }\end{array}$ & $\begin{array}{l}\text { Direct } \\
\text { Crossover }\end{array}$ & $\begin{array}{l}\text { Work } \rightarrow \\
\text { Home }\end{array}$ & $\begin{array}{l}\text { Work-family } \\
\text { conflict }\end{array}$ & $\begin{array}{l}\text { Bidirectional } \\
\text { (Spouse to } \\
\text { spouse) }\end{array}$ & $\begin{array}{l}\text { Individual's level of work- } \\
\text { family conflict was a } \\
\text { significant predictor of his or } \\
\text { her partner's level of work- } \\
\text { family conflict }\end{array}$ \\
\hline $\begin{array}{l}\text { Hammer, } \\
\text { Bauer \& } \\
\text { Grandey } \\
(2003)\end{array}$ & $\begin{array}{l}359 \text { dual- } \\
\text { earner couples }\end{array}$ & $\begin{array}{l}\text { Stressor } \rightarrow \\
\text { Strain }\end{array}$ & $\begin{array}{l}\text { Direct } \\
\text { Crossover }\end{array}$ & $\begin{array}{l}\text { Home } \rightarrow \\
\text { Work; Work } \\
\rightarrow \text { Home }\end{array}$ & $\begin{array}{l}\text { Work-to- } \\
\text { family and } \\
\text { family-to- } \\
\text { work conflict } \\
\text { on withdrawal } \\
\text { behaviors }\end{array}$ & $\begin{array}{l}\text { Unidirectional } \\
\text { (Wife to } \\
\text { husband; } \\
\text { husband to } \\
\text { wife) }\end{array}$ & $\begin{array}{l}\text { Husband interruptions were } \\
\text { predicted by wife family-to- } \\
\text { work conflict; wife lateness } \\
\text { was predicted by husband } \\
\text { family-to-work conflict }\end{array}$ \\
\hline $\begin{array}{l}\text { Hammer, } \\
\text { Cullen, Neal, } \\
\text { Sinclair \& } \\
\text { Shafiro (2005) }\end{array}$ & $\begin{array}{l}234 \text { dual- } \\
\text { earner couples }\end{array}$ & $\begin{array}{l}\text { Resource } \rightarrow \\
\text { Strain; } \\
\text { Stressor } \rightarrow \\
\text { Strain }\end{array}$ & $\begin{array}{l}\text { Direct } \\
\text { Crossover }\end{array}$ & $\begin{array}{l}\text { Work } \rightarrow \\
\text { Home }\end{array}$ & $\begin{array}{l}\text { Positive } \\
\text { spillover; } \\
\text { work-family } \\
\text { conflict }\end{array}$ & $\begin{array}{l}\text { Unidirectional } \\
\text { (Wife to } \\
\text { husband; } \\
\text { husband to } \\
\text { wife) }\end{array}$ & $\begin{array}{l}\text { Husbands' work-to-family } \\
\text { positive spillover was related } \\
\text { to reduced depression for } \\
\text { wives over time; wives' } \\
\text { family-to-work positive } \\
\text { spillover was related to } \\
\text { reduced depression for } \\
\text { husbands over time; } \\
\text { husbands' work-family }\end{array}$ \\
\hline
\end{tabular}




\begin{tabular}{|c|c|c|c|c|c|c|c|}
\hline Citation & Sample & $\begin{array}{l}\text { Type of } \\
\text { Crossover }\end{array}$ & Mechanism & $\begin{array}{l}\text { Origin/ } \\
\text { Destination }\end{array}$ & Crossover of & Directionality & Findings \\
\hline & & & & & & & $\begin{array}{l}\text { conflict at time } 2 \text { was } \\
\text { significantly related to } \\
\text { wives' depression at time } 2\end{array}$ \\
\hline $\begin{array}{l}\text { Hammer, } \\
\text { Neal, } \\
\text { Newsom, } \\
\text { Brockwood \& } \\
\text { Colton (2005) }\end{array}$ & $\begin{array}{l}234 \text { dual- } \\
\text { earner couples }\end{array}$ & $\begin{array}{l}\text { Resource } \rightarrow \\
\text { Stressor; } \\
\text { Resource } \\
\rightarrow \text { Resource }\end{array}$ & $\begin{array}{l}\text { Direct } \\
\text { Crossover }\end{array}$ & $\begin{array}{l}\text { Work } \rightarrow \\
\text { Home; Work } \\
\rightarrow \text { Home }\end{array}$ & $\begin{array}{l}\text { Use of } \\
\text { dependent } \\
\text { care supports } \\
\text { and work- } \\
\text { family } \\
\text { conflict; use } \\
\text { of alternative } \\
\text { work } \\
\text { arrangements } \\
\text { and job } \\
\text { satisfaction }\end{array}$ & $\begin{array}{l}\text { Unidirectional } \\
\text { (Couple to } \\
\text { wife) }\end{array}$ & $\begin{array}{l}\text { Use of dependent care } \\
\text { supports by the couple was } \\
\text { associated with high levels } \\
\text { of work-family conflict } \\
\text { (both directions) of the wife } \\
\text { Significant positive } \\
\text { relationship between use of } \\
\text { alternative work } \\
\text { arrangements by the couple } \\
\text { and wives' job satisfaction }\end{array}$ \\
\hline $\begin{array}{l}\text { Haynes, Eaker } \\
\text { \& Feinleib } \\
\text { (1983) }\end{array}$ & 269 couples & $\begin{array}{l}\text { Stressor } \rightarrow \\
\text { Strain }\end{array}$ & $\begin{array}{l}\text { Direct } \\
\text { Crossover }\end{array}$ & $\begin{array}{l}\text { Work } \rightarrow \\
\text { Home }\end{array}$ & $\begin{array}{l}\text { Employment } \\
\text { on CHD }\end{array}$ & $\begin{array}{l}\text { Unidirectional } \\
\text { (Wife to } \\
\text { husband) }\end{array}$ & $\begin{array}{l}\text { Husbands of wives in white- } \\
\text { collar jobs were over } 3 \text { time } \\
\text { more likely to develop CHD } \\
\text { than those married to blue- } \\
\text { collar workers or } \\
\text { housewives }\end{array}$ \\
\hline $\begin{array}{l}\text { Howe, Levy \& } \\
\text { Caplan (2004) }\end{array}$ & $\begin{array}{l}252 \text { couples } \\
\text { (including one } \\
\text { unemployed) }\end{array}$ & $\begin{array}{l}\text { Stressor } \rightarrow \\
\text { Strain }\end{array}$ & $\begin{array}{l}\text { Direct } \\
\text { Crossover; } \\
\text { Indirect } \\
\text { Crossover; } \\
\text { Common } \\
\text { Stressors }\end{array}$ & $\begin{array}{l}\text { Work } \rightarrow \\
\text { Home }\end{array}$ & $\begin{array}{l}\text { Stress from } \\
\text { job loss, } \\
\text { depression }\end{array}$ & $\begin{array}{l}\text { Unidirectional } \\
\text { (Job seeker to } \\
\text { spouse) }\end{array}$ & $\begin{array}{l}\text { Secondary stressors after jol } \\
\text { loss are associated with } \\
\text { increases in depressive } \\
\text { symptoms for the job seeker } \\
\text { and the partner. Emotional } \\
\text { reactions in the two } \\
\text { members of the couple are }\end{array}$ \\
\hline
\end{tabular}




\begin{tabular}{|c|c|c|c|c|c|c|c|}
\hline Citation & Sample & $\begin{array}{l}\text { Type of } \\
\text { Crossover }\end{array}$ & Mechanism & $\begin{array}{l}\text { Origin/ } \\
\text { Destination }\end{array}$ & Crossover of & Directionality & Findings \\
\hline & & & & & & & $\begin{array}{l}\text { not independent and may be } \\
\text { mutually reinforcing. } \\
\text { Secondary stressors } \\
\text { degrade the quality of the } \\
\text { couples' relationship, and } \\
\text { this in turn contributes to } \\
\text { increased distress. }\end{array}$ \\
\hline $\begin{array}{l}\text { Jackson \& } \\
\text { Maslach } \\
(1982)\end{array}$ & $\begin{array}{l}142 \text { police } \\
\text { officers and } \\
\text { spouses }\end{array}$ & $\begin{array}{l}\text { Stressor } \rightarrow \\
\text { Strain }\end{array}$ & $\begin{array}{l}\text { Direct } \\
\text { Crossover }\end{array}$ & $\begin{array}{l}\text { Work } \rightarrow \\
\text { Home }\end{array}$ & $\begin{array}{l}\text { Job stress on } \\
\text { dissatisfaction } \\
\text { and distress }\end{array}$ & $\begin{array}{l}\text { Unidirectional } \\
\text { (Husband to } \\
\text { wife) }\end{array}$ & $\begin{array}{l}\text { Husbands' job stress } \\
\text { affected the dissatisfaction } \\
\text { and distress of the wife }\end{array}$ \\
\hline $\begin{array}{l}\text { Jackson, } \\
\text { Zebeck \& } \\
\text { Summers } \\
(1985)\end{array}$ & $\begin{array}{l}95 \text { plant } \\
\text { operators and } \\
\text { their spouses }\end{array}$ & $\begin{array}{l}\text { Stressor } \rightarrow \\
\text { Strain }\end{array}$ & $\begin{array}{l}\text { Direct } \\
\text { Crossover }\end{array}$ & $\begin{array}{l}\text { Work } \rightarrow \\
\text { Home }\end{array}$ & $\begin{array}{l}\text { Job } \\
\text { experiences to } \\
\text { quality of life } \\
\text { and } \\
\text { dissatisfaction } \\
\text { with spouse's } \\
\text { job }\end{array}$ & $\begin{array}{l}\text { Unidirectional } \\
\text { (Husband to } \\
\text { wife) }\end{array}$ & $\begin{array}{l}\text { Emotional interference } \\
\text { caused by the husbands' job } \\
\text { experiences was related to } \\
\text { wives' quality of life and } \\
\text { dissatisfaction with the } \\
\text { husbands' job }\end{array}$ \\
\hline $\begin{array}{l}\text { Jones \& } \\
\text { Fletcher } \\
(1993 a)\end{array}$ & $\begin{array}{l}60 \text { working } \\
\text { couples }\end{array}$ & $\begin{array}{l}\text { Stressor } \rightarrow \\
\text { Strain }\end{array}$ & $\begin{array}{l}\text { Direct } \\
\text { Crossover }\end{array}$ & $\begin{array}{l}\text { Work } \rightarrow \\
\text { Home }\end{array}$ & $\begin{array}{l}\text { Work } \\
\text { demands to } \\
\text { psychological } \\
\text { health }\end{array}$ & $\begin{array}{l}\text { Unidirectional } \\
\text { (Male to } \\
\text { female) }\end{array}$ & $\begin{array}{l}\text { Transmission of stress from } \\
\text { men to women, especially } \\
\text { when men work in high } \\
\text { strain jobs, but no } \\
\text { corresponding transmission } \\
\text { from women to men; } \\
\text { Husbands' interpersonal } \\
\text { work situation correlated } \\
\text { with wives' anxiety and } \\
\text { depression, and husbands' }\end{array}$ \\
\hline
\end{tabular}




\begin{tabular}{|c|c|c|c|c|c|c|c|}
\hline Citation & Sample & $\begin{array}{l}\text { Type of } \\
\text { Crossover }\end{array}$ & Mechanism & $\begin{array}{l}\text { Origin/ } \\
\text { Destination }\end{array}$ & Crossover of & Directionality & Findings \\
\hline & & & & & & & $\begin{array}{l}\text { supports with wives' } \\
\text { depression }\end{array}$ \\
\hline $\begin{array}{l}\text { Jones \& } \\
\text { Fletcher } \\
(1993 b)\end{array}$ & $\begin{array}{l}60 \text { working } \\
\text { couples }\end{array}$ & $\begin{array}{l}\text { Stressor } \rightarrow \\
\text { Strain }\end{array}$ & $\begin{array}{l}\text { Direct } \\
\text { Crossover }\end{array}$ & $\begin{array}{l}\text { Work } \rightarrow \\
\text { Home }\end{array}$ & $\begin{array}{l}\text { Work stress to } \\
\text { psychological } \\
\text { and physical } \\
\text { health }\end{array}$ & $\begin{array}{l}\text { Bidirectional } \\
\text { (Spouse to } \\
\text { spouse) }\end{array}$ & $\begin{array}{l}\text { Daily fluctuations in work } \\
\text { stressors affect both spouses }\end{array}$ \\
\hline $\begin{array}{l}\text { Jones \& } \\
\text { Fletcher } \\
(1996)\end{array}$ & 20 couples & $\begin{array}{l}\text { Stressor } \rightarrow \\
\text { Strain }\end{array}$ & $\begin{array}{l}\text { Direct } \\
\text { Crossover }\end{array}$ & $\begin{array}{l}\text { Home } \rightarrow \\
\text { Home }\end{array}$ & $\begin{array}{l}\text { Domestic } \\
\text { stressors }\end{array}$ & $\begin{array}{l}\text { Unidirectional } \\
\text { (Wife to } \\
\text { husband) }\end{array}$ & $\begin{array}{l}\text { Wives' domestic stressors } \\
\text { were correlated with } \\
\text { husbands' sleep for the } \\
\text { subsequent night; did not } \\
\text { show any direct relationship } \\
\text { between individual's work } \\
\text { stressors and partner's } \\
\text { moods }\end{array}$ \\
\hline $\begin{array}{l}\text { Katz, Beach \& } \\
\text { Joiner (1999) }\end{array}$ & $\begin{array}{l}\text { Undergraduate } \\
\text { dating couples } \\
(\mathrm{N}=105)\end{array}$ & $\begin{array}{l}\text { Strain } \rightarrow \\
\text { Strain }\end{array}$ & $\begin{array}{l}\text { Direct } \\
\text { Crossover }\end{array}$ & $\begin{array}{l}\text { Home } \rightarrow \\
\text { Home }\end{array}$ & $\begin{array}{l}\text { Depressive } \\
\text { symptoms }\end{array}$ & $\begin{array}{l}\text { Bidirectional } \\
\text { (Dating } \\
\text { partner to } \\
\text { dating partner) }\end{array}$ & $\begin{array}{l}\text { Partners' levels of } \\
\text { depressive symptoms were } \\
\text { related (even after } \\
\text { controlling for relationship } \\
\text { satisfaction) }\end{array}$ \\
\hline $\begin{array}{l}\text { Katz, } \\
\text { Monnier, } \\
\text { Libet, Shaw \& } \\
\text { Beach (2000) }\end{array}$ & $\begin{array}{l}30 \text { medical } \\
\text { student } \\
\text { marriages }\end{array}$ & $\begin{array}{l}\text { Stressor } \rightarrow \\
\text { Strain }\end{array}$ & $\begin{array}{l}\text { Direct } \\
\text { Crossover }\end{array}$ & $\begin{array}{l}\text { Work } \rightarrow \\
\text { Home }\end{array}$ & $\begin{array}{l}\text { Stress on } \\
\text { emotional } \\
\text { adjustment }\end{array}$ & $\begin{array}{l}\text { Unidirectional } \\
\text { (Medical } \\
\text { student to } \\
\text { spouse) }\end{array}$ & $\begin{array}{l}\text { Medical students' perceived } \\
\text { stress was significantly } \\
\text { associated with their } \\
\text { spouses' emotional } \\
\text { adjustment }\end{array}$ \\
\hline Kossek, & 51 mother- & Resource $\rightarrow$ & Direct & Home $\rightarrow$ & Higher quality & Unidirectional & Mothers using care from \\
\hline
\end{tabular}




\begin{tabular}{|c|c|c|c|c|c|c|c|}
\hline Citation & Sample & $\begin{array}{l}\text { Type of } \\
\text { Crossover }\end{array}$ & Mechanism & $\begin{array}{l}\text { Origin/ } \\
\text { Destination }\end{array}$ & Crossover of & Directionality & Findings \\
\hline $\begin{array}{l}\text { Pichler, } \\
\text { Meece \& } \\
\text { Barratt (2008) }\end{array}$ & provider pairs & $\begin{array}{l}\text { Stressor; } \\
\text { Resource } \rightarrow \\
\text { Strain }\end{array}$ & Crossover & Home & $\begin{array}{l}\text { care intentions } \\
\text { to work- } \\
\text { family conflict } \\
\text { and depression }\end{array}$ & $\begin{array}{l}\text { (Child-care } \\
\text { provider to } \\
\text { mother) }\end{array}$ & $\begin{array}{l}\text { providers who reported } \\
\text { higher quality parent- } \\
\text { caregiver social relationships } \\
\text { reported lower work-family } \\
\text { conflict and depressive } \\
\text { symptoms }\end{array}$ \\
\hline $\begin{array}{l}\text { Lavee \& Ben- } \\
\text { Ari }(2007)\end{array}$ & $\begin{array}{l}169 \text { Israeli } \\
\text { dual-earner } \\
\text { couples }\end{array}$ & $\begin{array}{l}\text { Stressor } \rightarrow \\
\text { Strain }\end{array}$ & $\begin{array}{l}\text { Direct } \\
\text { Crossover }\end{array}$ & $\begin{array}{l}\text { Work } \rightarrow \\
\text { Home }\end{array}$ & $\begin{array}{l}\text { Work stress to } \\
\text { partner's } \\
\text { mood at home }\end{array}$ & $\begin{array}{l}\text { Bidirectional } \\
\text { (Spouse to } \\
\text { spouse) }\end{array}$ & $\begin{array}{l}\text { Crossover effect from one's } \\
\text { work stress to partners' } \\
\text { mood is moderated by the } \\
\text { couples' global evaluation of } \\
\text { marital quality (higher) }\end{array}$ \\
\hline $\begin{array}{l}\text { Long \& Voges } \\
\text { (1987) }\end{array}$ & $\begin{array}{l}301 \text { prison } \\
\text { officers and } \\
\text { their wives }\end{array}$ & $\begin{array}{l}\text { Stressor } \rightarrow \\
\text { Strain }\end{array}$ & $\begin{array}{l}\text { Direct } \\
\text { Crossover }\end{array}$ & $\begin{array}{l}\text { Work } \rightarrow \\
\text { Home }\end{array}$ & $\begin{array}{l}\text { Job stress to } \\
\text { psychological } \\
\text { well-being }\end{array}$ & $\begin{array}{l}\text { Unidirectional } \\
\text { (Husband to } \\
\text { wife) }\end{array}$ & $\begin{array}{l}\text { Husbands' job stress crossed } \\
\text { over the wives' } \\
\text { psychological well-being }\end{array}$ \\
\hline $\begin{array}{l}\text { Matthews, Del } \\
\text { Priore, Acitelli } \\
\text { \& Barnes- } \\
\text { Farrell (2006) }\end{array}$ & $\begin{array}{l}113 \text { dual- } \\
\text { earner couples }\end{array}$ & $\begin{array}{l}\text { Stressor } \rightarrow \\
\text { Strain }\end{array}$ & $\begin{array}{l}\text { Direct } \\
\text { Crossover }\end{array}$ & $\begin{array}{l}\text { Work } \rightarrow \\
\text { Home }\end{array}$ & $\begin{array}{l}\text { Work-to- } \\
\text { relationship } \\
\text { conflict on } \\
\text { outcomes } \\
\text { (relationship } \\
\text { tension, } \\
\text { health, } \\
\text { relationship } \\
\text { satisfaction) }\end{array}$ & $\begin{array}{l}\text { Unidirectional } \\
\text { (Wife to } \\
\text { husband) }\end{array}$ & $\begin{array}{l}\text { Direct crossover effect for } \\
\text { women's work-to- } \\
\text { relationship conflict on } \\
\text { men's reports of relationship } \\
\text { tension (and direct crossover } \\
\text { effect of men's work-to- } \\
\text { relationship conflict on } \\
\text { women's relationship } \\
\text { tension, although opposite } \\
\text { direction than was } \\
\text { hypothesized) }\end{array}$ \\
\hline Mitchell, & 157 couples & Stressor $\rightarrow$ & Direct & Home $\rightarrow$ & Negative & Unidirectional & Husbands' events and strain \\
\hline
\end{tabular}




\begin{tabular}{|c|c|c|c|c|c|c|c|}
\hline Citation & Sample & $\begin{array}{l}\text { Type of } \\
\text { Crossover }\end{array}$ & Mechanism & $\begin{array}{l}\text { Origin/ } \\
\text { Destination }\end{array}$ & Crossover of & Directionality & Findings \\
\hline $\begin{array}{l}\text { Cronkite \& } \\
\text { Moos (1983) }\end{array}$ & & $\begin{array}{l}\text { Strain; Strain } \\
\rightarrow \text { Strain }\end{array}$ & Crossover & Home & $\begin{array}{l}\text { events and } \\
\text { ongoing strain } \\
\text { on depression }\end{array}$ & $\begin{array}{l}\text { (Husband to } \\
\text { wife) }\end{array}$ & $\begin{array}{l}\text { were related to wives' } \\
\text { depression }\end{array}$ \\
\hline $\begin{array}{l}\text { Morrison \& } \\
\text { Clements } \\
(1997)\end{array}$ & $\begin{array}{l}82 \text { naval } \\
\text { couples }\end{array}$ & $\begin{array}{l}\text { Stressor } \rightarrow \\
\text { Strain; } \\
\text { Resource } \rightarrow \\
\text { Strain }\end{array}$ & $\begin{array}{l}\text { Direct } \\
\text { Crossover }\end{array}$ & $\begin{array}{l}\text { Work } \rightarrow \\
\text { Home }\end{array}$ & $\begin{array}{l}\text { Deployment } \\
\text { and the } \\
\text { mariners' job } \\
\text { characteristics } \\
\text { on well-being }\end{array}$ & $\begin{array}{l}\text { Unidirectional } \\
\text { (Mariner to } \\
\text { female } \\
\text { partner) }\end{array}$ & $\begin{array}{l}\text { A high level of male's role } \\
\text { ambiguity was associated } \\
\text { with the decreased well- } \\
\text { being of females. The return } \\
\text { of the male from deployment } \\
\text { was associated with elevated } \\
\text { mental health for the female. }\end{array}$ \\
\hline $\begin{array}{l}\text { Parasuraman, } \\
\text { Greenhaus \& } \\
\text { Granrose } \\
\text { (1992) }\end{array}$ & $\begin{array}{l}119 \text { dual- } \\
\text { career couples }\end{array}$ & $\begin{array}{l}\text { Stressor } \rightarrow \\
\text { Strain }\end{array}$ & $\begin{array}{l}\text { Direct } \\
\text { Crossover }\end{array}$ & $\begin{array}{l}\text { Home } \rightarrow \\
\text { Home }\end{array}$ & $\begin{array}{l}\text { Work and } \\
\text { family role } \\
\text { stressors and } \\
\text { work-family } \\
\text { conflict on } \\
\text { family } \\
\text { satisfaction }\end{array}$ & $\begin{array}{l}\text { Unidirectional } \\
\text { (Female to } \\
\text { male) }\end{array}$ & $\begin{array}{l}\text { Female's family role } \\
\text { stressors were negatively } \\
\text { related to spouse's family } \\
\text { satisfaction (no crossover } \\
\text { from male to female) }\end{array}$ \\
\hline Pavett (1986) & $\begin{array}{l}149 \text { CPAs and } \\
\text { their spouses }\end{array}$ & $\begin{array}{l}\text { Stressor } \rightarrow \\
\text { Strain }\end{array}$ & $\begin{array}{l}\text { Direct } \\
\text { Crossover }\end{array}$ & $\begin{array}{l}\text { Work } \rightarrow \\
\text { Home }\end{array}$ & $\begin{array}{l}\text { Stress on } \\
\text { physical and } \\
\text { psychological } \\
\text { symptoms }\end{array}$ & $\begin{array}{l}\text { Unidirectional } \\
\text { (CPA to } \\
\text { spouse) }\end{array}$ & $\begin{array}{l}\text { CPA's stress affected } \\
\text { spouses' physical and } \\
\text { psychological symptoms } \\
\text { burnout }\end{array}$ \\
\hline $\begin{array}{l}\text { Prince, } \\
\text { Manolis \& } \\
\text { Minetor } \\
(2007)\end{array}$ & $\begin{array}{l}86 \text { married } \\
\text { couples }\end{array}$ & $\begin{array}{l}\text { Resource } \rightarrow \\
\text { Resource }\end{array}$ & $\begin{array}{l}\text { Direct } \\
\text { Crossover }\end{array}$ & $\begin{array}{l}\text { Home } \rightarrow \\
\text { Home }\end{array}$ & $\begin{array}{l}\text { Life } \\
\text { satisfaction }\end{array}$ & $\begin{array}{l}\text { Bidirectional } \\
\text { (Spouse to } \\
\text { spouse) }\end{array}$ & $\begin{array}{l}\text { Crossover effect of life } \\
\text { satisfaction from one } \\
\text { spouse to another and vice } \\
\text { versa }\end{array}$ \\
\hline
\end{tabular}




\begin{tabular}{|c|c|c|c|c|c|c|c|}
\hline Citation & Sample & $\begin{array}{l}\text { Type of } \\
\text { Crossover }\end{array}$ & Mechanism & $\begin{array}{l}\text { Origin/ } \\
\text { Destination }\end{array}$ & Crossover of & Directionality & Findings \\
\hline $\begin{array}{l}\text { Riley \& } \\
\text { Eckenrode } \\
\text { (1986) }\end{array}$ & 314 women & $\begin{array}{l}\text { Stressor } \rightarrow \\
\text { Strain }\end{array}$ & $\begin{array}{l}\text { Direct } \\
\text { Crossover }\end{array}$ & $\begin{array}{l}\text { Home } \rightarrow \\
\text { Home }\end{array}$ & $\begin{array}{l}\text { Life events to } \\
\text { distress }\end{array}$ & $\begin{array}{l}\text { Unidirectional } \\
\text { (Male to } \\
\text { female) }\end{array}$ & $\begin{array}{l}\text { Women with lower levels of } \\
\text { personal resources were } \\
\text { distressed by the life events } \\
\text { of their significant others }\end{array}$ \\
\hline $\begin{array}{l}\text { Roberts \& } \\
\text { O'Keefe } \\
\text { (1981) }\end{array}$ & $\begin{array}{l}\text { Married } \\
\text { couples }(\mathrm{N}= \\
752)\end{array}$ & $\begin{array}{l}\text { Stressor } \rightarrow \\
\text { Strain }\end{array}$ & $\begin{array}{l}\text { Direct } \\
\text { Crossover }\end{array}$ & $\begin{array}{l}\text { Work } \rightarrow \\
\text { Home }\end{array}$ & $\begin{array}{l}\text { Employment } \\
\text { status on } \\
\text { depression }\end{array}$ & $\begin{array}{l}\text { Unidirectional } \\
\text { (Wife to } \\
\text { husband) }\end{array}$ & $\begin{array}{l}\text { The employment status of } \\
\text { the wife had no significant } \\
\text { effect on the husband's } \\
\text { depression }\end{array}$ \\
\hline $\begin{array}{l}\text { Rook, Dooley } \\
\text { \& Catalano } \\
\text { (1991) }\end{array}$ & $\begin{array}{l}1383 \text { married } \\
\text { women (with } \\
\text { a panel subset } \\
\text { of } 92 \text { ) }\end{array}$ & $\begin{array}{l}\text { Stressor } \rightarrow \\
\text { Strain }\end{array}$ & $\begin{array}{l}\text { Direct } \\
\text { Crossover }\end{array}$ & $\begin{array}{l}\text { Work } \rightarrow \\
\text { Home }\end{array}$ & $\begin{array}{l}\text { Job stressors } \\
\text { on emotional } \\
\text { health }\end{array}$ & $\begin{array}{l}\text { Unidirectional } \\
\text { (Husband to } \\
\text { wife) }\end{array}$ & $\begin{array}{l}\text { Husbands' stressors were } \\
\text { associated with significantly } \\
\text { elevated psychological } \\
\text { distress symptoms in wives }\end{array}$ \\
\hline $\begin{array}{l}\text { Rosenfield } \\
\text { (1980) }\end{array}$ & $\begin{array}{l}60 \text { married } \\
\text { couples }\end{array}$ & $\begin{array}{l}\text { Stressor } \rightarrow \\
\text { Strain }\end{array}$ & $\begin{array}{l}\text { Direct } \\
\text { Crossover }\end{array}$ & $\begin{array}{l}\text { Work } \rightarrow \\
\text { Home }\end{array}$ & $\begin{array}{l}\text { Employment } \\
\text { status on } \\
\text { depression }\end{array}$ & $\begin{array}{l}\text { Unidirectional } \\
\text { (Wife to } \\
\text { husband) }\end{array}$ & $\begin{array}{l}\text { Wife's employment was } \\
\text { positively related to } \\
\text { husband's distress }\end{array}$ \\
\hline $\begin{array}{l}\text { Rosenfield } \\
\text { (1992) }\end{array}$ & $\begin{array}{l}60 \text { married } \\
\text { couples }\end{array}$ & $\begin{array}{l}\text { Stressor } \rightarrow \\
\text { Strain }\end{array}$ & $\begin{array}{l}\text { Direct } \\
\text { Crossover }\end{array}$ & $\begin{array}{l}\text { Work } \rightarrow \\
\text { Home }\end{array}$ & $\begin{array}{l}\text { Employment } \\
\text { status on } \\
\text { psychological } \\
\text { well-being }\end{array}$ & $\begin{array}{l}\text { Unidirectional } \\
\text { (Wife to } \\
\text { husband) }\end{array}$ & $\begin{array}{l}\text { Wife's employment was } \\
\text { negatively related to } \\
\text { husband's mental health } \\
\text { only when wife's } \\
\text { employment decreased } \\
\text { husband's relative income } \\
\text { and increased share of } \\
\text { domestic labor }\end{array}$ \\
\hline $\begin{array}{l}\text { Staines, Pottic } \\
\text { \& Fudge }\end{array}$ & $\begin{array}{l}408 \text { husband- } \\
\text { housewife }\end{array}$ & $\begin{array}{l}\text { Stressor } \rightarrow \\
\text { Strain }\end{array}$ & $\begin{array}{l}\text { Direct } \\
\text { Crossover }\end{array}$ & $\begin{array}{l}\text { Work } \rightarrow \\
\text { Work; Work }\end{array}$ & $\begin{array}{l}\text { Employment } \\
\text { status on job }\end{array}$ & $\begin{array}{l}\text { Unidirectional } \\
\text { (Wife to }\end{array}$ & $\begin{array}{l}\text { Husbands of employed } \\
\text { wives had lower job and life }\end{array}$ \\
\hline
\end{tabular}




\begin{tabular}{|c|c|c|c|c|c|c|c|}
\hline Citation & Sample & $\begin{array}{l}\text { Type of } \\
\text { Crossover }\end{array}$ & Mechanism & $\begin{array}{l}\text { Origin/ } \\
\text { Destination }\end{array}$ & Crossover of & Directionality & Findings \\
\hline (1986) & $\begin{array}{l}\text { couples, } 208 \\
\text { husband- } \\
\text { working wife } \\
\text { couples }\end{array}$ & & & $\rightarrow$ Home & $\begin{array}{l}\text { and life } \\
\text { satisfaction }\end{array}$ & husband) & $\begin{array}{l}\text { satisfaction than husbands of } \\
\text { housewives }\end{array}$ \\
\hline $\begin{array}{l}\text { Takeuchi, Yun } \\
\& \text { Tesluk } \\
(2002)\end{array}$ & $\begin{array}{l}215 \text { Japanese } \\
\text { expatriates } \\
\text { and their } \\
\text { spouses }\end{array}$ & $\begin{array}{l}\text { Strain } \rightarrow \\
\text { Strain }\end{array}$ & $\begin{array}{l}\text { Direct } \\
\text { Crossover }\end{array}$ & $\begin{array}{l}\text { Home } \rightarrow \\
\text { Home }\end{array}$ & $\begin{array}{l}\text { Expatriate } \\
\text { cross-cultural } \\
\text { adjustment }\end{array}$ & $\begin{array}{l}\text { Bidirectional } \\
\text { (Expatriate to } \\
\text { spouse and } \\
\text { vice versa) }\end{array}$ & $\begin{array}{l}\text { Reciprocal crossover effects } \\
\text { between spouse and } \\
\text { expatriate general } \\
\text { adjustment }\end{array}$ \\
\hline $\begin{array}{l}\text { Van Emmerik } \\
\& \text { Peeters } \\
(2009)\end{array}$ & $\begin{array}{l}428 \text { Dutch } \\
\text { employees } \\
\text { working in } 49 \\
\text { teams }\end{array}$ & $\begin{array}{l}\text { Stressor } \rightarrow \\
\text { Stressor }\end{array}$ & $\begin{array}{l}\text { Direct } \\
\text { Crossover }\end{array}$ & $\begin{array}{l}\text { Work } \rightarrow \\
\text { Work; Home } \\
\rightarrow \text { Home }\end{array}$ & $\begin{array}{l}\text { Work-family } \\
\text { conflict }\end{array}$ & $\begin{array}{l}\text { Bidirectional } \\
\text { (Team to } \\
\text { individual and } \\
\text { vice versa) }\end{array}$ & $\begin{array}{l}\text { Team-level work-to-family } \\
\text { conflict had a crossover } \\
\text { effect on individual-level } \\
\text { work-to-family conflict and } \\
\text { team-level family-to-work } \\
\text { conflict had a crossover } \\
\text { effect on individual-level } \\
\text { family-to-work conflict }\end{array}$ \\
\hline $\begin{array}{l}\text { Vinokur, Price } \\
\text { \& Caplan } \\
\text { (1996) }\end{array}$ & $\begin{array}{l}815 \text { job } \\
\text { seekers and } \\
\text { their spouses }\end{array}$ & $\begin{array}{l}\text { Stressor } \rightarrow \\
\text { Strain }\end{array}$ & $\begin{array}{l}\text { Common } \\
\text { Stressors; } \\
\text { Indirect } \\
\text { Crossover }\end{array}$ & $\begin{array}{l}\text { Home } \rightarrow \\
\text { Home }\end{array}$ & $\begin{array}{l}\text { Financial } \\
\text { strain and } \\
\text { social } \\
\text { undermining } \\
\text { on depression }\end{array}$ & $\begin{array}{l}\text { Unidirectional } \\
\text { (Job-seeker to } \\
\text { spouse) }\end{array}$ & $\begin{array}{l}\text { Partner's depression is } \\
\text { affected by common } \\
\text { stressors and undermining } \\
\text { transactions }\end{array}$ \\
\hline $\begin{array}{l}\text { Westman \& } \\
\text { Etzion (1995) }\end{array}$ & $\begin{array}{l}101 \text { Israeli } \\
\text { male military } \\
\text { officers and } \\
\text { their working } \\
\text { wives }\end{array}$ & $\begin{array}{l}\text { Strain } \rightarrow \\
\text { Strain; } \\
\text { Stressor } \rightarrow \\
\text { Strain; } \\
\text { Stressor } \rightarrow\end{array}$ & $\begin{array}{l}\text { Direct } \\
\text { Crossover }\end{array}$ & $\begin{array}{l}\text { Home } \rightarrow \\
\text { Home }\end{array}$ & $\begin{array}{l}\text { Burnout; } \\
\text { Sense of } \\
\text { control on } \\
\text { burnout; sense } \\
\text { of control }\end{array}$ & $\begin{array}{l}\text { Bidirectional } \\
\text { (Spouse to } \\
\text { spouse); } \\
\text { Unidirectional } \\
\text { (Officer to }\end{array}$ & $\begin{array}{l}\text { Bidirectional crossover of } \\
\text { burnout; crossover of the } \\
\text { male partner's sense of } \\
\text { control on the female } \\
\text { partner's burnout levels; }\end{array}$ \\
\hline
\end{tabular}




\begin{tabular}{|c|c|c|c|c|c|c|c|}
\hline Citation & Sample & $\begin{array}{l}\text { Type of } \\
\text { Crossover }\end{array}$ & Mechanism & $\begin{array}{l}\text { Origin/ } \\
\text { Destination }\end{array}$ & Crossover of & Directionality & Findings \\
\hline & & Stressor & & & & $\begin{array}{l}\text { wife); } \\
\text { Bidirectional }\end{array}$ & $\begin{array}{l}\text { bidirectional crossover of } \\
\text { sense of control }\end{array}$ \\
\hline $\begin{array}{l}\text { Westman \& } \\
\text { Etzion (1999) }\end{array}$ & $\begin{array}{l}83 \text { principals } \\
\text { and } 283 \\
\text { teachers in the } \\
\text { same schools } \\
\text { in Israel }\end{array}$ & $\begin{array}{l}\text { Strain } \rightarrow \\
\text { Strain }\end{array}$ & $\begin{array}{l}\text { Direct } \\
\text { Crossover }\end{array}$ & $\begin{array}{l}\text { Work } \rightarrow \\
\text { Work }\end{array}$ & $\begin{array}{l}\text { Tension (but } \\
\text { not burnout) }\end{array}$ & $\begin{array}{l}\text { Bidirectional } \\
\text { (Principals to } \\
\text { teachers and } \\
\text { vice versa) }\end{array}$ & $\begin{array}{l}\text { Significant crossover of job- } \\
\text { induced tension between } \\
\text { principals and teachers and } \\
\text { vice versa }\end{array}$ \\
\hline $\begin{array}{l}\text { Westman \& } \\
\text { Etzion (2005) }\end{array}$ & $\begin{array}{l}220 \text { women in } \\
\text { the Air Force } \\
\text { and their } \\
\text { working } \\
\text { spouses }\end{array}$ & $\begin{array}{l}\text { Stressor } \rightarrow \\
\text { Stressor }\end{array}$ & $\begin{array}{l}\text { Direct } \\
\text { Crossover }\end{array}$ & $\begin{array}{l}\text { Work } \rightarrow \\
\text { Work; Family } \\
\rightarrow \text { Family }\end{array}$ & $\begin{array}{l}\text { Work-family } \\
\text { conflict }\end{array}$ & $\begin{array}{l}\text { Bidirectional } \\
\text { (Spouse to } \\
\text { spouse) }\end{array}$ & $\begin{array}{l}\text { Bidirectional crossover of } \\
\text { work-to-family conflict and } \\
\text { family-to-work conflict }\end{array}$ \\
\hline $\begin{array}{l}\text { Westman, } \\
\text { Etzion \& } \\
\text { Chen (2009) }\end{array}$ & $\begin{array}{l}275 \text { business } \\
\text { travelers and } \\
\text { their working } \\
\text { spouses }\end{array}$ & $\begin{array}{l}\text { Resource } \rightarrow \\
\text { Resource }\end{array}$ & $\begin{array}{l}\text { Direct } \\
\text { Crossover }\end{array}$ & $\begin{array}{l}\text { Work } \rightarrow \\
\text { Work }\end{array}$ & $\begin{array}{l}\text { Vigor } \\
\text { (component of } \\
\text { engagement) }\end{array}$ & $\begin{array}{l}\text { Unidirectional } \\
\text { (Traveler to } \\
\text { spouse) }\end{array}$ & $\begin{array}{l}\text { Traveler's vigor crossed } \\
\text { over to spouse's vigor }\end{array}$ \\
\hline $\begin{array}{l}\text { Westman, } \\
\text { Etzion \& } \\
\text { Danon (2001) }\end{array}$ & $\begin{array}{l}98 \text { Israeli } \\
\text { married } \\
\text { couples }\end{array}$ & $\begin{array}{l}\text { Strain } \rightarrow \\
\text { Strain }\end{array}$ & $\begin{array}{l}\text { Direct } \\
\text { Crossover; } \\
\text { Indirect } \\
\text { Crossover }\end{array}$ & $\begin{array}{l}\text { Work } \rightarrow \\
\text { Work }\end{array}$ & Burnout & $\begin{array}{l}\text { Unidirectional } \\
\text { (Husband to } \\
\text { wife) }\end{array}$ & $\begin{array}{l}\text { Direct crossover of burnout } \\
\text { from husbands to wives, } \\
\text { supported the impact of } \\
\text { burnout on undermining } \\
\text { behavior }\end{array}$ \\
\hline $\begin{array}{l}\text { Westman, } \\
\text { Keinan, } \\
\text { Roziner \& } \\
\text { Benyamini }\end{array}$ & $\begin{array}{l}\text { 2,108 Russian } \\
\text { couples }\end{array}$ & $\begin{array}{l}\text { Strain } \rightarrow \\
\text { Strain, } \\
\text { Stressor } \rightarrow \\
\text { Strain }\end{array}$ & $\begin{array}{l}\text { Direct } \\
\text { Crossover, } \\
\text { Indirect } \\
\text { Crossover, }\end{array}$ & $\begin{array}{l}\text { Home } \rightarrow \\
\text { Home }\end{array}$ & $\begin{array}{l}\text { Perceived } \\
\text { health, } \\
\text { common } \\
\text { stressor of }\end{array}$ & $\begin{array}{l}\text { Bidirectional } \\
\text { (Spouse to } \\
\text { spouse) }\end{array}$ & $\begin{array}{l}\text { Perceived health crossed } \\
\text { over directly between } \\
\text { spouses, common stressors } \\
\text { affected perceived health }\end{array}$ \\
\hline
\end{tabular}




\begin{tabular}{|c|c|c|c|c|c|c|c|}
\hline Citation & Sample & $\begin{array}{l}\text { Type of } \\
\text { Crossover }\end{array}$ & Mechanism & $\begin{array}{l}\text { Origin/ } \\
\text { Destination }\end{array}$ & Crossover of & Directionality & Findings \\
\hline (2008) & & & $\begin{array}{l}\text { Common } \\
\text { Stressors }\end{array}$ & & $\begin{array}{l}\text { economic } \\
\text { hardship, } \\
\text { undermining }\end{array}$ & & $\begin{array}{l}\text { directly and indirectly via } \\
\text { undermining }\end{array}$ \\
\hline $\begin{array}{l}\text { Westman \& } \\
\text { Vinokur } \\
(1998)\end{array}$ & $\begin{array}{l}354 \text { male } \\
\text { veterans and } \\
\text { their partners }\end{array}$ & $\begin{array}{l}\text { Strain } \rightarrow \\
\text { Strain; } \\
\text { Stressor } \rightarrow \\
\text { Strain }\end{array}$ & $\begin{array}{l}\text { Direct } \\
\text { Crossover; } \\
\text { Common } \\
\text { Stressors; } \\
\text { Indirect } \\
\text { Crossover }\end{array}$ & $\begin{array}{l}\text { Home } \rightarrow \\
\text { Home }\end{array}$ & Depression & $\begin{array}{l}\text { Bidirectional } \\
\text { (Partner to } \\
\text { partner) }\end{array}$ & $\begin{array}{l}\text { Direct crossover of } \\
\text { depression; life events } \\
\text { served a common stressor; } \\
\text { social undermining mediated } \\
\text { the process }\end{array}$ \\
\hline $\begin{array}{l}\text { Westman, } \\
\text { Vinokur, } \\
\text { Hamilton \& } \\
\text { Roziner } \\
(2004)\end{array}$ & $\begin{array}{l}1609 \text { Russian } \\
\text { army officers } \\
\text { and wives }\end{array}$ & $\begin{array}{l}\text { Strain } \rightarrow \\
\text { Strain }\end{array}$ & $\begin{array}{l}\text { Direct } \\
\text { Crossover; } \\
\text { Indirect } \\
\text { Crossover }\end{array}$ & $\begin{array}{l}\text { Home } \rightarrow \\
\text { Home }\end{array}$ & $\begin{array}{l}\text { Marital } \\
\text { dissatisfaction }\end{array}$ & $\begin{array}{l}\text { Unidirectional } \\
\text { (Husband to } \\
\text { wife) }\end{array}$ & $\begin{array}{l}\text { Found direct crossover of } \\
\text { marital dissatisfaction from } \\
\text { husband to wife; Found an } \\
\text { indirect crossover to be } \\
\text { mediated by the impact of } \\
\text { the wife's social } \\
\text { undermining behavior on her } \\
\text { husband }\end{array}$ \\
\hline
\end{tabular}




\section{Appendix B}

\section{Study Measures}

\section{$\underline{\text { Job Content Questionnaire }}$}

\section{Skill Discretion Subscale}

\begin{tabular}{|l|l|l|l|l|l|}
\hline In my job... & $\begin{array}{c}\text { Strongly } \\
\text { Disagree }\end{array}$ & Disagree & Neutral & Agree & $\begin{array}{c}\text { Strongly } \\
\text { Agree }\end{array}$ \\
\hline My job requires that I learn new things & & & & & \\
\hline $\begin{array}{l}\text { I have an opportunity to develop my } \\
\text { own special abilities }\end{array}$ & & & & & \\
\hline My job requires a high level of skill & & & & & \\
\hline $\begin{array}{l}\text { I get to do a variety of things on my } \\
\text { job }\end{array}$ & & & & & \\
\hline $\begin{array}{l}\text { My job requires a lot of repetitive } \\
\text { work }\end{array}$ & & & & & \\
\hline My job requires me to be creative & & & & & \\
\hline
\end{tabular}

Decision Authority Subscale

\begin{tabular}{|l|l|l|l|l|l|}
\hline In my job... & $\begin{array}{c}\text { Strongly } \\
\text { Disagree }\end{array}$ & Disagree & Neutral & Agree & $\begin{array}{c}\text { Strongly } \\
\text { Agree }\end{array}$ \\
\hline $\begin{array}{l}\text { My job allows me to make a lot of } \\
\text { decisions on my own. }\end{array}$ & & & & & \\
\hline $\begin{array}{l}\text { On my job, I am given a lot of } \\
\text { freedom to decide how I do my work. }\end{array}$ & & & & & \\
\hline $\begin{array}{l}\text { I have a lot of say about what happens } \\
\text { on my job. }\end{array}$ & & & & & \\
\hline
\end{tabular}

Psychological Demands Subscale

\begin{tabular}{|l|l|l|l|l|l|}
\hline In my job... & $\begin{array}{c}\text { Strongly } \\
\text { Disagree }\end{array}$ & Disagree & Neutral & Agree & $\begin{array}{c}\text { Strongly } \\
\text { Agree }\end{array}$ \\
\hline $\begin{array}{l}\text { I am not asked to do an excessive } \\
\text { amount of work. }\end{array}$ & & & & & \\
\hline $\begin{array}{l}\text { I am free from conflicting demands } \\
\text { others make. }\end{array}$ & & & & & \\
\hline $\begin{array}{l}\text { I have enough time to get the job } \\
\text { done. }\end{array}$ & & & & & \\
\hline My job requires working very fast. & & & & & \\
\hline My job requires working very hard. & & & & & \\
\hline
\end{tabular}




\section{Income Adequacy}

Which of the following statements describes your ability to get along on your income?

We can't make ends meet

We have just enough, no more

We have enough, with a little extra, sometimes

We always have money left over

\section{SF-12 Health and Well-Being Survey}

I would like to now ask you about your general health, daily activities, and social interactions in the last month. I'm going to show you a card and ask you to point to the answer that most closely fits your experience. We're going to go through this quickly.

1. In general, would you say your health is:

\begin{tabular}{|lllll|}
\hline Excellent & Very good & Good & Fair & Poor \\
$\square$ & $\nabla$ & $\square$ & & \\
\hline & $\square$ & $\square$ & $\square$ & $\square$
\end{tabular}

2. The following questions are about activities you might do during a typical day. Does your health now limit you in these activities? If so, how much?

\begin{tabular}{|ccc|}
\hline Yes, & Yes, & No, not \\
limited & limited & limited \\
a lot & a little & at all \\
\hline
\end{tabular}

a Moderate activities, such as moving a table,

pushing a vacuum cleaner, bowling, or

playing golf

b Climbing several flights of stairs

[prompt: more than 3 flights of stairs] 
3. During the past 4 weeks, how much of the time have you had any of the following problems with your work or other regular daily activities as a result of your physical health?

$\begin{array}{|ccccc|}\begin{array}{c}\text { All of } \\ \text { the }\end{array} & \begin{array}{c}\text { Most of } \\ \text { the }\end{array} & \begin{array}{c}\text { Some } \\ \text { of the }\end{array} & \begin{array}{c}\text { A little } \\ \text { of the }\end{array} & \begin{array}{c}\text { None of } \\ \text { the }\end{array} \\ \text { time } & \text { time } & \text { time } & \text { time } & \text { time }\end{array}$

a Accomplished less than you would like

b Were limited in the kind of work or

other activities

4. During the past 4 weeks, how much of the time have you had any of the following problems with your work or other regular daily activities as a result of any emotional problems (such as feeling depressed or anxious)?

\begin{tabular}{ccccc|}
$\begin{array}{c}\text { All of } \\
\text { the }\end{array}$ & Most of & Some & A little & None of \\
the & of the & of the & the \\
time & time & time & time & time
\end{tabular}

a Accomplished less than you would like

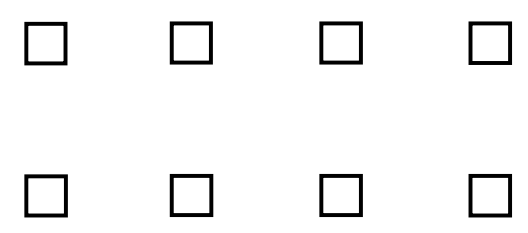
carefully than usual 
5. During the past 4 weeks, how much did pain interfere with your normal work (including both work outside the home and housework)?

\begin{tabular}{|lcccc|}
\hline Not at all & A little bit & Moderately & Quite a bit & Extremely \\
$\square$ & $\square$ & $\square$ & $\square$ & $\square$
\end{tabular}

6. These questions are about how you feel and how things have been with you during the past 4 weeks. For each question, please give the one answer that comes closest to the way you have been feeling. How much of the time during the past 4 weeks...

\begin{tabular}{|c|c|c|c|}
\hline $\begin{array}{c}\text { All of } \\
\text { the time }\end{array}$ & $\begin{array}{l}\text { Most of } \\
\text { the time }\end{array}$ & $\begin{array}{l}\text { Some of } \\
\text { the time }\end{array}$ & $\begin{array}{l}\text { A little } \\
\text { of the } \\
\text { time }\end{array}$ \\
\hline
\end{tabular}

a Have you felt calm and peaceful?

b Did you have a lot of energy

c Have you felt downhearted and

depressed?

7. During the past 4 weeks, how much of the time has your physical health or emotional problems interfered with your social activities (like visiting friends, relatives, etc.)?

\begin{tabular}{ccccc|}
$\begin{array}{c}\text { All of the } \\
\text { time }\end{array}$ & $\begin{array}{c}\text { Most of the } \\
\text { time }\end{array}$ & $\begin{array}{c}\text { Some of the } \\
\text { time }\end{array}$ & $\begin{array}{c}\text { A little of the of the } \\
\text { time }\end{array}$ \\
$\square$ & & time
\end{tabular}

